JANTJE FreudenthaL

FOLLOW-UP AND DYNAMICAL ANALYSIS OF KEPLER TARGETS WITH TRANSIT TIMING VARIATIONS

Dissertation 



\title{
Follow-up and dynamical analysis of Kepler targets with transit timing variations
}

\author{
Dissertation \\ for the award of the degree \\ "Doctor rerum naturalium" (Dr.rer.nat.) \\ of the Georg-August-Universität Göttingen \\ within the doctoral program PROPHYS \\ of the Georg-August University School of Science (GAUSS) \\ submitted by \\ Jantje Freudenthal \\ from Achim
}

Göttingen, 2019 


\section{Thesis Commitee}

Prof. Dr. Stefan Dreizler

Sonnenphysik und Stellare Astrophysik, Institut für Astrophysik, Georg-August-Universität Göttingen, Germany

Dr. Carolina von Essen

Stellar Astrophysics Centre, Department of Physics and Astronomy, Aarhus University, Denmark

Dr. Frederic V. Hessman

Sonnenphysik und Stellare Astrophysik, Institut für Astrophysik, Georg-August-Universität Göttingen, Germany

Members of the Examination Board

Reviewer: Prof. Dr. Stefan Dreizler

Sonnenphysik und Stellare Astrophysik, Institut für Astrophysik, Georg-August-Universität Göttingen, Germany

Second Reviewer: Prof. Dr. Laurent Gizon

Department Solar and Stellar Interiors, Max-Planck-Institut für Sonnensystemforschung, Göttingen, Germany

Further members of the Examination Board:

Prof. Dr. Wolfram Kollatschny

Extragalaktische Astrophysik und Kosmologie, Institut für Astrophysik, Georg-AugustUniversität Göttingen, Germany

Prof. Dr. Jens Niemeyer

Extragalaktische Astrophysik und Kosmologie, Institut für Astrophysik, Georg-AugustUniversität Göttingen, Germany

Prof. Dr. Karl-Henning Rehren

Relativistische Quantenfeldtheorie, Institut für Theoretische Physik, Georg-AugustUniversität Göttingen, Germany

Prof. Dr. Ansgar Reiners

Sonnenphysik und Stellare Astrophysik, Institut für Astrophysik, Georg-August-Universität Göttingen, Germany

Date of the oral examination: 01 November 2019 




\section{Abstract}

The exact characterisation of planets, their host stars and the structure of their systems is an essential part of exoplanet research. This helps to understand the formation and evolution of planetary systems. Planets detected with the transiting method that show transit timing variations (TTVs) are particularly suitable for a detailed characterisation of their system. TTVs result from dynamical interactions between the system objects. Hence, from TTVs the orbital configuration and the planetary masses are determinable. Together with the radius defined by the transits, the planetary density is calculable, which helps to understand the planetary nature. This thesis is dedicated to follow-up observations and the dynamical modelling of TTV planetary systems in order to enable a refined system characterisation. Two transiting planetary systems discovered with the Kepler telescope and containing TTV planets are targets of this characterisation. To extend the observation baseline with the aim of capturing the full dynamic cycle of the TTV curves, ground-based follow-up observations of the planets transits were performed and processed within the framework of the KOINet (Kepler Object of Interest Network). To enable a comprehensive and self-consistent analysis of the systems a photodynamical model was developed for the entire photometric light curve. The photodynamical model performs a numerical integration of the entire system over the time span of observations taking into account the dynamical interactions between all objects and calculates transit light curves from the output. Kepler-9 is the first of the two systems which was subject to KOINet follow-up observations and the photodynamical analysis. The two planets b and c show anticorrelated, sinusoidal-like TTV curves. The photodynamical analysis of the system results in precise planet parameter determinations of the order of $\sim 1 \%$, which makes them the planets with the best determined densities in the Neptune mass regime. In addition, the analysis predicts that the transits of Kepler-9c will disappear by 2050 due to orbital precession as a result of the strong interaction between the planets. Correspondingly, planet b will migrate towards the lower latitudes of the star. In the next 30 years the latitudes of the star will be scanned by the transits of the planets, where planet $\mathrm{b}$ will move towards possible spot regions and planet $\mathrm{c}$ will explore the limb of the star before disappearing. The second analysed system is Kepler-82 with the TTV-showing planets b and c. Here, the TTVs are not anti-correlated and the curve of planet c exhibits jumps every three consecutive transits, this feature is called chopping signal. The chopping signal is not induced by planet $\mathrm{b}$ but originated by a third outer component. With only Kepler data, two possibly system configurations are found, where an outer planet is near a 3:2 or 3:1 period ratio to planet c. The 
adding of KOINet follow-up observations leads to a unique solution resulting in the detection of a new non-transiting planet in the system, Kepler-82f, orbiting the star near a 3:2 commensurability to planet c. Both systems are examples of how planets in transiting systems can be missed in the light curves, since the dynamical interaction between planets can cause small deviations from co-planarity. Kepler-9c would have been missed if the Kepler mission would had been launched 40 years later and the Kepler-82 system could have shown a completely different combination of transiting planets if it had been observed at another time. The follow-up observations of the systems and their analysis with the self-consistent photodynamical model developed here enabled the precise parameter determination and system characterisation, which led to the prediction of the disappearance of the transits of Kepler-9c and to the discovery of the planet Kepler-82f. 


\section{Zusammenfassung}

Die genaue Charakterisierung von Planeten, ihren Heimatsternen und dem Aufbau ihrer Systeme ist ein wesentlicher Teil der Exoplanetenforschung. Dies hilft dabei die Entstehungs- und Entwicklungsgeschichte von Planetensystemen zu verstehen. Mit der Transitmethode detektierte Planeten, die Transitzeitvariationen (engl. transit timing variations, TTVs) aufweisen, eignen sich besonders für eine detaillierte Charakterisierung ihres Systems. TTVs sind das Ergebnis dynamischer Interaktionen zwischen den Systemobjekten. Aus TTVs sind somit die Orbitalkonfiguration des Systems und die Planetenmassen bestimmbar. Zusammen mit dem Radius, definiert durch den Transit, ist die planetarische Dichte bestimmbar, was hilft, die Natur des Planeten zu verstehen. Diese Arbeit widmet sich Folgebeobachtungen und der dynamischen Modellierung von TTV-Planetensystemen, um eine verfeinerte Systemcharakterisierung zu ermöglichen. Zwei Transit-Planetensysteme, die mit dem Kepler -Teleskop entdeckt wurden und TTV-Planeten enthalten, sind Ziele dieser Charakterisierung. Zur Erweiterung der Beobachtungsbasislinie mit dem Ziel, den vollen dynamischen Zyklus der TTV-Kurven zu erfassen, wurden im Rahmen des KOINet (Kepler Object of Interest Network) bodengebundene Folgebeobachtungen der Planetentransite durchgeführt und verarbeitet. Um eine umfassende und selbstkonsistente Analyse der Systeme zu ermöglichen, wurde ein photodynamisches Modell für die gesamte photometrische Lichtkurve erstellt. Das photodynamische Modell führt eine numerische Integration des Gesamtsystems über die Gesamtzeit aller Beobachtungen unter Berücksichtigung der dynamischen Wechselwirkungen zwischen allen Objekten durch und berechnet aus der Ausgabe Transitlichtkurven. Kepler-9 ist das erste der beiden Systeme, das den KOINet Folgebeobachtungen und der photodynamischen Analyse unterzogen wurde. Die beiden Planeten b und c zeigen antikorrelierte, sinusförmige TTV-Kurven. Die photodynamische Analyse des Systems führt zu präzisen Planetenparameterbestimmungen in der Größenordnung von $\sim 1 \%$, was sie zu den Planeten mit den am besten bestimmten Dichten im Neptun-Massenregime macht. Darüber hinaus prognostiziert die Analyse, dass die Transite von Kepler-9c um 2050 aufgrund der orbitalen Präzession als Folge der starken Interaktion der Planeten verschwinden werden. Dementsprechend wandert Planet b in Richtung der geringeren Breitengrade des Sterns. In den nächsten 30 Jahren werden die Breitengrade des Sterns durch die Transite der Planeten gescannt, wobei sich Planet b in Richtung möglicher Sternfleckregionen bewegt und Planet c den Rand des Sterns erforscht, bevor er verschwindet. Das zweite analysierte System ist Kepler-82 mit den TTVzeigenden Planeten b und c. In diesem System sind die TTVs nicht antikorreliert 
und die Kurve des Planeten c zeigt Sprünge alle drei aufeinanderfolgenden Transits. Dieses Signal wird nicht vom Planeten b induziert, sondern von einer dritten äußeren Komponente erzeugt. Mit nur den Kepler Daten werden zwei mögliche Systemkonfigurationen gefunden, bei denen ein äußerer Planet in der Nähe eines 3:2 oder 3:1 Periodenverhältnisses zum Planeten c liegt. Das Hinzufügen von KOINet-Follow-upBeobachtungen führt zu einer eindeutigen Lösung, die zur Entdeckung eines neuen, keine Transits aufweisenden Planeten im System führt, Kepler-82f, der den Stern in der Nähe eines 3:2 Periodenverhältnisses zum Planeten c umkreist. Beide Systeme sind Beispiele dafür, wie Planeten in Transitsystemen in den Lichtkurven übersehen werden können, da durch die dynamische Interaktion zwischen den Planeten geringe Abweichungen von der Koplanarität hervorgerufen werden können. Kepler-9c wäre übersehen worden, wenn die Kepler Mission 40 Jahre später gestartet worden wäre und das Kepler-82-System hätte eine völlig andere Kombination von Transitplaneten zeigen können, wenn man es zu einem anderen Zeitpunkt beobachtet hätte. Durch die Nachbeobachtungen der Systeme und die Analyse mit dem hier entwickelten selbstkonsistenten photodynamischen Modells wurde die präzise Parameterbestimmung und Systemcharakterisierung ermöglicht, die zu der Vorhersage des Verschwindens der Transite von Kepler-9c und der Entdeckung des Planeten Kepler-82f führte. 


\section{Contents}

1 Introduction 1

1.1 Exoplanet detection methods . . . . . . . . . . . . . . . . . . . . 1

1.1.1 Radial velocity method . . . . . . . . . . . . . . . . . . 3

1.1.2 Transit method . . . . . . . . . . . . . . . . . 5

1.2 Transit timing variations . . . . . . . . . . . . . . . . . . . . . 6

1.2 .1 Multi-planet systems . . . . . . . . . . . . . . . . . . . 8

1.2 .2 Mean-motion resonances . . . . . . . . . . . . . . . . 11

1.2 .3 Different types of TTVs . . . . . . . . . . . . . . . . 13

1.3 The KOINet . . . . . . . . . . . . . . . . . . . . . . . . . . . . . . 15

1.3 .1 Light curve extraction . . . . . . . . . . . . . . . . . . . . 17

1.3 .2 Detrending and transit modelling . . . . . . . . . . . . . . . 18

1.4 Photodynamical modelling . . . . . . . . . . . . . . . . . . 21

1.4 .1 The algorithm . . . . . . . . . . . . . . . . . . . . . . . . . . . . . . . . 22

1.4.2 $\quad$ Data-algorithm correspondence . . . . . . . . . . . . . . . 22

1.4 .3 Model parameters . . . . . . . . . . . . . . . . . . . 23

1.5 This thesis . . . . . . . . . . . . . . . . . . . . . . 24

$\begin{array}{lll}2 & \text { Kepler-9 } & 27\end{array}$

2.1 Paper: Kepler Object of Interest Network II. Photodynamical modelling of Kepler-9 over 8 years of transit observations . . . . . . . . . 27

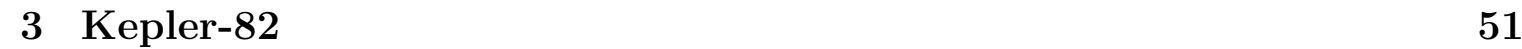

3.1 Paper: Kepler Object of Interest Network III. Kepler-82f: A new nontransiting $21 M_{\oplus}$ planet from photodynamical modelling . . . . . . . . 51

\begin{tabular}{lll}
\hline & Summary, conclusion and outlook & 71
\end{tabular}

4.1 The disappearing transits of Kepler-9c . . . . . . . . . . . . . . . . 71

4.2 A non-transiting planet orbiting Kepler-82 . . . . . . . . . . . . . 73

4.3 Multi-planet systems _. . . . . . . . . . . . . . . . . . . . . 74

4.4 Correlated noise . . . . . . . . . . . . . . . . . . . . . . . . . . 75

4.5 Future Observations . . . . . . . . . . . . . . . . . . . . . . . . . . . 79

\begin{tabular}{lr}
\hline Bibliography & 81
\end{tabular} 


\section{Chapter 1}

\section{Introduction}

Looking into the night sky (in a rural place), we can see thousands of sparkling stars. The question about other worlds like our Earth, about life existing elsewhere in the sheer endless universe is not that far-fetched and has occupied mankind probably already almost since its origin (for more than 2000 years from writings). Only 25 years ago, science took a big step forward to answer this question by discovering the first planet orbiting a star other than our Sun (Mayor \& Queloz, 1995). Since then, thousands of exoplanets have been detected and there are many ongoing and future missions dedicated to finding many more of them. The zoo of discovered exoplanets is enriched by outstanding detections such as exoplanets in the close proximity of the Sun: an Earth-mass planet orbiting the closest star, Proxima centauri AngladaEscudé et al., 2016); two Earth-like planets orbiting Teegarden's Star in the habitable zone (Zechmeister et al., 2019); and seven Earth-like planets in the Trappist-1 system, four of which may be habitable (Gillon et al., 2017). The Planetary Habitability Laboratory ${ }^{1}$ reports a total of 55 potentially habitable exoplanets (2019 September 15) that could host life similar to that on Earth.

There is probably still a long way to go before we discover extraterrestrial life and many more aspects are important than the detection of Earth-like planets in the habitable zone of their home stars. In order to gain a greater understanding of the universe and the formation of galaxies, stars and planetary systems, the domain of exoplanet science comprises a broad field of interest. The discovery and accurate characterisation of many different exoplanets and planetary systems and their dynamics are important milestones along this path.

\subsection{Exoplanet detection methods}

There are many methods for detecting exoplanets. In Figure 1.1 Micheal Perryman has outlined the different methods and their current and prospected lower planet mass detectability limits (similar to Figure 1.1 in Perryman, 2018). The methods are categorised into three different fields: dynamical, microlensing, and photometry. In the following, a summary of the detection techniques in these categories is given. An

\footnotetext{
${ }^{1}$ http://phl.upr.edu/projects/habitable-exoplanets-catalog
} 


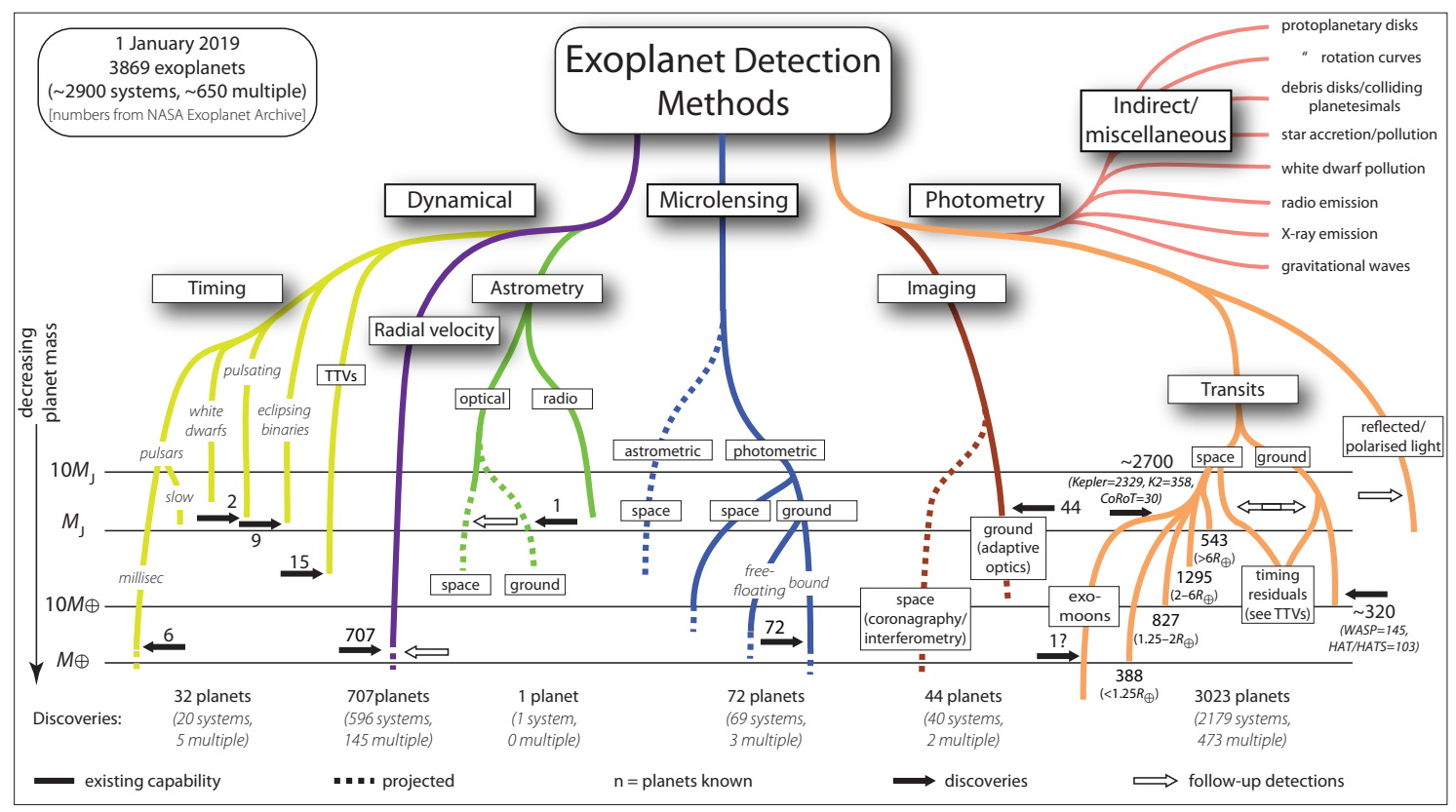

Figure 1.1: The various planet detection methods and there capability in the mass regime, given on logarithmic scale. The solid lines indicate the current capability of the methods and the dashed line the future prospective. Numbers give the number of known planets detected with the methods. The mass regime capabilities of the indirect or miscellaneous methods are not known so far, so the axis is not applying for them. Diagram by Michael Perryman with data from 2019 January 1, similar to Figure 1.1 in Perryman (2018).

interesting aspect is the total number of planets detected by the respective approach: the cumulative number of detections per year and per planet detection method is plotted in Figure 1.2.

Dynamical methods are based on the gravitational effect of an exoplanet on its host star or other measurable objects in the system. Planet detections via radial velocity measurements is one of the two most successful detection methods (see Figure 1.2) and located in the field of dynamical methods. This observation technique is based on stellar radial velocity changes in the line-of-sight of the observer due to an orbiting exoplanet; the details of this method will be discussed later this section. In astrometric observations the change of the position of the star due to an exoplanet is measured. The timing method contains measurements of deviations from periodic events. These deviations could be a light-travel time effect if the star is the source of the periodic events and its position is changed due to the exoplanet. The periodicity hereby can be given by pulsars, pulsating stars, or eclipsing binaries for example. Furthermore, the later discussed transit events can be time shifted by various reasons due to further planets in the system. These so-called transit timing variations are discussed in detail in section 1.2.

For the microlensing method the gravitational lensing effect is utilised. A foreground star that moves in front of a background source magnifies the light coming 


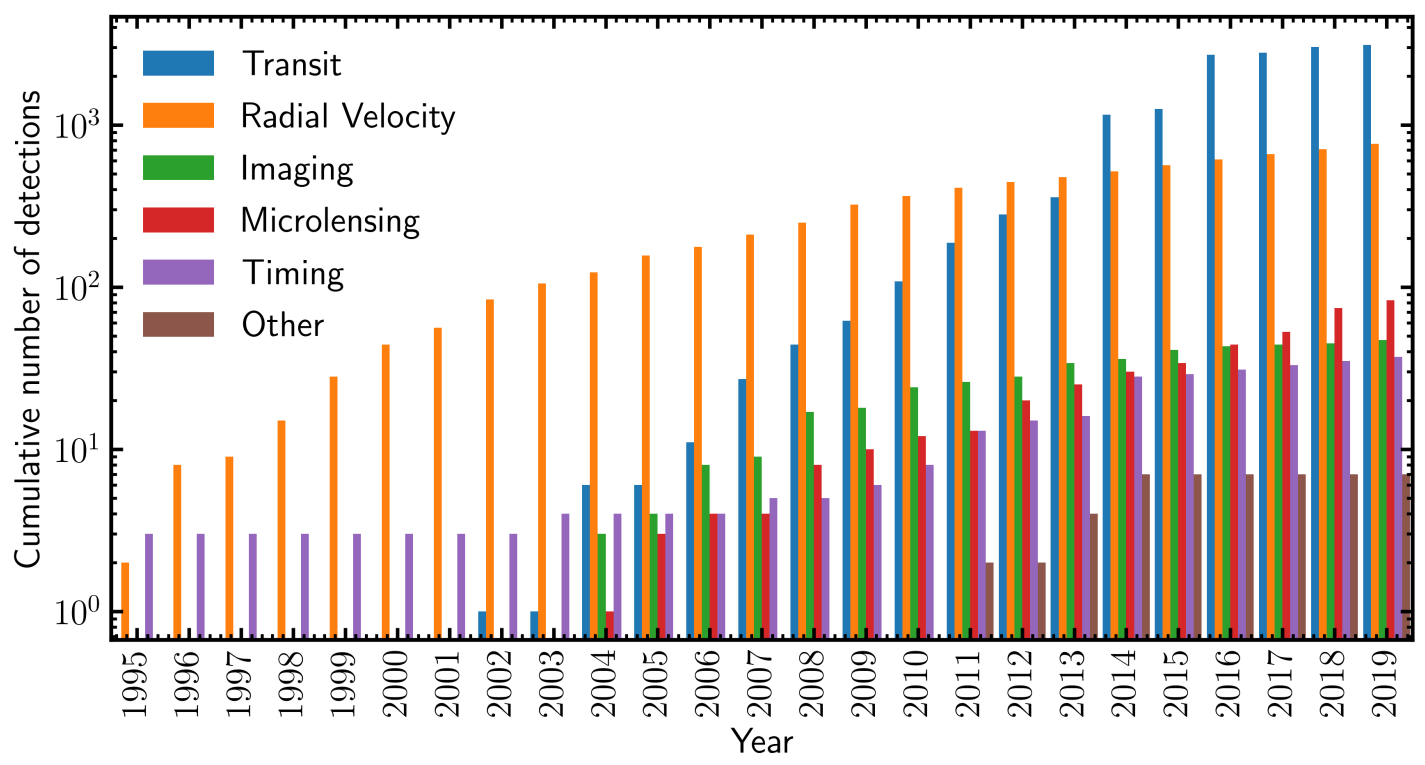

Figure 1.2: The cumulative number of detections per year and planet detection method. Listed techniques are the transit method, radial velocity measurements, direct imaging, planets detected by microlensing, and timing effects. All other methods are combined in "others". The numbers are from the NASA Exoplanet Archive, 2019 September 15.

from this source. A planet orbiting the lens star acts as a second lens, measurable as a secondary signal in the light curve of the event. Such events are unique and not reproducible. Therefore, follow-up observations of the detected exoplanet can only be performed with another planet detection method.

With photometric measurements three different planet detection methods are applicable. When a sufficient spatial resolution is given, large planets in a large orbit around a masked star can be directly imaged. Furthermore, the reflected and therefore polarised light of an exoplanet can be measured. The most successful planet detection method (see Figure 1.2) is the transit method, where dips in the light curve due to the movement of an planet in its foreground are measured. This method is discussed in detail below. Perryman (2018) lists further indirect or miscellaneous planet detection methods, which could be relevant in future, but their mass limitations are not sufficient investigated.

\subsubsection{Radial velocity method}

The radial velocity method to detect exoplanets is based on the reflex motion of the host star around the barycentre with a planet. This motion is measured in the radial velocity curve of the star. The first confirmed planet detection was achieved by this method (Mayor \& Queloz, 1995). Since then, the technique has proven to be a very efficient method in finding exoplanets with a steady stream of new detections (see 


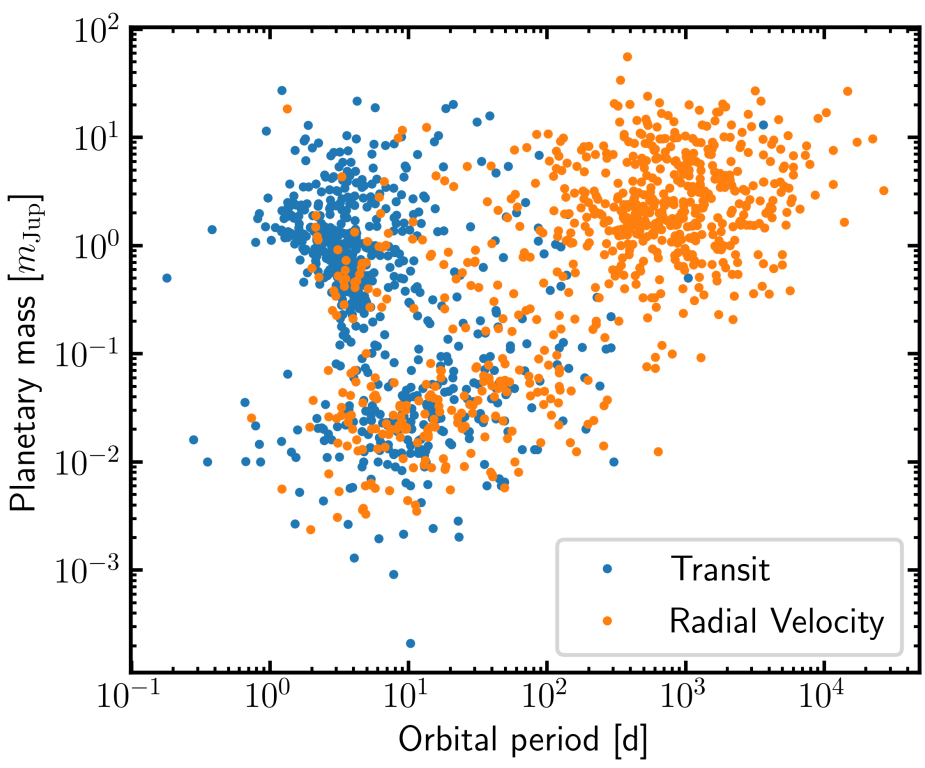

Figure 1.3: $\quad$ Planetary mass-period diagram for planets detected by radial velocity (orange) and transit (blue) measurements. The numbers are from the NASA Exoplanet Archive, 2019 September 15.

Figure 1.2.

The motion of the star around the system barycentre is measurable through a small Doppler shift of the stellar spectral lines in its spectrum. Movements towards the observer shifts the lines towards the blue and away from observer towards the red. The relation between the line shift, $\Delta \lambda$, and the (non-relativistic) radial velocity of the star, $v_{R}$, is given by

$$
\Delta \lambda \cong \frac{v_{R}}{c} \lambda
$$

with the speed-of-light $c$, and the expected line position $\lambda$.

Measuring the radial velocity of a star with an orbiting planet over a certain time yields a curve whose amplitude is described by

$$
K=\left(\frac{2 \pi G}{P}\right)^{1 / 3} \frac{m_{p} \sin i}{\left(m_{S}+m_{p}\right)^{2 / 3}} \frac{1}{\left(1-e^{2}\right)^{1 / 2}}
$$

with the gravitational constant $G$, the system period $P$, the stellar and planetary masses $m_{S}$ and $m_{p}$, the inclination $i$ and the eccentricity $e$. Given that only the radial velocity projected in the direction of the observer is measured, the inclination of the system is not derivable and so only a lower planet mass limit can be determined. The measured quantity from the radial velocity amplitude is thus $m_{p} \sin i$. From the shape of the radial velocity curve all other orbital elements are derivable, besides the position in the sky given by the longitude of the ascending node, $\Omega$, and the absolute value of the semi-major axis, $a$, the latter is not determinable due to the unknown inclination.

The most favourable planetary systems for the radial velocity detection method are heavy planets in a close orbit to its host star. Such planets feature the highest radial velocity amplitude. Nevertheless, the radial velocity accuracy of current instruments together with large telescopes allow for detections of exoplanets with a few Earth 
masses (see Figure 1.1). A mass-period diagram for the detected planets (Figure 1.3) additionally shows that planets with larger periods are also detectable when they possess enough mass.

\subsubsection{Transit method}

The transit detection method is the most effective planet detection method so far (see Figure 1.2). A transit can be measured in the light curve of a star when a planet moves in front of the star. When the planet blocks part of the star's light, the star seems to be darker for a short time. The first detection with this method was achieved in 1999, for a hot Jupiter in the HD 209458 system (Soderhjelm, 1999, Castellano et al., 2000). Hot Jupiter type planets are giant planets (radii larger than about 0.3 Jupiter radii), gaseous in nature, and in very close orbits around their host stars, thus very hot.

A transit is only observable if a planet orbits its star in the plane of the line of sight. The observation probability of a transit from a randomly-oriented planet on a circular orbit depends on the stellar radius, $R_{S}$, and the distance between planet and star, $a$ :

$$
p=\frac{R_{S}}{a} \approx 0.005\left(\frac{R_{S}}{R_{\odot}}\right)\left(\frac{a}{1 \mathrm{AU}}\right)^{-1}
$$

From transit observations many important parameters of the planet and its orbit can be derived, other than the planetary mass, which needs another measuring method like radial velocity measurements. The planetary radius, $R_{p}$, is determined by the transit depth, $\Delta F$, and its ratio to the stellar radius:

$$
\Delta F=\left(\frac{R_{p}}{R_{S}}\right)^{2}
$$

If the stellar radius is known from other measurements, the absolute value of the planetary radius is derivable as well. From the transit duration and the shape of the transit the inclination, eccentricity, and argument of periastron can be derived, as well as the distance of the planet to the star. The orbital period is known when several transits can be measured. Measuring more than two transits per planet is in any case important to verify the nature of the signal.

The transit detection method is most efficient for large planets in close orbits. The close orbits lead to a higher transit probability and the larger the planets the larger the signal. This selection effect can be seen in the planetary mass-period diagram (Figure 1.3): most of the planets detected with the transit method have periods below 100 days. With current instruments exoplanets with masses below the one of the Earth are detectable by the transit method.

An analytic model for a transit light curve was derived by Mandel \& Agol (2002). The model is based on geometric considerations of a dark sphere as a planet partly or fully covering the illuminated disk of the star. 


\section{Limb darkening}

For transit measurements it is important to consider the fact that a star is not uniformly illuminated, but darkened towards its limb. A widely used model for the limb darkening is the one published by Claret (2000). This non-linear model describes the radial brightness dependence by a fourth-order Taylor series:

$$
I(r)=1-\sum_{n=1}^{4} c_{n}(1-\mu)^{n / 2},
$$

with $\mu=\cos \theta$ and $\theta$ describing the angle between the line-of sight and the normal to the stellar surface at a certain position. A quadratic limb darkening law can be derived by fixing the coefficients $c_{1}=c_{3}=0$.

\section{The Kepler telescope}

The greatest increase in the number of detected planets from the transit method (Figure 1.2 was due to the commissioning of the Kepler space telescope. The satellite was launched on 2009 March 6 and brought to an Earth trailing, heliocentric orbit (Borucki et al. 2010). It observed a pre-selected field of stars outside of the ecliptic plane for about 3.5 years with the overall goal of evaluating the occurrence rate of Earth-like planets in the habitable zone around Sun-like stars. In the large field of view (115 sq. deg.) of the $0.95 \mathrm{~m}$ modified Schmidt telescope about 160000 stars satisfied the constrains of being monitored with brightnesses higher than 16 mag (Batalha et al., 2010). The data of the Kepler telescope are available in two cadences, long and short. Long cadence data are integrated exposures over 28.4 minutes and short cadence data for 58.9 seconds. The NASA Exoplanet Archive reports 2345 confirmed exoplanet detections by the Kepler telescope (2019 September 15). The discovered planets have radii ranging from 0.34 Earth radii to 2 Jupiter radii and periods ranging from 0.22 days to 1322 days.

The primary mission of the Kepler telescope ended in 2013 May with the failure of a second reaction wheel necessary for the quarterly turn and readjustment of the telescope to bring its solar cells in the right direction. The spacecraft could be used in the subsequent K2 mission, observing different fields for about three months each until the fuel was depleted in 2018 October 30. With the K2 mission 389 confirmed exoplanets were discovered so far (NASA Exoplanet Archive, 2019 September 15).

\subsection{Transit timing variations}

While for a single planet around a star the transits obey a linear ephemeris, in multiplanet systems the objects are gravitational interacting which can lead to deviations from this linearity. These differences in the periods of the planets are called transit timing variations (TTVs). Less susceptible, but also variable due to gravitational interactions can be the transit durations. These changes may be investigated if TTVs are measured. Their modelling is naturally considered in the photodynamical analysis 
for modelling light curves of stars with planets showing TTVs introduced in section 1.4. The measurement of TTVs is another method for detecting exoplanets and would therefore fit into the previous section (section 1.1); however, it primarily serves the confirmation and a detailed characterisation of the planets and is the main basis of this thesis, thus it deserves its own section.

Besides the possibility of confirming exoplanets only from one measurement method, the great advantage of measuring timing variations for transiting planets lies in the possibility of measuring the planets densities. From the transit a planet-to-star radius ratio is derived and from the dynamics the planet-to-star mass ratio are possibly determined. Additionally, TTVs imply a definable stellar density, $\rho_{S}$, due to its relation to the dynamical time, $t_{\mathrm{dyn}} \approx\left(G \rho_{S}\right)^{-1 / 2}$ (Agol \& Fabrycky, 2018). Together the absolute planetary densities are specified, which give a very good hint on the composition of the planets.

The idea of measurable TTVs arose from the well established modelling of eclipse timing variations in binaries. In 2002, Miralda-Escudé discussed the possibility of a second planet in the system of the first detected transiting planet HD 209458 and formulated theories of its influence on the measured planet. Two different author teams then reviewed the possibility of measuring TTVs in 2005: Holman \& Murray (2005) analysed the detectability due to TTVs of an (non-transiting) Earth-like planet orbiting a star outside of a transiting Neptune-size planet; many more planet configurations and their measurable TTV amplitudes were approximated by Agol et al. (2005). These configurations will be discussed in detail later in subsection 1.2.3, where different types of TTVs are outlined. In 2008, Nesvorný \& Morbidelli looked into the possibilities of determining masses and orbits from TTVs of exoplanets. At that time, all TTV work was only theoretical and there had not been a measurement. Transit detections were still only performed from ground and only hot Jupiter type planets had been detected. Later it was found out that hot Jupiters lack close companions that could induce TTVs (Steffen et al., 2012). The picture changed when the Kepler space telescope was launched in 2009 (see section 1.1.2). With Kepler-9 (Holman et al. 2010) the first multiple transiting system was found and directly confirmed via its TTVs. This system is the object of an analysis in this thesis, the results from its dynamical analysis can be found in chapter 2 .

Today, 305 planets showing TTVs in 175 planetary systems are discovered. 165 of the planets have determined masses and only a fraction of the systems are characterised in detail (NASA Exoplanet Archive, 2019 September 15). The reason for the low numbers of fully characterised TTV systems is, on the one hand, the timeconsuming dynamical analysis, which involves numerical simulations of the system in most cases, and, on the other hand, the often long periods of TTV signals preventing the measurement of full TTV cycle. Another factor is that many systems contain only one TTV planet. In such systems, the mass of this planet is not derivable, yet the detection of another planet inducing these TTVs might be possible. For filling the TTV cycle with data points, even after 4 years of primary Kepler observations, follow-up observations are necessary. One project dedicated to performing groundbased follow-up observations to fill the dynamical cycle of TTV systems is the KOINet 
(see section 1.3), where this thesis originated.

Before diving into the details of TTVs, their periodicity and amplitudes, a few fundamentals must be covered. Therefore, the next two subsections deal with multiplicity in planetary systems and mean-motion resonances.

\subsubsection{Multi-planet systems}

Transit timing variations due to gravitational interactions only appear in multi-object systems. Most often such systems consist in a host star orbited by multiple planets. The NASA Exoplanet archive lists 4055 (3117 transiting) confirmed exoplanets in total, 2338 (1782) of them are in single planet systems and 1717 (1335) in 681 different multi-planet systems. These multiple systems split into 452 two-planets systems, 143 three-planets systems, 57 four-planets systems, 21 five-planets systems, 6 six-planets systems, 1 seven-planets system, and 1 eight-planets system (Numbers from 2019, September 15). Both of the systems with seven and eight planets, were found by the transit detection method. In the following a few differences in the nature of planets in single and multiple planet systems are examined. Only transiting planets will be considered as these are the systems of interest for TTV analysis. Apart from that the different biases of different detection methods makes the comparison difficult.

In Figure 1.4 the number of planets over the semi-major axis (top), the planetary radius (middle), and the planetary mass (bottom) for transiting planets in single and multiple planetary systems are plotted. The multiple planet systems are not differentiated in the systems with different numbers of planets as it makes no big difference and the abundance of detected systems decreases with the number of planets in the system, resulting in a lack of statistical evidence for the many-planets systems. Planets in single systems tend to be closer to its host star, peaking at about $0.05 \mathrm{AU}$, whereas planets in multiple systems have a broader distribution in semi-major axis and peak at about 0.1 AU. This distribution is not influenced by the stellar host parameters: the distribution of planets in single and multiple systems are similar for stellar radii and effective temperature. The planet radii distribution shows a more significant difference between single and multiple systems. Planets in single systems are either in the regime of Super-Earths peaking at about $0.2 R_{\mathrm{Jup}}$ or in the regime of giant planets with radii of the order of Jupiter's radius. In multiple systems, only a very small fraction of giant planets are found. The distribution peaks like that of the single system regimes at about $0.2 R_{\text {Jup }}$. The masses of transiting planets are not measurable from a transit observation. So, only a small fraction of the planets detected by the transit method have determined masses. For planets in single systems, the mass determination of the planets is most often conducted through radial velocity measurements, which are more feasible for massive planets. Hence, the large transiting single planets have determined masses which peaks at about one Jupiter mass. For transiting planets in multiple systems the masses can also be derived from TTVs if detected. Those systems have planets with significant lower masses, peaking at about $0.03 m_{\text {Jup }}$.

All these observations together suggest that a large proportion of the planets 

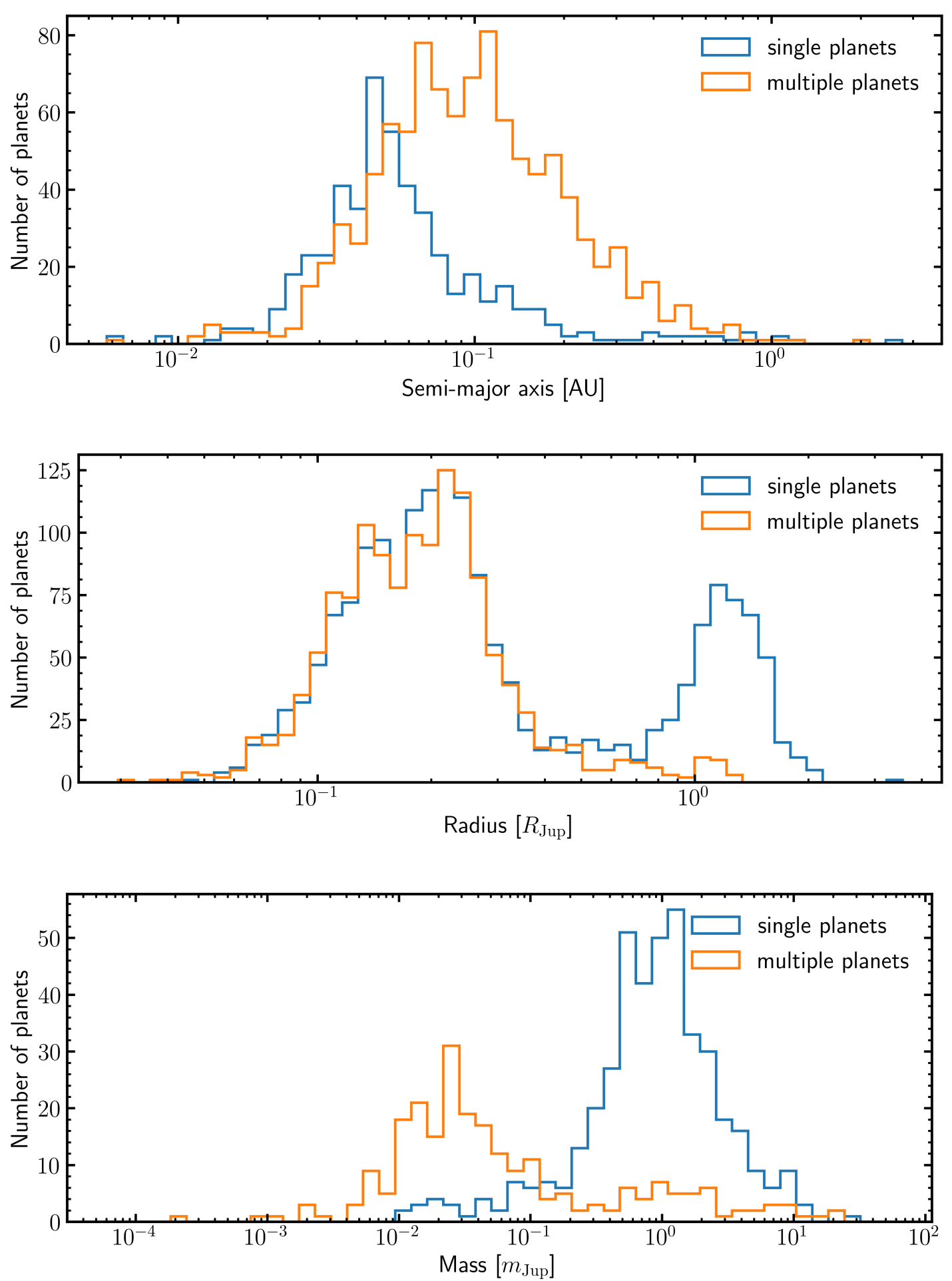

Figure 1.4: The number of planets over the semi-major axis (top), the planetary radius (middle) and the planetary mass (bottom) for transiting planets. The data is subdivided into planets in a single planet system, blue, and planets in a multiple planet system, orange. The numbers are from the NASA Exoplanet Archive, 2019 September 15. 
detected by transit measurements in single systems are hot Jupiters. These are rarely found in multiple systems suggesting that close-in giants either disrupt the inclination of small planets in the system and lower their transit probability, directly prevent their formation from the beginning or have resulted in the destruction of the other planets due to their orbital evolution from a "cold" to a "hot" Jupiter. This observation matches the result by Steffen et al. (2012), who found out that hot Jupiters have no near companions that could induce TTVs. This fact also eliminates the possibility of near planet companions in slightly inclined orbits.

Studies on the architecture of transiting multi-planet systems are performed by several authors. In 2013, Ciardi et al. studied Kepler multi-planet systems and found a tendency in planet pairs that are of the order or larger than Neptune for the larger planet to be outside of the smaller planet. They found no such tendency in planet pairs of smaller size. Weiss et al. (2018) detected a 'peas in a pod' behaviour, meaning the planets in multiple systems are more similar in its size than expected if the sizes were randomly selected. A similar behaviour was found by Millholland et al. (2017). They found the same tendency not only for the radii, but also for the masses of the planets by considering only TTV planets where masses are determined.

The co-planarity of multi-planets systems was investigated relatively early on when most of the planets were detected by radial velocity measurements. In these systems the inclination of the planets is not determinable, hence there is no knowledge about the co-planarity of the systems. The research was done with the help of numerical simulations suggesting a large proportion of planetary systems containing giant planets are highly unlikely to be co-planar (e.g., Thommes \& Lissauer (2003), Adams \& Laughlin (2003), and others). Mechanisms leading to these systems can be the collision scattering of protoplanets or a fast amplification of relative inclination occurring at resonances of first order (this terminology is introduced in the next section). This investigation could explain the lack of giant planets in multi-transiting planet systems. Either the giant planets are highly inclined and outside of the transiting region or the systems do not contain giant planets as they would disrupt the inclination of all the planets.

Recently, Sandford et al. (2019) published a study of the multiplicity distribution of exoplanet systems detected by the Kepler telescope around FGK type stars. They found a Zipfian (Zipf, 1935) distribution to match the measured multiplicity when applying biases given by the detection method and the instrument. This model implies that the true number of planets around the stars is about $2.22 \%$ higher than the measured one and that every second single planet system actually contains another planet. Reasons for these planets to be not detected are for example the absence of co-planarity or small radii.

The planet multiplicity of systems in the context of TTV measurements was investigated by Kane et al. (2019). They visually examined the TTV curves of all planets and planet candidates detected by Kepler that are listed in the TTV catalogues by Rowe et al. (2014) and Holczer et al. (2016). From the visual inspection they classified the TTVs by their cleanliness and several other factors leading to a sample of objects that are showing strong TTVs (overall rating 8-9). For these objects they found out 
that they are $1.6 \pm 0.2$ times more frequent in planetary systems containing three or more planets than in systems with only one or two systems.

With the results by Kane et al. (2019) a correlation analysis for TTV measurements similar to Mazeh et al. (2013) is possible. Mazeh et al. (2013) found a correlation of 0.48 between the orbital period of a planet and its TTV period and a correlation of 0.51 between the TTV period and the TTV amplitude of the planets. In Figure 1.5 the correlation between the TTV period and the orbital period of the planets where Kane et al. (2019) found strong TTVs for single and multiple systems is plotted on the top and the correlation between the TTV amplitude and the TTV period respectively on the bottom. A calculation of the Pearson correlation coefficient yields a value of 0.18 for the correlation between the TTV period and the orbital period $(0.20$ for planets in single systems and 0.35 for planets in multiple systems). The correlation between the TTV amplitude and the TTV period is 0.24 (0.08 for planets in single systems and 0.44 for planets in multiple systems). Both correlations are less significant than the ones found by Mazeh et al. (2013). The reason for this discrepancy could be the smaller observation baseline of the Mazeh et al. (2013) analysis leading to possibly wrong TTV period values, given the fact that even the whole Kepler observation time is in some cases too short to cover a full TTV cycle. Another point is the smaller sample of Mazeh et al. (2013) in comparison to the analysis by Kane et al. (2019).

\subsubsection{Mean-motion resonances}

Resonances can occur in multi-planet systems and if so they highly influence the dynamics of the system. Orbital resonances appear when there is a simple relationship between periods or frequencies, resulting in regular, periodic gravitational influences that can lead to unstable orbits when the planets exchange momentum and their mutual orbits change. In other cases the resonances lead to mutual self-correcting orbits and stabilise each other. These planets remain in the resonance for an indefinite period of time.

A mean-motion resonance does not only require a certain orbital period ratio, but a regularity defined by a linear combination of the orbital angles. The resonance is true if the angle combination is librating instead of circulating. Hereby, libration means the presence of oscillating angles, and circulation the absence of such oscillations. For a pair of planets with a period ratio near commensurability,

$$
\frac{P_{1}}{P_{2}} \simeq \frac{p}{p+q},
$$

where $p, q$ are integers and $q$ defines the resonance order of the mean-motion resonance. For this system the resonant angles are given by

$$
\phi=(p+q) \lambda_{2}-p \lambda_{1}-q \tilde{\omega}_{1,2}
$$

with the mean longitude, $\lambda$, and the longitude of the periastron, $\tilde{\omega}$. In order for the planets to resonate, these angles must librate. 

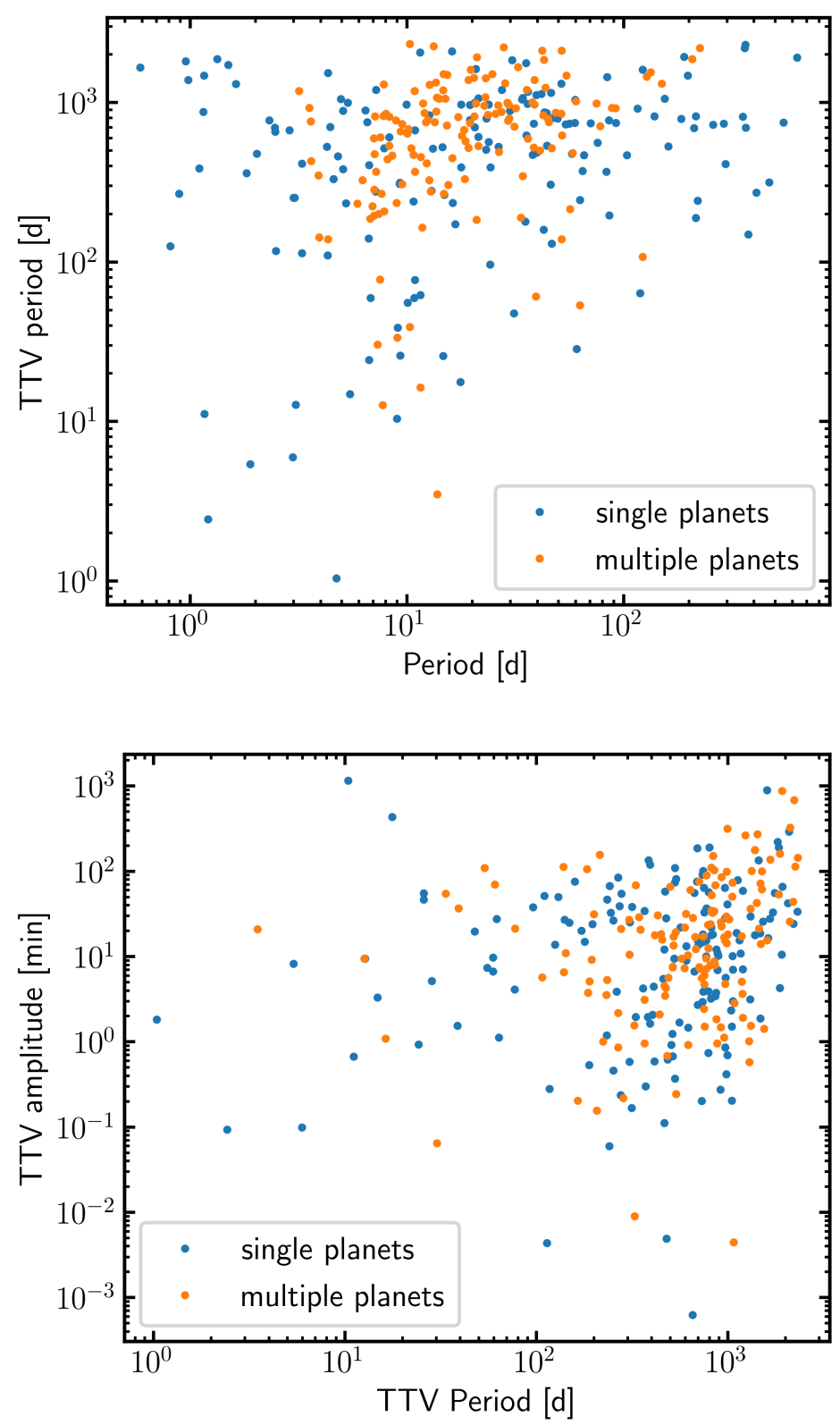

Figure 1.5: Correlation of TTV period with orbital period (top) and TTV amplitude with TTV period (bottom). Blue points indicated the parameters for planets in single systems and blue points the ones for planets in multiple systems. The data are the strong TTV planets from Kane et al. (2019). 
In the sample of planet systems detected with the Kepler telescope, it is observed that only a small fraction of planet pairs have a period ratio close to a low-order commensurability. This number, however, is higher as if the period ratios have been randomly chosen from the range of appearing period ratios (Lissauer et al., 2011). The planet pairs showing near period commensurabilites can be categorised in three different of resonance-related relationships: (1) the most common planetary pairs are those that have a period ratio that is from one to several percent larger than a nearby commensurability; (2) some planet pairs lie within one per thousand near an exact period ratio; (3) the period ratio of a few planet pairs differ by about one percent from a commensurability, but are in mean-motion resonance because their resonance angles are librating.

\subsubsection{Different types of TTVs}

The timescale of the gravitational interaction of planets is of the order their orbits, suggesting a scaling of the TTV amplitude with the orbital period. All other contributions to the amplitude are dimensionless. Known from Newton's law is the independence of gravitational effects on an object from its own mass. For this reason the TTV amplitude only depends on the masses of the other system objects. For a two-planets system the TTV amplitudes are given by

$$
\begin{aligned}
& \delta t_{1}=P_{1} \frac{m_{2}}{m_{0}} f_{12}\left(\alpha_{12}, \theta_{12}\right) \\
& \delta t_{2}=P_{2} \frac{m_{1}}{m_{0}} f_{21}\left(\alpha_{12}, \theta_{21}\right)
\end{aligned}
$$

with the periods $P$, the masses of the star and the planets $m_{0}, m_{1}$ and $m_{2}$, and a function, $f_{i j}$, describing the perturbation of planet $j$ on planet $i$. The perturbation is a function of the semi-major axis ratio $\alpha_{i j}=\min \left(a_{i} / a_{j}, a_{j} / a_{i}\right)$, and the angular orbital elements of the planets, $\theta_{i j}=\left(\lambda_{i}, e_{i}, \omega_{i}, I_{i}, \Omega_{i}, \lambda_{j}, e_{j}, \omega_{j}, I_{j}, \Omega_{j}\right)$. These formulae have been studied by numerous author teams with the help of perturbation theories aiming for analytic solutions to analyse TTV planet systems (see e.g. Nesvorný \& Morbidelli, 2008, Nesvorný \& Beaugé, 2010, Agol \& Deck, 2016, Deck \& Agol, 2016). The detailed examination of the studies is beyond the scope if this thesis, because here the dynamical analysis is based on numerical integrations of the planetary systems, instead of an analytic approximation.

In a system with multiple perturbing planets the TTV amplitude can be expressed as a linear combination of the perturbations per planet if the planet-to-star mass ratios are sufficient small and in the absence of resonances. Systems with more than two planets, where this is not given, need to be analysed by the help of a numerical integration.

\section{Planets in mean-motion resonance}

Planets that are in mean-motion resonance show the strongest TTV amplitudes. The timing variations are the results of a librating semi-major axis. For a planet pair 
initially on circular orbits and in first order resonance with the period ratio given by $j: j+1$, Agol et al. (2005) found a maximum TTV amplitude of

$$
\delta t \sim \frac{P}{4.5 j} \frac{m_{\text {pert }}}{m_{\text {pert }}+m_{\text {trans }}},
$$

with the libration period, $P$, and the masses of the perturbing planet, $m_{\text {pert }}$, and the transiting planet, $m_{\text {trans }}$.

\section{Non-resonant planets on circular orbits}

For a planet pair on circular obits in a non-resonant period ratio the TTV amplitude is still driven by the resonance term. The interaction of the planets is largest at the points of conjunction. At this point the planets perform a radial kick on each other ending in an eccentricity change which causes a change in the semi-major axis and the mean motion. The period of the TTV interaction for a planet pair with a period ratio $P_{2} / P_{1}$ from the outer planet to the inner close to $m / n$, with $m$ and $n$ being integers is given by

$$
P_{\mathrm{TTV}}=\frac{1}{\left|m / P_{2}-n / P_{1}\right|} .
$$

This period is also called the 'super period'. The amplitude of the TTV is smallest half-way between two resonances. There, it is given by the mass ratio of the perturbing planet to the star, the period of the transiting planet and the squares of the ratio of the transiting planet semi-major axis to the difference between the transiting and the perturbing planets semi-major axis. For a pair of planets the TTV curves are sinusoidal and anti-correlated due to the conservation of energy and angular momentum.

\section{Perturber: inner planet on a small orbit}

In this case there is no planet-planet interaction. Instead, the inner planet acts on the host star, both are orbiting the common barycentre. The transit of the outer planet can happen at different positions of the host star, which is changing the timing of the transit. The resulting variation amplitude for circular, co-planar, and edgeon observed orbits depends on the mass ratio of the inner planet to the star, the semi-major axis ratio of inner planet to outer planet, and the period of the outer planet (Agol et al., 2005). The variation vanishes if the period of the outer planet corresponds to a whole multiple of the period of the inner planet. In this case the transit always happens at the same position of the star.

\section{Perturber: massive outer planet with a large eccentricity}

For a two planet system with a transiting inner planet on a circular orbit and a massive outer planet with a much larger semi-major axis and in an eccentric orbit the planet-planet interaction induces the transit timing variations. The variable distance between the planets leads to a change in the period of the inner planet. The TTV 


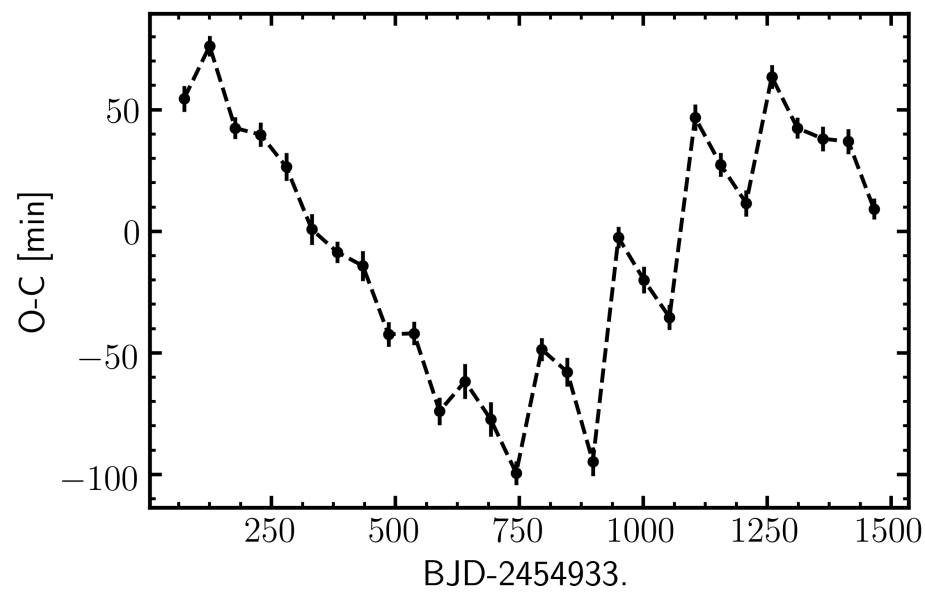

Figure 1.6: $\quad$ Transit times $(\mathrm{O})$ minus a linear ephemeris (C) over observation time of Kepler-82c. The dashed line connects the measurement points for better visualisation of the saw tooth profile of the chopping component in the TTVs.

amplitude is dependent on the period, eccentricity, and the mass ratio to the host star of the outer planets, as well as the cubes of the semi-major axis ratio of outer planet to inner planet (Agol et al., 2005).

\section{Chopping}

As previously mentioned, the interaction between two planets is highest at their conjunction. This interaction also induces a small period and small amplitude TTV signal. At conjunctions the inner planet is slowed down and the outer planet is sped up. This change in the speeds results in a saw tooth like TTV profile, therefore the effect is called 'chopping' (e.g., Holman et al., 2010, Deck \& Agol, 2015). In Figure 1.6 the TTVs of Kepler-82c are plotted as an example for chopping TTV profiles. The graph shows an O-C diagram typically used for presenting TTVs. The transit times (observed, O) are subtracted by a linear ephemeris (calculated, C), remaining are the variations. Underlying a larger amplitude, larger TTV period trend is the saw-tooth profile of the chopping component with a jump every three consecutive transits.

The period of a chopping component in TTVs is given by the period of conjunction, also called synodic period

$$
P_{\text {syn }}=\frac{1}{\left|1 / P_{1}-1 / P_{2}\right|} .
$$

Measuring a chopping component in the TTVs of a planet is very valuable as it leads to the measurement of the absolute mass of the perturbing planet (Nesvorný \& Vokrouhlický, 2014, Deck \& Agol, 2015).

\subsection{The KOINet}

The Kepler Object of Interest Network (KOINet) ${ }^{2}$ is a network of ground-based telescopes dedicated on follow-up observations of TTV planets and planet candidates de-

\footnotetext{
${ }^{2}$ http://koinet.astro.physik.uni-goettingen.de/
} 
tected in the Kepler primary mission. The life time of the latter was not long enough for all detected TTV planets to fill the full dynamical cycle of the TTV curves. One aim of the KOINet is to fill the dynamical cycle with the help of the follow-up observations. Subsequent goals are the dynamical characterisation of the planetary systems with help of a photodynamical model (see section 1.4), the confirmation of some of the planet candidates and the detection of new, possibly non-transiting planets. The structure, observation strategies and handling of the ground-based observations are described in von Essen et al. (2018). The following section summarises these insights. Furthermore, the publication shows first results of the KOINet in terms of measured transit times of four different targets from the KOINet. Other targets, where the Kepler data are complemented by KOINet data and analysed, are the Kepler-9 system (Freudenthal et al., 2018) and the Kepler-82 system (Freudenthal et al., 2019). The photodynamical analysis of these system are the essence of this thesis and the publications can be found in chapter 2 (Kepler-9) and chapter 3 (Kepler-82).

The KOINet has a list of targets that are interesting for the ground-based followup observations. These targets are picked from the Kepler TTV catalogues by Ford et al. (2012), Mazeh et al. (2013), Xie (2013, 2014), Nesvorný et al. (2013), Ofir et al. (2014) and Holczer et al. (2016). Thereby, two criteria were taken into account to ensure a sufficient photometric precision, resulting in a sufficient timing precision, and to avoid too high correlated noise in the data to be able to measure transits: the transit depth has to be larger than one part per thousand and the timing variability of the planet has to be greater than two minutes. With these criteria a list of sixty TTV planets and planet candidates were assembled and categorised in four different priority types (ordered): (1) polynomial shaped TTVs with anti-correlated behaviour between two planets, where any additional point could show the turnover time from a parabolic to a sinusoidal-like shaped TTV curve, which leads to a more accurate mass determination; (2) sinusoidal-like shaped TTV objects that are anti-correlated between a planet pair but poorly sampled, where additional transit measurements could improve the dynamical analysis; (3) objects with very long TTV periods; where new transit measurements could give a hint on the actual period of the TTVs; (4) planets that are already well-characterised or systems where only one object is showing TTVs.

For the ground-based observations the network has access to 18 telescopes located at 16 observatories on the Northern hemisphere around the globe. These telescopes have apertures between 0.6 - 6.5 meter diameter. All observations are carried out with an R-band filter, which reduces the impact of the Earth's atmosphere on the photometry by avoiding telluric contamination in the I-band and the absorption of light at shorter wavelenghts. Additionally, the R-band filter ensures rather small light curve variations induced by stellar spots and the limb darkening. Furthermore, the observations are slightly defocused to minimise the noise in the photometry (Kjeldsen \& Frandsen, 1992, Southworth et al. 2009). For the same purpose an engineered diffuser (ED) (Stefansson et al., 2017, 2018a,b) is another option that reduces the photometric noise even more. Here, the incoming photons are spread in a more homogeneous way than when defocusing, resulting in broad, homogeneously illuminated 
point spread function for the observed stars independent of seeing changes. Recently, two different ED's were installed at the 2.5 meter Nordic Optical Telescope $\mathrm{f}^{3}$ (von Essen et al., 2019). This will improve the time measurements of KOINet targets in the future when observed by the telescope. For the data reduction, calibration frames (bias and flat fields, in rare instances also dark fields) are carried out each observing night.

To provide estimated transit times for scheduling the observations, different models are fitted to the transit times from Kepler observations according to the shape of the TTVs. For complex TTVs the predictions are taken out from a dynamical model if available. In other cases the TTVs are simply fitted by a linear ephemeris and a sinusoidal term. If the turnover in the TTVs is not yet measured, two different predictions are given, one by fitting a linear epheremis plus a sinusoidal term and the other from fitting a linear ephemeris and a parabola term. For objects which show TTVs with no specific shape, only the linear ephemeris is fitted.

\subsubsection{Light curve extraction}

The data reduction of KOINet observations are performed by the DIP ${ }^{2}$ OL (Differential Photometry Pipelines for Optimum Lightcurves) reduction pipeline. The tool consist of two parts, one is based on IRAF-tools and the second is python-based.

The first step with IRAF is the calibration of the science frames. For this, master bias, dark, and flat frames are generated by averaging over all respective calibration observations if available. The science frames are subtracted by the bias and dark master frames and divided by the flat master frame if applicable due to availability. Afterwards, a cosmic ray rejection procedure is applied to the target observation frames. The third part in the IRAF procedure is the measurement of the fluxes for the target star and reference stars in several different apertures and sky rings. The aperture and sky ring diameters are set into proportion to the intra-night averaged full width at half maximum. Further values are extracted necessary for the later detrending of the photmetric light curve. These are the seeing, the airmass at the center of the field of view, the $\mathrm{x}, \mathrm{y}$-centroid positions of the target star and all reference stars, the sky values for each sky ring and measured star and the integrated fluxes of the master flat and the master dark if available per aperture and measured star.

The first step afterwards in python is the selection of the optimal combination of the reference stars, the aperture diameter, and the width of the sky ring to minimise the scatter in the photometric differential light curve. The scatter, thereby, is measured by a spline function for the light curve since the existence of a transit in the observation time is not known at this point. Another procedure in python is the transformation of the time stamps from the science frames from Universal Time to Barycentric Julian Dates in Barycentric Dynamical Time (BJD ${ }_{\mathrm{TDB}}$ ) using a pythonwrapper for the web tool by Eastman et al. (2010) The detrending and transit modelling of the differential light curve is also part of the python-based DIP² OL part,

$\sqrt[3]{\text { http://www.not.iac.es/instruments/alfosc/diffuser.html }}$

${ }^{4}$ http://astroutils.astronomy.ohio-state.edu/time/utc2bjd.html 
but it no longer relates to the light curve extraction and is therefore described in the following section.

\subsubsection{Detrending and transit modelling}

For the detrending and the transit modelling of the extracted KOINet observations, initial transit model parameters of good precision are necessary. The determination of them is described in the first part of this section.

Part of this thesis project was a contribution to the KOINet by providing initial transit parameters for the sixty planets and planet candidates. The parameters are derived from modelling all transits of the objects in the Kepler long cadence data. To reduce the impact of the TTVs in the data every two consecutive transits are modelled simultaneously. From the Kepler data the transits are extracted with one transit duration out-of-transit data before and after the transit. For detrending the observations, the outside transit data belonging to one transit are modelled by a second-order polynomial and the extracted light curve is divided by this fitted function. The modelling of every two consecutive transits simultaneous is done with the transit Mandel \& Agol (2002) model assuming a quadratic limb darkening law. The limb darkening coefficients are extracted from the values calculated by Claret et al. (2013) for the fundamental stellar parameters of the objects host star (effective temperature, metallicity, surface gravity) from the NASA Exoplanet Archive. To reduce the impact of the sampling rate on the derived transit model parameters (Kipping, 2010), the model is computed on a fine grid of thirty points per observation time step and rebinned to the data points afterwards. Assuming circular orbits, the transit modelling delivers values for the semi-major axis, orbital inclination, planet-to-star radius ratio, orbital period, and mid-transit time. The parameter space is explored by the Markov chain Monte Carlo (MCMC) algorithm PyMC (Patil et al. 2010$)$ accessed from the PyAstronomy ${ }^{5}$ package and the mean and standard deviation of the MCMC posterior distribution gives the fitting parameters. The initial input transit parameters used to model the ground-based observations are derived from the mean and standard deviation of the model parameter distribution of analysing all two consecutive transits of an object. For KOINet objects for which a dynamical modelling like a photodynamical analysis (see section 1.4) has already been performed, the transit parameter predictions of this analysis are used as initial parameters for the KOINet observation modelling.

For extracting the transit times of ground-based data by modelling the transit, a more refined detrending model than just a polynomial is necessary to carefully respect the influence of the Earth's atmosphere and the individual instruments of the observations. The detrending model is also important for the photodynamical analysis section 1.4) when including KOINet observations. For this reason, a more complicated model was developed in cooperation with C. von Essen as part of this thesis project. Due to the high time consumption of the photodynamical model by numerical integrations, the detrending model needed to be fast. Therefore, a linear combination of an extraction of components that induce trends in the photometric

${ }^{5}$ http://www.hs.uni-hamburg.de/DE/Ins/Per/Czesla/PyA/PyA/index.html 
light curve is calculated for a given transit model. The possible detrending components are the seeing $\hat{S}$, airmass $\hat{\chi}$, and per measured star, $i$, the $X_{i}, Y_{i}$-centroid positions, sky background counts $B G_{i}$, and flat and dark fluxes, $F C_{i}$ and $D K_{i}$. The full detrending model $(D M)$ when considering all components has the form

$D M(t)=c_{0}+c_{1} \cdot \hat{\chi}+c_{2} \cdot \hat{S}+\sum_{i=1}^{N+1} x_{i} \cdot X_{i}+y_{i} \cdot Y_{i}+b g_{i} \cdot B G_{i}+f c_{i} \cdot F C_{i}+d k_{i} \cdot D K_{i}$

with $N+1$ denoting the total number of stars, target and reference, and the coefficients of the model are $c_{0}, c_{1}, c_{2}, x_{i}, y_{i}, b g_{i}, f c_{i}$, and $d k_{i}$. The coefficients are calculated from this linear combination while considering a transit model for the photometric light curve. In order not to overfit the observations, not all possible detrending components are considered, but a sub-model is searched that sufficiently detrends the data with a minimum amount of detrending components. The individual star components can be considered for only the target star, for all stars or as a combination of all stars. To determine the best matching detrending model of an observation an array of trial transit mid-times from the predicted time plus/minus the transit duration is arranged. A transit Mandel \& Agol (2002) model with the initial transit model parameters of the object and quadratic limb darkening law together with a detrending model is calculated. The quadratic limb darkening coefficients are computed for the Johnson-Cousins R-filter transmission response and angle-resolved synthetic spectra from spherical atmosphere models using PHOENIX (Husser et al., 2013) with stellar parameters closely matching the ones of the star (see, e.g. von Essen et al., 2013). With the combined model a minimisation statistic to the data is computed for the transit time array and the sub models with the different combinations of detrending components. The mean of four different minimisation statistics is used to ensure a good number of fitting parameters. These statistics are the reduced- $\chi^{2}$, the Bayesian Information Criterion, BIC, the standard deviation of the residuals enlarged by the number of fitting parameters, and the Cash (1979) statistic. For a trial transit midtime near the actual transit time, all statistics should be minimised independent of the chosen detrending model. In this way, the detrending model which most minimises the mean of the statistics at the transit time is chosen, while taking the number of detrending components into account. In Figure 1.7 two different KOINet light curves and there detrending models are visualised as an example. The top plot shows an example where large trends and jumps due to observation breaks are corrected by the detrending procedure. A full transit observation and the correction of small effects with the detrending, resulting in a reduction in the scatter in the light curves is visible in the bottom plot.

The last part of DIP ${ }^{2} \mathrm{OL}$ contains a transit fitting routine for transit time determination only when this value is of interest and for accurate errorbars that ensure a correct subsequent use of the ground-based light curve in, for instance, the photodynamical modelling. To calculate accurate errorbars, correlated noise in the observations needs to be considered (see e.g. Carter \& Winn, 2009). The observed light curve is fitted with an MCMC algorithm by the transit Mandel \& Agol (2002) model with quadratic limb darkening coefficients combined with the fitted detrending 

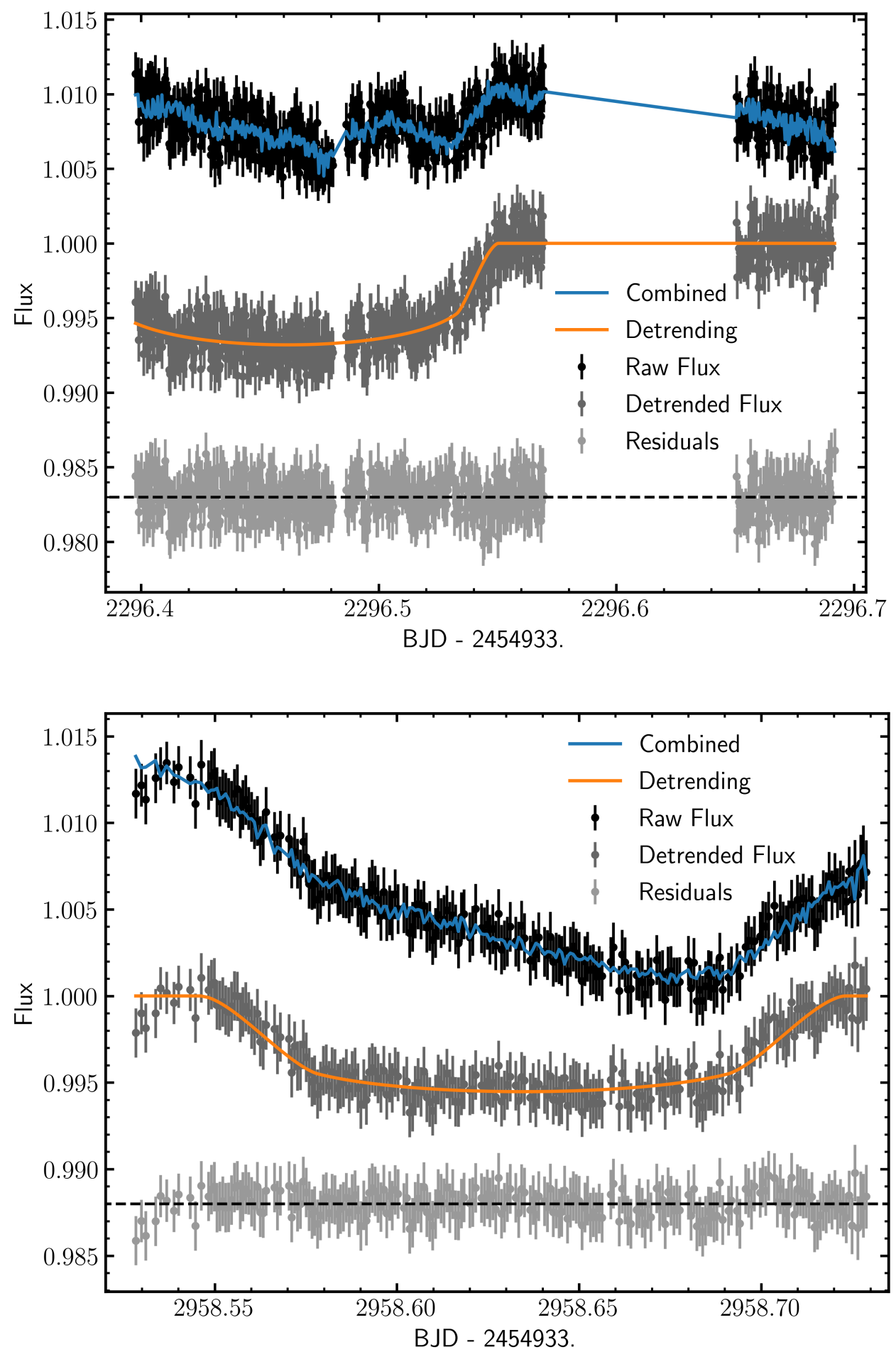

Figure 1.7: Two different examples of KOINet light curves to show the detrending. The plots show from top to bottom the raw light curve with the combined detrending and transit light curve model, the detrended light curve with the transit model and the residuals of the data from the combined models. The curves are artificially shifted for better visualisation. Both plots show KOINet light curves of Kepler-9 with a transit of planet c (top) observed by the $2 \mathrm{~m}$ Liverpool telescope (Steele et al., 2004) and a transit of planet b (bottom) observed by the $2.5 \mathrm{~m}$ Nordic Optical Telescope. 
model. The transit model is initialised with the determined transit parameter of the object, the transit time that minimised the statistics during the detrending model determination, and the calculated limb darkening coefficients for the Johnson-Cousins R-filter. A full fit with semi-major axis, inclination, planet-to star radius ratio and with the mid-transit time as free parameters is only performed if the transit curve is fully covered by the observation. Thereby, all parameters other than the transit time have Gaussian priors during the MCMC optimisations with the mean and standard deviation values from the parameters fitted on Kepler data. If the transit is not fully covered, only the transit mid-time is fitted to avoid corruption due to false fitted transit shape parameters. To check the light curve for correlated noise the $\beta$ factor (von Essen et al., 2013, 2018) is calculated in time bins relating to the transit ingress duration on the residuals from subtracting the best fit from the light curve. If the $\beta$ value is larger than one, the errorbars are enlarged by the factor and the transit light curve fitting is repeated in the same fashion. The raw light curve, corrected errorbars and all detrending components are saved for later analysis with, for example, the photodynamical model.

\subsection{Photodynamical modelling}

The analysis of KOINet objects in order to characterise the planetary system was the main part of this thesis project. Therefore, a photodynamical model was developed and optimised for all transit light curve observations of a system. This analysis method combines an n-body simulation of the system over the time span of observations with an analytic model for the transit light curves. Ragozzine \& Holman (2010) first mentioned the advantages of a photodynamical model when analysing transiting multi-planet systems. The authors performed a theoretical analysis and reached the following conclusions:

- there are many local minimas in the parameter space, making it difficult to find the global minimum if the optimisation algorithm is not started close to it;

- the mass ratios and the semi-major axis of the planets can be determined very precisely if TTVs play a dominant role in a multi-planet system;

- measuring the dynamical masses of the planets by the light-travel time effect would need a much longer observation baseline than given by the Kepler telescope;

- for measuring limb-darkening coefficients of the star the combination of several transiting planets in a simultaneous optimisation gives no significant advantage the largest planet in the system mostly defines the limb darkening measurement.

Especially the possibility of a precise mass ratio determination gives a high motivation to model TTV planet systems photodynamically. The difficulties of finding the global optimisation minimum, can be solved by conducting a TTV analysis as a first step to approximate the system dynamics and then using the results as the input for the light curve analysis. 


\subsubsection{The algorithm}

The n-body simulation is performed with the second-order mixed-variable symplectic (MVS) algorithm implement in the Mercury6 package by Chambers (1999). Symplectic integrators like this are often used for astrophysical problems because of their speed in comparison to conventional integrators and the absence of long-term accumulation of the energy error. The MVS integrator is faster than the more accurate Burlisch-Stoer (BS) algorithm implemented in the same package, but still applicable. The BS algorithm is a hybrid of a symplectic and a conventional integrator. This has the advantage that close encounters can be integrated more accurately with the conventional integrator and another advantage of this integrator is the precision in high-frequency terms of the Hamiltonian. The former is insignificant, there are no close encounters in the KOINet systems. The latter was discussed by Deck et al. (2014) as an advantage of BS integrators. For the application in the photodynamical model a test was performed by comparing the results of both integrators. In the time span of available observations the differences are negligible, errors of the order of the time step of observations occur after about 50 years of integration time. The MVS integrator has a sufficient precision for the utilisation in the photodynamical model applied to KOINet objects. A first-order post-Newtonian correction (Kidder, 1995) was added to the integrator to account for non-Newtonian gravitational forces. To accelerate the integration by avoiding input/output overhead, a python-wrapper for Mercury6 was written (Husser, priv. comm.).

From the numerical integration the planet positions are extracted to calculate projected distances between the planets and the star centres. The plane of sky is defined as the $\mathrm{x}-\mathrm{y}$ plane in the Mercury6 output. Therefore, the distances are calculated from the $\mathrm{x}$ - and $\mathrm{y}$-coordinates. The line-of-sight, in which a transit happens, is defined as the positive z-axis. With the distances the transit Mandel \& Agol (2002) model is calculated respecting the quadratic limb-darkening law implemented in the occultquad routine. The light-travel-time effect is considered by individually correcting the Mercury6 output time with the distance of the planets from the star in the line-of-sight.

For KOINet data the earlier described detrending calculation (see subsection 1.3.2) is applied after the photodynamical model computation.

\subsubsection{Data-algorithm correspondence}

For the photodynamical model only the parts of the light curves where transits occur are important. Therefore, from Kepler data the transit light curves with a certain portion before and afterwards are extracted and joined to one input light curve. The KOINet data already comprise only a low number of outside transit data and certain parts of the transit and are used as a whole.

The Kepler long cadence data have a time resolution of about 30 minutes which is of the order of the ingress/egress time of most of the KOINet transits. This has a significant impact on the derivation of the transit parameters if the model is calculated with the same time step directly (Kipping, 2010). For this reason the photodynamical 
model is oversampled with about 1 minute cadence for long cadence data and rebinned to the actual data points.

For minimising the time consumption of the numerical integration by Mercury 6 and its processing, a first system simulation is done on a coarse grid. The step size of this grid is optimised to grant the shortest possible model computation time with sufficient accuracy. To ensure good accuracy, the time step must not exceed a twentieth of the period of the innermost planet. For phases near a transit a detailed integration is done with a step size of 0.01 days. This step size assures a light curve accuracy of 0.01 parts per million for Kepler long cadence data. The accuracy is determined by the comparison of a model calculated with the given step size and the same model calculated with half the step size.

Neither the sampling on a coarse grid, nor on the fine grid matches the time steps of the observations. For this reason, the projected distances over Mercury6 time in the vicinity of a transit is modelled with a hyperbola to interpolate the result. The Mandel \& Agol (2002) model finally is calculated at the observation times with the interpolated distances, quadratic limb-darkening coefficients and the planet-star radii ratio.

\subsubsection{Model parameters}

Before the photodynamical model is optimised on the light curve, a linear ephemeris is fitted to the transit times $T$, over its ephemerids $n$, of each planet $p$ :

$$
T=\Delta T_{p, 0}+P_{p} \cdot n
$$

with the offset $\Delta T_{p, 0}$ between the zeroth transit and the chosen reference time for starting the numerical integration, and the mean period $P_{p}$. These parameters are used in the photodynamical model for calculating a starting value of the semi-major axis and the mean anomaly described below.

The choice of model parameters was guided by the modelling rather than by the observations, meaning for instance Mercury6 takes the semi-major axis of the planets as input parameter, whereas the data deliver the periods. In the following the model input parameters are described. For the central star the mass $m_{S}$, and the radius $R_{S}$

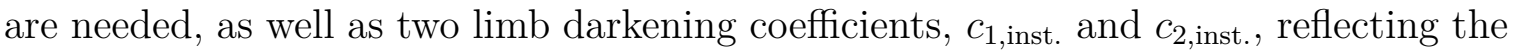
wavelength response of the optical setup of each telescope per instrument. Per planet $p$ (element of the planets designated letters) the following parameters are needed:

- A mass ratio, where for the innermost planet the ratio to the central star $m_{p} / m_{S}$ is used and for all others the ratio to the next inner planet $m_{p} / m_{p^{\prime}}$.

- The semi-major axis $a_{p}$ calculated from the earlier derived mean period $P_{p}$, and as a free parameter a correction factor, $a_{p \text {,corr }}$, that gives the deviance to a pure Keplerian orbit:

$$
a_{p}=\left(\frac{P_{p}^{2} G\left(m_{S}+m_{p}\right)}{4 \pi^{2}}\right)^{1 / 3} \cdot a_{p, \mathrm{corr}}
$$


with the gravitational constant $G$. For non-transiting planets the semi-major axis is computed from its period given by a period ratio to the next inner planet, $P_{p} / P_{p^{\prime}}$

- The eccentricities of the planets, $e_{p}$.

- The orbital angles: inclination $i_{p}$, argument of periastron $\omega_{p}$, and the longitude of the ascending node $\Omega_{p}$. The latter is fixed to zero for the innermost planet due to the fact of the impossibility to determine the orbits position on the plane of sky. The relative positions of the orbits between the planets is allocatable in the data. Therefore, the longitude of the ascending node of all other planets are modelled and reflect the difference to the innermost planet.

- The position of the planets at the given reference time via the mean anomaly $M_{p}$. It is calculated from a Keplerian orbit and corrected with an addition, $M_{p, \text { corr }}$, that delivers the difference to the Keplerian orbit due to the gravitational interaction between the planets:

$$
M_{p}=M_{p, \text { Kepler }}-\frac{2 \pi}{P_{p}} \Delta T_{p, 0}+M_{p, \text { corr }}
$$

The Keplerian orbit mean anomaly $M_{p, \text { Kepler }}$, is calculated for the transit time from the argument of periastron $\omega_{p}$, and the eccentricity $e_{p}$. This value is extrapolated to the reference time with the mean period, $P_{p}$ and the offset, $\Delta T_{p, 0}$.

- The planet-to-star radius ratio $R_{p} / R_{S}$ for transiting planets.

To explore the parameter space the photodynamical model is coupled to the Markov chain Monte Carlo algorithm emcee Foreman-Mackey et al. (2013). The fit parameters have uniform priors with large limits for the sole purpose of avoiding non-physical results.

\subsection{This thesis}

Not all of the many discovered exoplanets are very well characterised, which would be helpful for understanding planetary system architectures and formation scenarios. The KOINet project is dedicated on improving the lack of information about the systems for exoplanets detected with the Kepler telescope, showing transit timing variations and feasible for ground-based follow-up observations. Anchored in this project, this thesis is a contribution to the field with detailed characterisations of two different KOINet systems by carrying out follow-up observations of the planetary transits and system analyse using the photodynamical model described. As a showcase, the Kepler-9 system was chosen (chapter 2). The system contains three confirmed planets with a small inner planet (Kepler-9d) dynamical independent from two outer Neptune-like planets (Kepler-9b and c) near a 2:1 period commensurability 
and mutual interaction observable in anti-correlated strong TTVs of the order of a day. The use of Kepler short cadence data and 13 new transit observation obtained in the framework of the KOINet in a photodynamical analysis of the Kepler-9b/c system lead to a very refined system model with parameter errorbars of the order of $1 \%$ and the prediction that the Kepler-9c transits will disappear around 2050 due to mutual inclination changes. The second target for KOINet observations and photodynamical analysis was the Kepler-82 system (chapter 3). Four confirmed planets are orbiting this host star, with the two inner ones (Kepler-82d and e) dynamically inactive and independent of the two outer ones. Similar to Kepler-9, the two outer planets (Kepler$82 \mathrm{~b}$ and c) are Neptune-like planets near a 2:1 resonance. The TTVs are of the order of hours and - in contrast to Kepler-9b/c - not anti-correlated. Additionally, the TTVs of Kepler-82c show a 3-orbits period chopping component on top of the large period TTVs. This feature hints on another outer planet. Adding KOINet observations to the Kepler measurements lead to the detection of a fifth non-transiting planet (Kepler-82f) near 3:2 period commensurability to Kepler-82c. The two projects will be summarised in chapter 4 


\section{Chapter 2}

\section{Kepler-9}

The following section has been published in Astrophysics \& Astronomy as the article 'Kepler Object of Interest Network II. Photodynamical modelling of Kepler-9 over 8 years of transit observations' (Freudenthal et al., 2018); Credit: J. Freudenthal et al., A\&A, 618, A41, 2018, reproduced with permission (C) ESO.

The publication was leaded in all stages by J. Freudenthal. The text was mainly written by J. Freudenthal. Section 1 was written in cooperation with C. von Essen and S. Dreizler. Section 2 was written by C. von Essen. Text editing was done by all co-authors and a language editor assigned by A\&A. The KOINet data used in the paper were provided by all authors except J. Freudenthal and S. Dreizler. The KOINet data reduction and a first single transit analysis was conducted by C. von Essen in cooperation with J. Freudenthal. The photodynamical analysis and its interpretation was performed by J. Freudenthal under supervision of C. von Essen and S. Dreizler.

\subsection{Paper: Kepler Object of Interest Network II. Photodynamical modelling of Kepler-9 over 8 years of transit observations}




\title{
Kepler Object of Interest Network ${ }^{\star}$
}

\section{Photodynamical modelling of Kepler-9 over 8 years of transit observations}

\author{
J. Freudenthal ${ }^{1}$, C. von Essen ${ }^{2,1}$, S. Dreizler ${ }^{1}$, S. Wedemeyer ${ }^{3,4}$, E. Agol ${ }^{5,6,7, b}$, B. M. Morris ${ }^{5}$, A. C. Becker ${ }^{5}$,

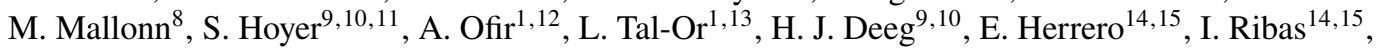 \\ S. Khalafinejad ${ }^{16,17}$, J. Hernández ${ }^{18}$, and M. M. Rodríguez S. ${ }^{18}$ \\ ${ }^{1}$ Institut für Astrophysik, Georg-August-Universität Göttingen, Friedrich-Hund-Platz, 1, 37077 Göttingen, Germany \\ e-mail: jfreude@astro.physik.uni-goettingen.de \\ 2 Stellar Astrophysics Centre, Aarhus University, Ny Munkegade 120, 8000 Aarhus, Denmark \\ 3 Rosseland Centre for Solar Physics, University of Oslo, PO Box 1029 Blindern, 0315 Oslo, Norway \\ ${ }^{4}$ Institute of Theoretical Astrophysics, University of Oslo, PO Box 1029 Blindern, 0315 Oslo, Norway \\ 5 Astronomy Department, University of Washington, Seattle, WA 98195, USA \\ ${ }^{6}$ Institut d'Astrophysique de Paris, 98 bis Boulevard Arago, 75014 Paris, France \\ 7 Virtual Planetary Laboratory, University of Washington, USA \\ ${ }^{8}$ Leibniz-Institut für Astrophysik Potsdam, An der Sternwarte 16, 14482 Potsdam, Germany \\ ${ }^{9}$ Instituto de Astrofísica de Canarias, C. Vía Láctea s/n, 38205 La Laguna, Tenerife, Spain \\ ${ }^{10}$ Universidad de La Laguna, Departamento de Astrofísica, 38206 La Laguna, Tenerife, Spain \\ 11 Aix-Marseille Université, CNRS, LAM, Laboratoire d'Astrophysique de Marseille, Marseille, France \\ ${ }^{12}$ Department of Earth and Planetary Sciences, Weizmann Institute of Science, Rehovot, 76100, Israel \\ ${ }_{13}$ School of Geosciences, Raymond and Beverly Sackler Faculty of Exact Sciences, Tel Aviv University, Tel Aviv, 6997801, Israel \\ ${ }^{14}$ Institut de Cincies de l'Espai (IEEC-CSIC), C. Can Magrans s/n, Campus UAB, 08193 Bellaterra, Spain \\ ${ }^{15}$ Institut Estudis Espacials de Catalunya (IEEC), C. Gran Capità 204, Edif. Nexus, 08034 Barcelona, Spain \\ ${ }^{16}$ Hamburg Observatory, Hamburg University, Gojenbergsweg 112, 21029 Hamburg, Germany \\ ${ }_{17}$ Max Planck Institute for Astronomy, Königstuhl 17, 69117 Heidelberg, Germany \\ ${ }^{18}$ Instituto de Astronomía, UNAM, Campus Ensenada, Carretera Tijuana-Ensenada km 103, 22860 Ensenada, México
}

Received 16 May 2018 / Accepted 28 June 2018

\section{ABSTRACT}

Context. The Kepler Object of Interest Network (KOINet) is a multi-site network of telescopes around the globe organised to follow up transiting planet-candidate Kepler objects of interest (KOIs) with large transit timing variations (TTVs). Its main goal is to complete their TTV curves, as the Kepler telescope no longer observes the original Kepler field.

Aims. Combining Kepler and new ground-based transit data we improve the modelling of these systems. To this end, we have developed a photodynamical model, and we demonstrate its performance using the Kepler-9 system as an example.

Methods. Our comprehensive analysis combines the numerical integration of the system's dynamics over the time span of the observations along with the transit light curve model. This provides a coherent description of all observations simultaneously. This model is coupled with a Markov chain Monte Carlo algorithm, allowing for the exploration of the model parameter space.

Results. Applied to the Kepler-9 long cadence data, short cadence data, and 13 new transit observations collected by KOINet between the years 2014 and 2017, our modelling provides well constrained predictions for the next transits and the system's parameters. We have determined the densities of the planets Kepler-9b and $9 \mathrm{c}$ to the very precise values of $\rho_{b}=0.439 \pm 0.023 \mathrm{~g} \mathrm{~cm}{ }^{-3}$ and $\rho_{c}=0.322 \pm 0.017 \mathrm{~g} \mathrm{~cm}^{-3}$. Our analysis reveals that Kepler-9c will stop transiting in about $30 \mathrm{yr}$ due to strong dynamical interactions between Kepler-9b and 9c, near 2:1 resonance, leading to a periodic change in inclination.

Conclusions. Over the next 30 years, the inclination of Kepler-9c (-9b) will decrease (increase) slowly. This should be measurable by a substantial decrease (increase) in the transit duration, in as soon as a few years' time. Observations that contradict this prediction might indicate the presence of additional objects in this system. If this prediction turns out to be accurate, this behaviour opens up a unique chance to scan the different latitudes of a star: high latitudes with planet $\mathrm{c}$ and low latitudes with planet $\mathrm{b}$.

Key words. planetary systems - planets and satellites: dynamical evolution and stability - methods: data analysis techniques: photometric - stars: individual: Kepler-9 - stars: fundamental parameters

\section{Introduction}

One of the outstanding results of the Kepler mission (Borucki et al. 2010) is the large number of transiting multi-planet systems.

\footnotetext{
${ }^{\star}$ Ground-based photometry is only available at the CDS via anonymous ftp to cdsarc.u-strasbg.fr $(130.79 .128 .5)$ or via http: //cdsarc.u-strasbg.fr/viz-bin/qcat?J/A+A/618/A41

${ }^{\mathrm{b}}$ Guggenheim Fellow.
}

Prior to Kepler's launch, it was shown that the analysis of the dynamical interaction in multi-planet systems would be feasible offering an independent mass determination (Holman \& Murray 2005; Agol et al. 2005). This was impressively confirmed from the first multi-transiting systems (Holman et al. 2010; Lissauer et al. 2011a) using transit timing variations (TTVs), that is, deviations from strict periodicity in planetary transits, caused by nonKeplerian forces. The first compilation of such systems revealed 
that multi-planet systems are preferentially found among lowermass planets (Latham et al. 2011) highlighting the advantages of TTVs over radial velocity measurements. Since Kepler, the search for transiting multi-planet systems has revealed objects such as TRAPPIST-1 (Gillon 2016), with three potentially habitable rocky planets, Kepler-80, a resonant chain of five planets, and Kepler-90, the first eight-planet system (Shallue \& Vanderburg 2018).

Transiting multi-planet systems close to resonance allow for the determination of planetary radii and masses - and therefore bulk densities - from transit light curves alone, which has been intensively explored by Lissauer et al. (2011b), Jontof-Hutter et al. (2016), and Hadden \& Lithwick (2017). A comparison between the two independent mass determinations, namely using radial velocity and transit timing variations, allows for the investigation of systematic errors due to observational and methodological biases (Mills \& Mazeh 2017).

In order to tap into the dynamical information of TTVs it is important to cover a full cycle of orbital momentum and energy exchange between the planets (henceforth "interaction cycle"), which can be substantially longer than their orbital periods. One of the first lists of systems showing TTVs (Mazeh et al. 2013) revealed the large existing fraction of Kepler objects of interest (KOIs) that could not be used for dynamical analysis due to long interaction cycles. These were longer than, or of the order of, Kepler's total observing time. This motivated us to create and coordinate the Kepler Object of Interest Network, (KOINet ${ }^{1}$, von Essen et al. 2018), a network of ground-based telescopes organised to follow up KOIs with large-amplitude TTVs. The main goal of KOINet is to coordinate already existing telescopes to characterise the masses of planets and planetary candidates by analysing their observed transit timing variations.

Among the KOINet targets, Kepler-9 is a benchmark system. The star is a solar analog and two of its planets are close to a 2:1 mean motion resonance, with TTV amplitudes of the order of one day. Their deep transits $(\sim 0.5 \%)$ combined with their large interaction times and the magnitude of the host star $\left(\mathrm{K}_{P}=13.803\right)$ make this system ideal for photometric groundbased follow-up studies.

The first TTV analysis of the Kepler-9b/c system with an incomplete coverage of the interaction cycle had to be complemented with (a few) radial velocity measurements (Holman et al. 2010) which resulted in Saturn-mass planets. The composition of these two planets was investigated by Havel et al. (2011) from evolutionary models, as well as the stellar mass and radius. Using most or all long-cadence Kepler data, several authors revised the planetary masses from TTVs alone (Borsato et al. 2014; Dreizler \& Ofir 2014) finding masses of about half the values previously reported in the first paper. Dreizler \& Ofir (2014) thereby showed that the confirmed innermost planet, Kepler-9d, is dynamically independent from this near-resonant pair. Recently, a new transit observation for Kepler-9b (Wang et al. 2018b) was used to correct its transit time predictions. Additionally, the observation of the Rossiter-McLaughlin effect in radial velocity measurements of Kepler-9 (Wang et al. 2018a) indicates that the stellar spin axis is very likely aligned with the planetary orbital plane.

In this paper, we exploit the large amount of short-cadence Kepler data, complemented by long-cadence Kepler data where short-cadence observations are missing, and extended three years in time by adding corresponding ground-based light curves

1 http://koinet.astro.physik.uni-goettingen.de from KOINet, all wrapped in a detailed photodynamical analysis. The observation of the full interaction cycle by the KOINet follow-up together with the comprehensive analysis results in Kepler-9b and $9 \mathrm{c}$ being the system with the highest-precision planetary mass and radius determinations. We also constrain the stellar parameters of the host star and predict the dynamical evolution of the system for the next few decades.

This paper is divided as follows. We describe the new transit observations by the KOINet, their reduction and analysis in Sect. 2. The structure of the photodynamical model used to analyse KOINet systems is described in Sect. 3. A description of the results from this analysis for the Kepler-9 system can be found in Sect. 4 and these results are discussed in Sect. 5. We end the paper with some conclusions in Sect. 6.

\section{Observations, data reduction, and analysis}

Between June, 2014, and September, 2017, we observed 13 primary transits of the Kepler-9b/c planets. The photometric follow-up was carried out in the framework of KOINet (von Essen et al. 2018). In this work, we combine the Kepler photometry with new ground-based data which have been collected after the nominal time of the Kepler Space Telescope. This section covers the treatment of the new ground-based observations. The photodynamical model described in Sect. 3 was previously fitted to the available Kepler data with the aim of obtaining initial parameters for the ground-based data analysis. A description of the photodynamical analysis on the different data sets follows in Sect. 4.

\subsection{Data acquisition and main characteristics of the collected photometry}

Table 1 shows the main characteristics of the data presented in this work. These are the date in which the observations were performed, in years, months, and days; the planet observed during transit; an acronym for the telescope used to carry out the observations; the precision of the data in parts-per-thousand (ppt); the number of frames collected during the night, $N$; the cadence of the data accounting for readout time in seconds, CAD; the total duration of the observations in hours, $T_{\text {tot }}$; and the transit coverage, TC. To increase the photometric precision of the collected data, when possible we slightly defocused the telescopes (Kjeldsen \& Frandsen 1992; Southworth et al. 2009).

Below is a brief description of the main characteristics of each of the telescopes involved in this work.

The Oskar Lühning Telescope (OLT $1.2 \mathrm{~m}$ ) has a $1.2 \mathrm{~m}$ aperture diameter and is located at the Hamburger Observatory in Hamburg, Germany. The telescope can be used remotely and has a guiding system, minimising systematics caused by poor tracking. Although the seeing at the observatory is relatively poor (typical values are around 3-4 arcsec), it remains constant during the night, allowing photometric precision in the ppt level. Here we analyse one light curve taken during our first observing season.

The Apache Point Observatory hosts the Astrophysical Research Consortium $3.5 \mathrm{~m}$ telescope (henceforth "ARC $3.5 \mathrm{~m}$ "), and is located in New Mexico, in the United States of America. Due to the large collecting area, typically 2000 observations per observing run were collected with this telescope. For our observations, the telescope was slightly defocused. The photodynamical analysis of Kepler-9 presented here includes three light curves taken with the ARC $3.5 \mathrm{~m}$ during our second observing campaign in 2015. 
J. Freudenthal et al.: Kepler Object of Interest Network. II.

Table 1. Characteristics of the collected ground-based transit light curves of Kepler-9b/c, collected in the framework of KOINet.

\begin{tabular}{|c|c|c|c|c|c|c|c|}
\hline $\begin{array}{c}\text { Date } \\
\text { (yyyy.mm.dd) }\end{array}$ & Planet & Telescope & $\begin{array}{c}\sigma_{\text {res }} \\
(\mathrm{ppt})\end{array}$ & $\mathrm{N}$ & $\begin{array}{l}\text { CAD } \\
(\mathrm{s})\end{array}$ & $\begin{array}{c}T_{\text {tot }} \\
\text { (hours) }\end{array}$ & $\mathrm{TC}$ \\
\hline 2014.06.30 & $\mathrm{c}$ & OLT $1.2 \mathrm{~m}$ & 3.6 & 103 & 79 & 2.3 & - - - E O \\
\hline 2015.06 .17 & $\mathrm{~b}$ & ARC $3.5 \mathrm{~m}$ & 1.9 & 2075 & 8 & 4.7 & O I B - - \\
\hline \multirow[t]{4}{*}{2015.07 .25} & $\mathrm{~b}$ & WISE $1 \mathrm{~m}$ & 2.2 & 132 & 166 & 6.1 & O I B E - \\
\hline & $\mathrm{b}$ & CAHA $2.2 \mathrm{~m}$ & 1.6 & 462 & 57 & 7.4 & O I B E O \\
\hline & $b$ & LIV 2 m & 1.2 & 545 & 46 & 7.1 & - - B E O \\
\hline & $\mathrm{b}$ & NOT $2.5 \mathrm{~m}$ & 1.5 & 630 & 28 & 5.0 & - - B E O \\
\hline 2015.08 .14 & $\mathrm{~b}$ & ARC $3.5 \mathrm{~m}$ & 2.7 & 2095 & 7 & 3.8 & - I B E - \\
\hline 2015.09 .01 & $\mathrm{c}$ & ARC $3.5 \mathrm{~m}$ & 2.2 & 2073 & 4 & 2.5 & - - B E O \\
\hline \multirow[t]{2}{*}{2015.10 .10} & $\mathrm{~b}$ & IAC $0.8 \mathrm{~m}$ & 0.5 & 60 & 197 & 3.3 & - I B - - \\
\hline & $\mathrm{b}$ & TJO $0.8 \mathrm{~m}$ & 1.8 & 133 & 61 & 2.3 & - I B - - \\
\hline 2017.05 .17 & $\mathrm{c}$ & NOT $2.5 \mathrm{~m}$ & 0.8 & 219 & 79 & 4.9 & O I B E - \\
\hline 2017.06 .16 & $\mathrm{~b}$ & NOT $2.5 \mathrm{~m}$ & 1.1 & 624 & 30 & 5.4 & - I B E O \\
\hline 2017.06 .25 & $\mathrm{c}$ & NOT $2.5 \mathrm{~m}$ & 1.2 & 416 & 42 & 4.9 & O I B - - \\
\hline 2014.06 .27 & & OANLH $1 \mathrm{~m}$ & \multicolumn{5}{|c|}{ Technical difficulties } \\
\hline 2014.07 .23 & & OLT $1.2 \mathrm{~m}$ & \multicolumn{5}{|c|}{ Technical difficulties } \\
\hline \multirow[t]{2}{*}{2014.08 .12} & & OLT $1.2 \mathrm{~m}$ & \multicolumn{5}{|c|}{ Weather problems } \\
\hline & & LIV 2 m & \multicolumn{5}{|c|}{ Weather problems } \\
\hline 2015.03 .13 & & OLT $1.2 \mathrm{~m}$ & \multicolumn{5}{|c|}{ Weather problems } \\
\hline 2016.05 .10 & & ARC $3.5 \mathrm{~m}$ & \multicolumn{5}{|c|}{ Technical difficulties } \\
\hline 2017.09.01 & & NOT $2.5 \mathrm{~m}$ & \multicolumn{5}{|c|}{ Weather problems } \\
\hline
\end{tabular}

Notes. The letter code to specify the transit coverage during each observation is the following: O: out of transit, before ingress. I: ingress. B: flat bottom. E: egress. O: out of transit, after egress.

The Wise Observatory hosts a $1 \mathrm{~m}$ telescope that is operated by Tel Aviv University, Israel (WISE $1 \mathrm{~m}$ ). Here we present one transit taken during the second campaign in 2015.

The Centro Astronómico Hispano-Alemán hosts, among others, a $2.2 \mathrm{~m}$ telescope (henceforth "CAHA $2.2 \mathrm{~m}$ "). Here we present one complete transit observation of Kepler-9b.

The fully robotic $2 \mathrm{~m}$ Liverpool telescope (LIV $2 \mathrm{~m}$; Steele et al. 2004) is located at the Observatorio Roque de los Muchachos and is owned and operated by Liverpool John Moores University. In this work, we present one transit observation taken with LIV $2 \mathrm{~m}$ during our second observing season.

The $2.5 \mathrm{~m}$ Nordic Optical Telescope (NOT $2.5 \mathrm{~m}$ ) is located at the Observatorio Roque de los Muchachos in La Palma, Spain. Currently, telescope time for KOINet is assigned via a large ( 3 yr) program. Here, we analyse four light curves taken between the first and fourth observing seasons.

The 80 centimetre telescope of the Instituto de Astrofísica de Canarias (IAC $0.8 \mathrm{~m}$ ) is located at the Observatorio del Teide, in the Canary Islands, Spain. Observations were collected by KOINet's observers on site. Here we present one light curve taken during our second observing season.

The Telescopi Joan Oró (TJO) is a fully robotic 80 centimetre telescope located at the Observatori Astronomic del Montsec, in the north-east of Spain (henceforth "TJO $0.8 \mathrm{~m}$ "). Here we present one transit light curve.

The Observatorio Astronómico Nacional Llano del Hato, Venezuela, hosts a $1 \mathrm{~m}$ Zeiss reflector (henceforth "OANLH $1 \mathrm{~m}$ "). During scheduled observations, the telescope suffered from technical difficulties.

\subsection{Data reduction and preparation}

To reduce the impact of Earth's atmosphere and the associated telluric contamination in the $I$-band, as well as the absorption of stellar light at shorter wavelengths, all of our observations are carried out using an $R$-band filter. Observers always provide a set of calibration frames (bias and flat fields) that are used to carry out the photometric data reduction. To reduce the data and construct the photometric light curves, we use our own IRAF and python-based pipelines called Differential Photometry Pipelines for Optimum light curves, DIP ${ }^{2} \mathrm{OL}$. A full description of DIP ${ }^{2} \mathrm{OL}$ can be found in von Essen et al. (2018). Briefly, the IRAF component of DIP ${ }^{2} \mathrm{OL}$ measures fluxes for different reference stars, apertures, and sky rings; the latter two are set in proportion to the intra-night averaged full width at half maximum (FWHM). The python counterpart of $\mathrm{DIP}^{2} \mathrm{OL}$ finds the optimum combination of reference stars, aperture, and width of the sky ring that minimises the scatter of the photometric light curves. Once the light curves are constructed, we transform the time stamps from Universal Time to Barycentric Julian Dates in Barycentric Dynamical Time ( $\mathrm{BJD}_{\mathrm{TDB}}$ ) using Eastman et al. (2010)'s web tool, all wrapped up in a python script.

To detrend the light curves, we compute the time-dependent $x$ and $y$ centroid positions of all the stars, the background counts from the sky rings, the integrated flat counts for the final aperture centered around the centroid positions, the airmass, and the seeing, all from the photometric science frames. A full description of our detrending strategy, how we combine these quantities to construct the detrending function, and the extra care in the particular choice and number of detrending parameters can be found in Sect. 4.2 of von Essen et al. (2018). The detrending components, and the time, flux, and errors, are injected into the transit fitting routine.

\subsection{First data analysis}

Before fitting the full data set using our photodynamical code (see Sect. 3) we carry out a transit fit to each ground-based light 
curve individually. The main goal of this step is to provide accurate error bars for the flux measurements, that are enlarged to account for correlated noise (see e.g. Carter \& Winn 2009). A detailed description of the transit-fitting procedure can be found in Sect. 4 of von Essen et al. (2018). Briefly, once the detrending components are selected, we fit each transit light curve individually. For this, we use a detrending times transit (Mandel \& Agol 2002) model, with a quadratic limb-darkening law and hence, quadratic limb-darkening coefficients. The latter are computed as described in von Essen et al. (2013), for stellar parameters closely matching the ones of Kepler-9 (Holman et al. 2010) and a Johnson-Cousins R filter transmission response. As initial values for all the transit parameters, we use the ones given by the photodynamical analysis carried out on Kepler data only. Since the TTVs in this system are so large, all of the transit parameters have to be computed for each specific light curve. When fitting for the transit parameters, rather than using uniform distributions for these parameters, we use Gaussian priors with mean and standard deviation equal to the values computed from our initial photodynamical analysis on Kepler data. Only when the transit is fully observed do we allow the model to fit for the semi-major axis, the inclination, and the planet-to-star radius ratio, along with the mid-transit time. Otherwise, all of the transit parameters remain fixed and we fit for the mid-transit time only.

To determine reliable errors for the fitted parameters, we compute them from posterior-probability distributions using a Markov chain Monte Carlo (MCMC) approach. At this stage, we iterate 100000 times per transit, and discard a conservative first $20 \%$. Once the best-fit parameters are obtained, we compute residual light curves by subtracting from the data our best-fit transit-times-detrending models. From the residuals we compute the $\beta$ factor as fully described in Sect. 4.2 of von Essen et al. (2018). Here, we average $\beta$ values computed in time bins of 0.8 , $0.9,1,1.1$, and 1.2 times the duration of ingress. If this averaged $\beta$ factor is larger than 1 , we enlarge the photometric error bars by this value, and we repeat the MCMC fitting in exactly the same fashion as previously explained. The raw light curves obtained after the second MCMC iteration with their error bars enlarged, along with the number of detrending components per light curve, are the input parameters of the photodynamical analysis. As a consistency check, after the photodynamical analysis is complete, we compare the derived detrending coefficients to the ones obtained from individually fitting the light curves.

\subsection{Independent check of the timings}

The use of KOINet to carry out TTV studies relates observations taken with several telescopes. As a consequence, the timings will be subject to the accuracy of the ground-based observatories, and the success of KOINet will rely on the capabilities of the many observatories involved in our photometric follow-up to accurately record timings.

In order to investigate this, on the night of July 25, 2015, we observed Kepler-9b using four different telescopes, namely CAHA $2.2 \mathrm{~m}$, LIV $2 \mathrm{~m}$, WISE $1 \mathrm{~m}$, and NOT $2.5 \mathrm{~m}$. Only in the case of CAHA $2.2 \mathrm{~m}$ did we have full transit coverage. After fitting for the transit parameters as previously specified, we obtained in this case the semi-major axis, $a / R_{S}$, the inclination, $i$, the planet-to-star radii ratio, $R_{P} / R_{S}$, and the mid-transit time, $T_{0}$. The derived values along with their $1-\sigma$ uncertainties can be found in the top part of Table 2. Within errors, all the fitted parameters are consistent with the values predicted by our photodynamical analysis. The bottom part of the same table shows
Table 2. Transit parameters obtained fitting one light curve of Kepler-9b observed with CAHA $2.2 \mathrm{~m}$ on the night of 2015.07.25.

\begin{tabular}{lcc}
\hline \hline TP, CAHA $2.2 \mathrm{~m}$ & TFP & PDA \\
\hline$a / R_{S}$ & $29.3 \pm 0.2$ & $29.27 \pm 0.1$ \\
$i\left[^{\circ}\right]$ & $88.77 \pm 0.03$ & $88.76 \pm 0.2$ \\
$R_{P} / R_{S}$ & $0.085 \pm 0.001$ & $0.079 \pm 0.003$ \\
\hline Telescope & $T_{0} \pm 1-\sigma$ & \\
\hline CAHA $2.2 \mathrm{~m}$, & $229.4598 \pm 0.0008$ & $229.4606 \pm 0.005$ \\
LIV $2 \mathrm{~m}$ & $229.4629 \pm 0.0009$ & \\
WISE $1 \mathrm{~m}$ & $229.4623 \pm 0.0026$ & \\
NOT $2.5 \mathrm{~m}$ & $229.4582 \pm 0.0018$ & \\
\hline
\end{tabular}

Notes. From left to right (top): transit parameter (TP), transit fitted parameter (TFP) along with 1- $\sigma$ uncertainties, and value predicted by our photodynamical analysis (PDA) applied at this stage to Kepler data only. Bottom: mid-transit times obtained fitting the three remaining incomplete transit light curves. The first column shows the acronym for the telescope, and the second column the timings along with 1- $\sigma$ uncertainties. $T_{0}$ 's are given in $\mathrm{BJD}_{\mathrm{TDB}}-2457000$.

the individual mid-transit times obtained from fitting all the transit parameters for CAHA $2.2 \mathrm{~m}$, and fixing all values except the mid-transit times for the remaining three. All mid-transit times are mutually consistent.

Figure 1 shows the quality of our reduction and analysis procedure. From top to bottom, we show the light curves of Kepler-9b obtained with CAHA $2.2 \mathrm{~m}$ in filled circles, LIV $2 \mathrm{~m}$ in empty squares, WISE $1 \mathrm{~m}$ in empty polygons, and NOT $2.5 \mathrm{~m}$ in filled diamonds. The light curves have been shifted to the predicted mid-transit time. Visual inspection confirms the equivalency of the derived mid-transit times. The consistency among mid-transit times alleviates the uncertainty that exists when using different sites to follow-up one target.

\section{The photodynamical model}

With the aim of producing a tool to determine planetary masses that is applicable to all of our KOINet objects, we developed a photodynamical model. Our light curve analysis is based on an n-body simulation of the planetary system over the time span of the observations, combined with a transit light curve model. The numerical integration is implemented in the Mercury6 package by Chambers (1999). We use the second-order mixed-variable symplectic (MVS) algorithm of the package, which is faster than the Bulirsch-Stoer (BS) algorithm but still applicable. The BS integrator would offer two advantages: the possibility of simulating close encounters and the precision in high-frequency terms of the Hamiltonian (discussed by Deck et al. 2014). The former is insignificant as the Kepler-9b/c system does not perform close encounters. The latter was tested to be negligible in our analysis. We calculated the difference of the same TTV model derived with the MVS integrator and the BS algorithm. Within a 50-yr integration, the difference shows a small slope, which can be corrected by a small change in the mean period smaller than $0.5 \mathrm{~s}$ and an oscillation with increasing amplitude. The amplitude of the oscillation is at most (in these $50 \mathrm{yr}$ ) of the order of $55 \mathrm{~s}$ which is of the order of the precision in the TTVs. For the 8 yr of Kepler- 9 observations, the MVS integrator has sufficient precision. We added a first-order post-Newtonian correction (Kidder 1995) and wrote a python-wrapper for Mercury6 (Husser, priv. comm.). From the n-body simulation, we extract the projected distance between planets and star centres, that 


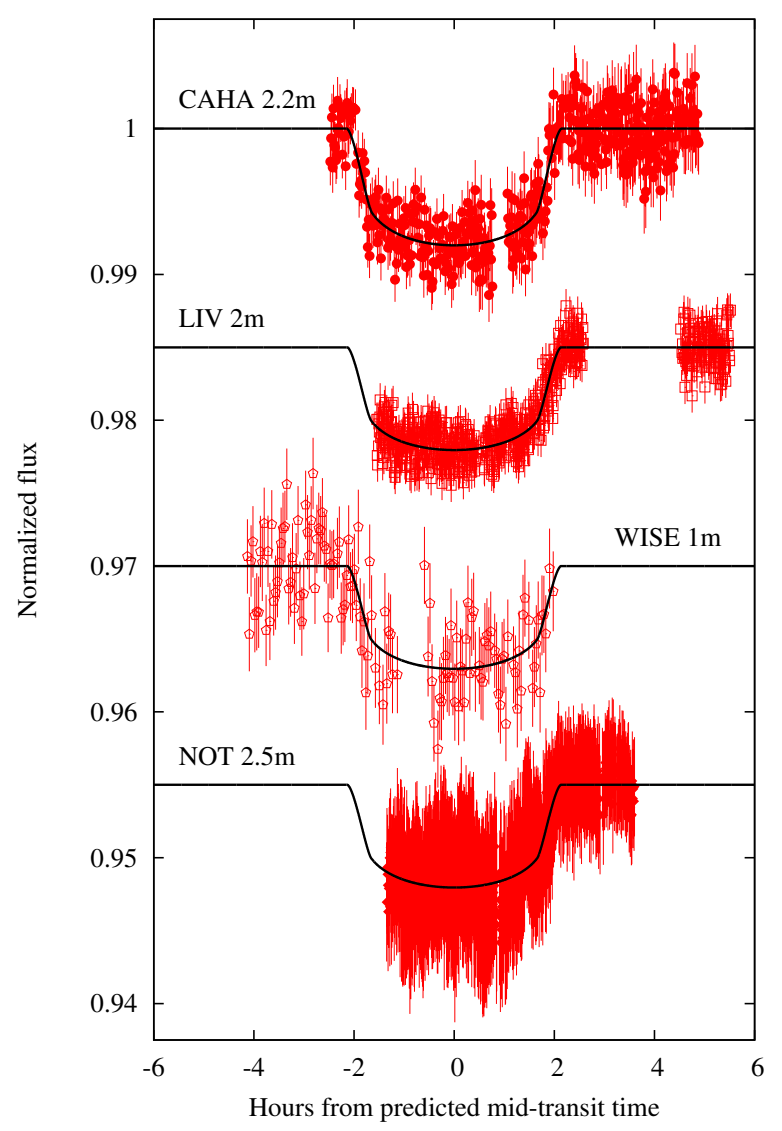

Fig. 1. Detrended transits of Kepler-9b observed on July 25, 2015, by four different telescopes. The transits are artificially shifted for better visual inspection, and plotted as a function of hours from the predicted mid-transit time to appreciate the duration of the observations. Each light curve has been labelled according to the corresponding telescope.

is in turn used to calculate the transit light curve through the Mandel \& Agol (2002) model. Here we use a quadratic limbdarkening law already implemented in the occultquad routine. Finally, for each individual planets, we correct the output time by the light-travel-time effect.

As the numerical integration and its processing are computationally time consuming, we first carry out a coarse integration with a step size equal to only a twentieth of the period of the innermost simulated planet. A more detailed integration is produced for the parts where transits take place and where data are available. For these cases, the detailed integration is done with a step size of 0.01 days, which corresponds to a light curve accuracy of $0.01 \mathrm{ppm}$ for long-cadence Kepler data. This accuracy is measured by the mean difference of transit light curves between a model with the given step size and a model with half the step size. For transit light curves, a time step comparable to ingress/egress duration would have a significant impact on the derivation of the transit parameters (Kipping 2010). Therefore, we calculate the transit model on a fine grid $(\sim 1 \mathrm{~min}$, when needed) and we rebin this to the actual data points. We describe this in more detail in Sect. 4. For our model calculations, we define the $x-y$ plane as the plane of the sky, with its origin placed at the stellar centre. Therefore, these coordinates coincide with the projected distances between planet and star mid points. The positive $z$-axis corresponds to the line of sight, so that the planets transit with positive $z$-values. The sampling of the Mercury6 integration does not match the observation times. To interpolate the projected distances from the Mercury6 results, we model them with a hyperbola in the range of a transit. The Mandel \& Agol (2002) model is calculated for the observation points by these interpolated projected distances, quadratic limb darkening coefficients, and the planet-star radii ratios.

To explore the parameter space, our model is coupled to the MCMC emcee algorithm (Foreman-Mackey et al. 2013) accessible in the PyAstronomy ${ }^{2}$ library. All fitting parameters have uniform priors with large limits with the sole purpose of avoiding non-physical results. Our choice of free parameters is guided by the modelling rather than by the observations. For instance, Mercury 6 uses the semi-major axis, $a$, of the planet as input value. Instead of the period, $P$, we therefore use a correction factor to a mean semi-major axis $a_{\text {adjust }}$ as a free parameter. The mean semi-major axis is calculated through Kepler's third law from the mean period of the transit times of the planets. In addition, the mean anomaly, $M$, is calculated from this mean period, as well as the reference time, $\Delta T_{0}$. As a free parameter, we have an addend to this derived mean anomaly $M_{\text {adjust }}$. Furthermore, Mercury 6 uses the eccentricity, $e$, and the three angles that describe the position of the orbits on the sky. They are the orbital inclination, $i$, the argument of the periastron, $\omega$, and the longitude of the ascending node, $\Omega$. As the orientation in the plane of the sky is not directly measurable, $\Omega$ is fixed to zero for the innermost simulated planet. In this way, the corresponding values of the other planets show the difference in comparison to this first one. Last but not least, Mercury6 requires the masses, $m$, of the central star and the planets. These are given by an absolute value for the central star, the ratio of the masses of the innermost simulated planet to the central star, and the ratio of masses of the other planets to the innermost planet.

In order to calculate the transit light curve from the output of Mercury6, the stellar radius, $R_{S}$, is required to calculate the relative planet-star distance normalised to the stellar radius. The transit measurements constrain the stellar density (Agol \& Fabrycky 2017), but we choose to directly use the required model parameters. Instead of the stellar density, we input the stellar mass and radius, but fix one of them during the modelling. In addition, the occultquad routine requires the planet-star radius ratio, $R_{p} / R_{S}$, and the two quadratic limb darkening coefficients, $c_{1}$ and $c_{2}$.

\section{Dynamical analysis of Kepler-9}

Three different approaches were taken to dynamically characterise the Kepler-9b/c system. Firstly, in order to compare the photodynamical model with the dynamical analysis of only transit times, we fitted our model to quarters 1-16 of the Kepler longcadence data (hereafter data set I). This allowed us to compare our results to those given by Dreizler \& Ofir (2014). Secondly, we attempted to constrain the stellar radius by means of Kepler short-cadence data, since they have a sampling rate that is thirty times greater. To this end, we replaced Kepler long-cadence data with short-cadence data when available. Specifically, for Kepler-9, short cadence data are available between quarters 7 and 17 (data set II). Finally, the model is applied to the full data set, which comprises long-cadence data for Kepler quarters $1-6$, short-cadence data covering quarters $7-17$, and all new

2 https://github.com/sczesla/PyAstronomy 
ground-based light curves, 13 in total, that were collected by KOINet (data set III).

The results from all the data analyses, and a comparison to previous analyses, are listed in Table 3. The top part of the table shows the stellar parameters. The literature values of the stellar radius and density parameters are taken from Havel et al. (2011), and the respective quadratic limb darkening values are taken from the NASA Exoplanet Archive (Mullally et al. 2015). The bottom part of Table 3 shows the derived planetary parameters. These are compared to the results given by Dreizler \& Ofir (2014). In this latter work, the authors modelled the individual transits observed in long-cadence data, from where the mid-transit times were derived. Afterwards, they dynamically modelled these transit times.

The osculating orbital elements are given at a reference time, BJD $=2454933.0$. Fitting the transit times found in Dreizler \& Ofir (2014) with a linear time-dependent model we obtained the reference times $\Delta T_{b}=25.26 \mathrm{~d}$ and $\Delta T_{c}=-3.08 \mathrm{~d}$ as intercepts, and the mean periods $P_{b}=19.247 \mathrm{~d}$ and $P_{c}=38.944 \mathrm{~d}$ as slopes The reference times and mean periods are used for the determination of the semi major axis and the mean anomaly for all data sets, as described previously in Sect. 2.

During our photodynamical modelling we chose to fix the stellar mass to its literature value, $m_{S}=1.05 \pm 0.03 M_{\odot}$ (Havel et al. 2011). Derived parameters that depend on this value are the planetary masses, as the model parameters are given with respect to the stellar mass. Therefore, the uncertainties of the derived parameters are increased using error propagation including the uncertainty of the stellar mass, $\sigma_{m_{S}}=0.03 M_{\odot}$. When applied, in Table 3 these parameters are labelled with " $\sigma_{m_{s}}$ prop." The calculated densities of the star and the planets depend on the stellar mass in the same way. The semi-major axes are also affected. These are computed from the mean period through Kepler's third law, which also includes the stellar and planetary masses. As a consequence, this error is also propagated into the uncertainty of the semi-major axis.

A quick comparative look at Table 3 shows how the limb darkening coefficients obtained modelling data set I significantly differ from their literature values. We address this issue in Sect. 5. With this exception, all planetary parameters are in agreement with prior results within $1-\sigma$ errors. The error bars decrease from modelling data set I to III. The reasons for this are given in detail in the following sections.

\subsection{Treatment of the Kepler data}

To prepare Kepler's transit photometry we first extracted three times the transit duration symmetrically around each transit mid point. To account for intrinsic stellar photometric variability we normalised each transit light curve dividing this by a timedependent second-order polynomial fitted to the out-of-transit data points. To obtain the coefficients of the polynomial functions, we used a simple least-squares minimisation routine. As previously mentioned, for long-cadence data, the light curve model is oversampled by a factor of 30 and rebinned to the actual data points. This procedure is not necessary for short-cadence data. The high signal-to-noise ratio (S/N) of Kepler data allowed us to include the quadratic limb darkening coefficients into our model budget.

\subsection{Treatment of ground-based data}

Due to the lower $\mathrm{S} / \mathrm{N}$ of the ground-based data, we fixed the quadratic limb darkening coefficients to values derived from stellar evolution models for the $R$-band filter, which we used for all our observations. For stellar parameters closely matching the ones of Kepler-9, the derived limb darkening coefficients are $c_{1}=0.46$ and $c_{2}=0.17$. The best-matching coefficients of the previously derived detrending components (see Sect. 2.3) for each ground-based observation are calculated as a linear combination at each call of the photodynamical model.

\subsection{Statistical considerations}

We performed the analysis of data set I with 36 walkers each, iterating over 30000 steps. The starting parameters of the walkers are randomly chosen from a normal distribution around the parameter results of Dreizler \& Ofir (2014) with a 3- $\sigma$ width. The walkers needed 2000 iterations to burn in, with the exception of one that finished in a higher $\chi^{2}$ minimum. Therefore, our results are derived from 35 walkers with 28000 iterations each. We calculated the autocorrelation time for each parameter following Goodman \& Weare (2010), but averaging over the autocorrelation function per walker instead of averaging directly over the walker values, as discussed in the Blog by Daniel Foreman-Mackey ${ }^{3}$. These calculations result in an autocorrelation time of 1853 on average (2771 maximum), which gives us an effective sample size of 528 (353 minimum). Each parameter shows a Gaussian posterior distribution from which we extract the median and standard deviation values as best-fit values and errors, respectively. Our results are shown in Table 3. The best-fit solution has a reduced $\chi^{2}$ of 1.48 .

The analysis of data set II is performed using 36 walkers with 20000 iterations each. In this case, they burned in after 4000 iterations, with the exception of two walkers that ended in a higher $\chi^{2}$ minimum. The autocorrelation time averages out at 927 (1648 maximum), which gives an effective sample size of 586 (330 minimum). The resulting parameters are derived using the median and standard deviation of the posterior Gaussian distribution. The best solution of this analysis has a reduced $\chi^{2}$ of 1.06 .

The modelling of data set III is accomplished by 36 walkers with 20000 iterations each. Thirty-five of the walkers burned in after 2000 iterations. The resulting Gaussian distributions of the 630000 iterations for the parameters and their correlations can be seen in Fig. A.7 for the mass-dependent parameters, in Fig. A.8 for the radius-dependent parameters, and in total in Fig. A.9. Our best-fit solution has a reduced $\chi^{2}$ of 0.97 . The autocorrelation length of this analysis is given by a value of 694 on average (1105 maximum). This results in an effective sample size of 907 (570 minimum).

\subsection{Results}

The comparison of the best models to the most recent light curves from 2017 displayed in Fig. 2 clearly shows how the inclusion of our new ground-based light curves leads to an improvement of the derived parameters. The upper plot shows a Kepler-9b transit light curve in red observed on June 16, 2017. The lower plot shows a Kepler-9c transit light curve in blue observed on May 17, 2017. Both light curves were obtained using the NOT $2.5 \mathrm{~m}$ telescope. The variation of 500 randomly chosen good models for data set II is given by the light transparent yellow areas, which can be compared to the corresponding ones obtained including all new ground-based data (data set III). These are plotted in the figures with a light transparent black

\footnotetext{
http://dfm.io/posts/autocorr
} 
J. Freudenthal et al.: Kepler Object of Interest Network. II.

Table 3. Stellar and planetary parameters derived from the photodynamical modelling of data set I in the second column, data set II in the third column, data set III in the fourth column, along with bibliographic values (Dreizler \& Ofir 2014) in the fifth column for comparison and the sixth column displays some parameters corrected by investigating stellar evolution models in Sect. 5.4.

\begin{tabular}{|c|c|c|c|c|c|}
\hline Parameter & Data set I & Data set II & Data set III & Literature & MESA \\
\hline \multicolumn{6}{|l|}{ Stellar parameters } \\
\hline$m_{S}\left(M_{\odot}\right)$ & 1.05(3) (fixed) & 1.05(3) (fixed) & 1.05(3) (fixed) & $1.05(3)$ & $1.04_{-0.04}^{+0.07}$ \\
\hline$R_{S}\left(R_{\odot}\right)$ & $0.947(21)$ & $0.9755(92)$ & $0.9742(83)$ & $1.05(6)$ & $0.971_{-0.021}^{+0.034}$ \\
\hline$\rho_{S}^{*}\left(\mathrm{~g} \mathrm{~cm}^{-3}\right)$ & $1.74(12)$ & $1.596(45)$ & $1.603(41)$ & $1.12(27)$ & \\
\hline$\rho_{S}^{*}\left(\mathrm{~g} \mathrm{~cm}^{-3}\right)\left(\sigma_{m_{S}}\right.$ prop. $)$ & $1.74(13)$ & $1.596(64)$ & $1.603(61)$ & $1.12(27)$ & \\
\hline$c_{1, \text { Kepler }}$ & $0.281(53)$ & $0.361(51)$ & $0.351(47)$ & 0.4089 & \\
\hline$c_{2, \text { Kepler }}$ & $0.410(95)$ & $0.251(78)$ & $0.269(71)$ & 0.2623 & \\
\hline \multicolumn{6}{|l|}{ Planetary parameters } \\
\hline$m_{b} / m_{S}$ & $0.0001271(11)$ & $0.0001271(11)$ & $0.0001281(11)$ & $0.000129(2)$ & \\
\hline$m_{c} / m_{b}$ & $0.68911(26)$ & $0.68846(22)$ & $0.68849(20)$ & $0.6875(3)$ & \\
\hline$m_{b}^{*}\left(M_{\oplus}\right)$ & $44.36(44)$ & $44.51(32)$ & $44.71(24)$ & $45.1(15)$ & \\
\hline$m_{c}^{*}\left(M_{\oplus}\right)$ & $30.57(30)$ & $30.64(22)$ & $30.79(17)$ & 31(1) & \\
\hline$m_{b}^{*}\left(M_{\oplus}\right)\left(\sigma_{m_{s}}\right.$ prop. $)$ & $44.4(13)$ & $44.5(13)$ & $44.7(13)$ & $45.1(15)$ & $44.4_{-1.7}^{+3.0}$ \\
\hline$m_{c}^{*}\left(M_{\oplus}\right)\left(\sigma_{m_{s}}\right.$ prop. $)$ & $30.57(92)$ & $30.64(90)$ & $30.79(90)$ & $31(1)$ & $30.5_{-1.2}^{+2.1}$ \\
\hline$a_{b, \text { adjust }}(\mathrm{AU})$ & $0.9992801(21)$ & $0.9992811(11)$ & $0.9992801(11)$ & - & \\
\hline$a_{c, \text { adjust }}(\mathrm{AU})$ & $1.0015531(31)$ & $1.0015521(31)$ & $1.0015531(21)$ & - & \\
\hline$a_{b}^{*}(\mathrm{AU})$ & $0.14276083(21)$ & $0.14276096(16)$ & $0.14276088(14)$ & $0.143(1)$ & \\
\hline$a_{c}^{*}(\mathrm{AU})$ & $0.22889883(83)$ & $0.22889869(63)$ & $0.22889876(53)$ & $0.229(2)$ & \\
\hline$a_{b}^{*}(\mathrm{AU})\left(\sigma_{m_{S}}\right.$ prop. $)$ & $0.1428(14)$ & $0.1428(14)$ & $0.1428(14)$ & $0.143(1)$ & $0.1423_{-0.0018}^{+0.0032}$ \\
\hline$a_{c}^{*}(\mathrm{AU})\left(\sigma_{m_{S}}\right.$ prop. $)$ & $0.2289(22)$ & $0.2289(22)$ & $0.2289(22)$ & $0.229(2)$ & $0.2282_{-0.0029}^{+0.0051}$ \\
\hline$e_{b}$ & $0.06437(74)$ & $0.06412(54)$ & $0.06378(40)$ & $0.063(1)$ & \\
\hline$e_{c}$ & $0.068026(92)$ & $0.067974(73)$ & $0.067990(68)$ & $0.0684(2)$ & \\
\hline$i_{b}\left(^{\circ}\right)$ & $89.037(85)$ & $88.931(33)$ & $88.936(30)$ & $87.1(7)$ & \\
\hline$i_{c}\left(^{\circ}\right)$ & $89.229(41)$ & $89.177(17)$ & $89.180(15)$ & $87.2(7)$ & \\
\hline$\omega_{b}\left({ }^{\circ}\right)$ & $357.17(33)$ & $357.10(24)$ & $356.98(20)$ & $356.9(5)$ & \\
\hline$\omega_{c}\left({ }^{\circ}\right)$ & 169.29(11) & $169.215(95)$ & 169.194(73) & $169.3(2)$ & \\
\hline$M_{b, \text { adjust }}\left({ }^{\circ}\right)$ & $4.0426(48)$ & 4.0441(50) & $4.0459(39)$ & - & \\
\hline$M_{c, \text { adjust }}\left({ }^{\circ}\right)$ & $-3.2629(63)$ & $-3.2654(53)$ & $-3.2648(46)$ & - & \\
\hline$M_{b}^{*}\left(^{\circ}\right)$ & $337.01(41)$ & $337.12(30)$ & $337.28(24)$ & $337.4(6)$ & \\
\hline$M_{c}^{*}\left(^{\circ}\right)$ & $313.489(97)$ & $313.553(87)$ & $313.575(67)$ & $313.5(1)$ & \\
\hline$\Omega_{b}\left({ }^{\circ}\right)$ & 0 (fixed) & 0 (fixed) & 0 (fixed) & 0 (fixed) & \\
\hline$\Omega_{c}\left({ }^{\circ}\right)$ & $-1.37(15)$ & $-1.244(88)$ & $-1.268(75)$ & 0 (fixed) & \\
\hline$R_{b} / R_{S}$ & $0.07644(60)$ & $0.07766(30)$ & $0.07759(27)$ & $0.0825(1)$ & \\
\hline$R_{c} / R_{S}$ & $0.07498(60)$ & $0.07601(32)$ & $0.07595(28)$ & $0.0796(2)$ & \\
\hline$R_{b}^{*}\left(R_{\oplus}\right)$ & $7.91(24)$ & $8.27(11)$ & $8.252(94)$ & 11.1(1) & $8.22_{-0.18}^{+0.26}$ \\
\hline$R_{c}^{*}\left(R_{\oplus}\right)$ & $7.76(23)$ & $8.10(10)$ & $8.077(92)$ & $10.7(1)$ & $8.05_{-0.18}^{+0.15}$ \\
\hline$\rho_{b}^{*}\left(\mathrm{~g} \mathrm{~cm}^{-3}\right)$ & $0.495(47)$ & $0.434(17)$ & $0.439(15)$ & $0.18(1)$ & \\
\hline$\rho_{c}^{*}\left(\mathrm{~g} \mathrm{~cm}^{-3}\right)$ & $0.362(34)$ & $0.319(12)$ & $0.322(11)$ & $0.14(1)$ & \\
\hline$\rho_{b}^{*}\left(\mathrm{~g} \mathrm{~cm}^{-3}\right)\left(\sigma_{m_{S}}\right.$ prop. $)$ & $0.495(60)$ & $0.434(24)$ & $0.439(23)$ & $0.18(1)$ & \\
\hline$\rho_{c}^{*}\left(\mathrm{~g} \mathrm{~cm}^{-3}\right)\left(\sigma_{m_{S}}\right.$ prop. $)$ & $0.362(44)$ & $0.319(18)$ & $0.322(17)$ & $0.14(1)$ & \\
\hline
\end{tabular}

Notes. Given are the median and standard deviation values from the MCMC posterior distributions. For the stellar radius and density, the bibliographic values are taken from Havel et al. (2011). The quadratic limb darkening coefficients are taken from Mullally et al. (2015). The osculating orbital elements are given at a reference time, BJD $=2454933.0 .{ }^{(*)}$ Derived, not fitted parameters.

area. Additionally, the difference between the best model of each of the data sets II and III can be seen in the bottom panels of the plots (henceforth "residual plots"). Comparing the yellow and black areas shows a slight narrowing of the model variation for data set III, which is reflected by the slightly smaller error bars in Table 3. In the case of Kepler-9b, the transit models slightly shift towards earlier transits when all ground-based data are included. This can be seen by comparing the yellow and black areas, but it is more obvious in the residual plots. A larger change between modelling the different data sets appears for Kepler-9c. The residuals of the best model for this transit show an asymmetric difference between the modelling of data sets II and III. This means an adjustment not only in the transit time, but also in the transit shape.

The obtained detrended ground-based transit light curves are shown in Fig. A.1, together with the best photodynamical model in grey, and the variation of 500 randomly chosen good fitting models in black. The data corresponding to Kepler-9b are plotted in red, and the ones of Kepler-9c are plotted in blue. Each observation has its own sub-figure, where the date and the used telescope are indicated. The transits that were observed from different sites simultaneously are artificially shifted to allow for a visual inspection. Raw photometry is available for download. 

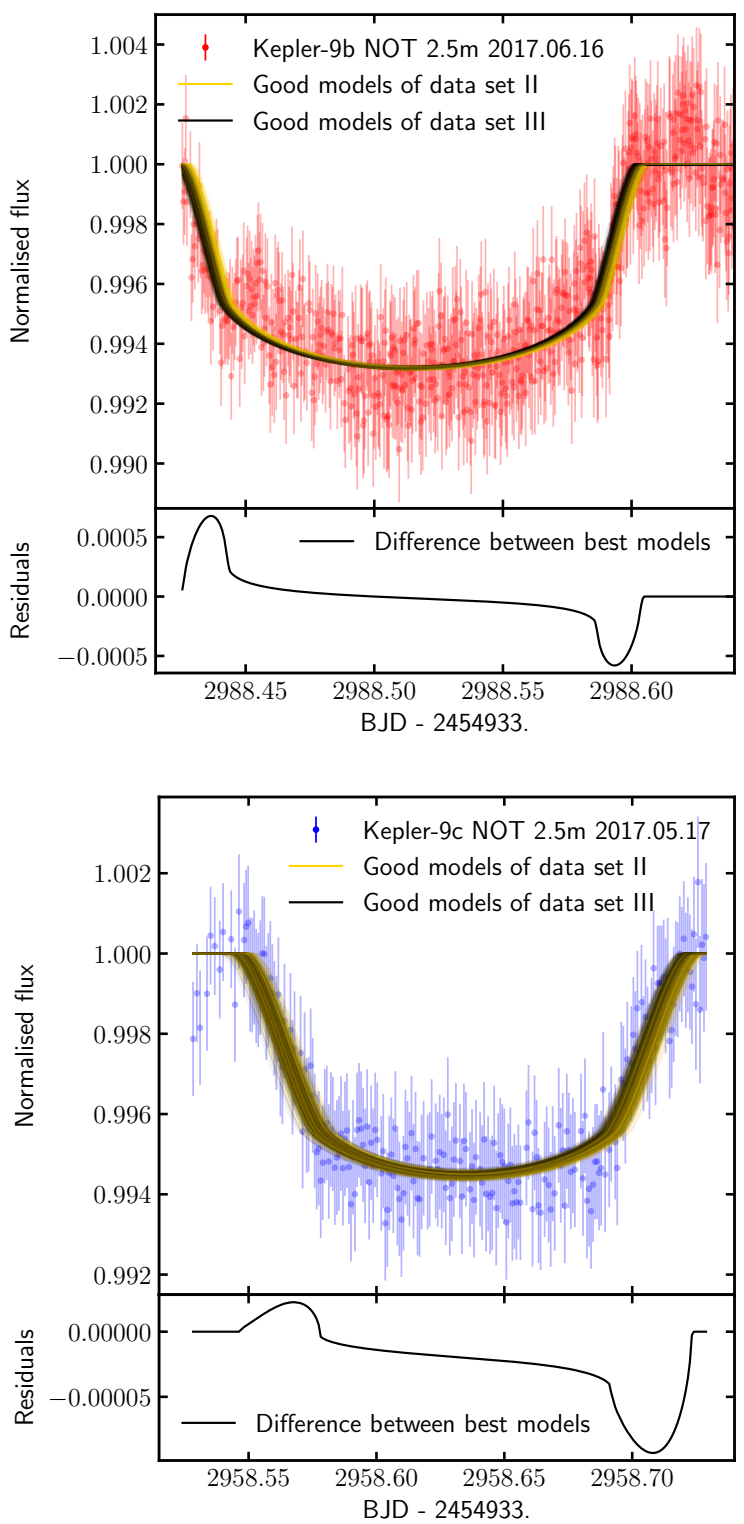

Fig. 2. Examples of newly obtained transit light curves for Kepler-9b in red (top), observed on June 16, 2017, and Kepler-9c in blue (bottom), observed on May 17, 2017. Both transits were observed using the NOT $2.5 \mathrm{~m}$ telescope. Overplotted is the variation of 500 randomly chosen good models by modelling data set II (yellow) and data set III (black). The residuals plot shows the difference between the best models of these two data sets.

Another derivable parameter of our photodynamical model is the transit times. Figure 3 shows the O-C diagram of the transit times measured by individually fitting the Kepler data, as well as the newly obtained ground-based data, in comparison to the results of modelling data set III. Also included is the mid-transit time of Kepler-9b obtained by Wang et al. (2018b), about $2 \sigma$ off from our model and our new data. Unfortunately, the photometric data are not published so we could not include them in our photodynamic analysis. The top part of Fig. 3 shows the $\mathrm{O}-\mathrm{C}$ diagram with the transit times from Kepler data in orange for Kepler-9b and in light blue for Kepler-9c. The O-C data from the new KOINet observations are shown in red for Kepler-9b and blue for Kepler-9c. The mid-time derived by Wang et al. (2018b) is shown in pink. The transit times from the best photodynamical model of data set III minus the linear trend are presented as grey lines. The middle part of this figure shows the residuals for Kepler-9b with the same colour identification, and the residuals of Kepler-9c are shown respectively in the bottom part. In both residual plots, the $99.74 \%$ confidence interval of 1000 randomly chosen good models of the different data sets in comparison to the best model of data set III are plotted as grey areas. The light grey area belongs to the modelling of data set I, middle grey to data set II, and dark grey to data set III. The differences in the amplitude of the variations of the models compared to the best model are discussed in the following section. In Table A.1, we provide transit-time predictions from modelling data set III for the next $10 \mathrm{yr}$.

\section{Discussion}

The results of the photodynamical modelling of Kepler-9b/c require some interpretation. In this section, we first discuss the dynamical stability of the derived system model, and subsequently we discuss the transit timing variations along with their prediction for future observations. We also specifically discuss the transit shape variations and the consequential prediction of disappearing transits for Kepler-9c. Moreover, we address the stellar activity and, connected to this, we investigate the stellar mass, radius, and age. The age is explored from stellar evolution models, as well as gyrochronologically. As the photodynamical modelling yields precise densities, our derived values are also the subject of discussion. Furthermore, the available radial velocity measurements of this system have not been mentioned in this paper; the reasons behind this choice are addressed below as well. The last point of this section deals with the innermost confirmed planet of the system, which is not included in the analysis. Finally, we discuss the possibility of detecting other planets in the system by means of the observed TTVs of Kepler-9b/c.

\subsection{Dynamical stability}

A dynamical analysis leads naturally to the question of the longterm stability of the derived planetary system, as an unstable result should not be considered as a viable model, contradicting the long lifetime of the system. To test the stability of our results for the Kepler-9 system, our best photodynamical solution was extended in time up to 1 Gyr. For this purpose, we used the second-order mixed-variable symplectic algorithm implemented in the Mercury6 package by Chambers (1999). This is the same integrator used in our photodynamical model. The postNewtonian correction (Kidder 1995) has also been included for this application. The time step size we used was 0.9 days, which is slightly smaller than a twentieth of the period of the innermost planet considered in our dynamical analysis, Kepler-9b. This step size is a good compromise between reasonable computation time and small integration errors. We find that, over the integration time, the modelled planetary system remains stable. Given the architecture of the system, this was expected, and we can assume that the very similar good results from MCMC modelling should remain stable as well.

\subsection{Transit timings}

After the Kepler observations, as time progresses, good MCMC models differ from the best data set III model at varying amplitude (see for instance the time range around 2014-2015, and 


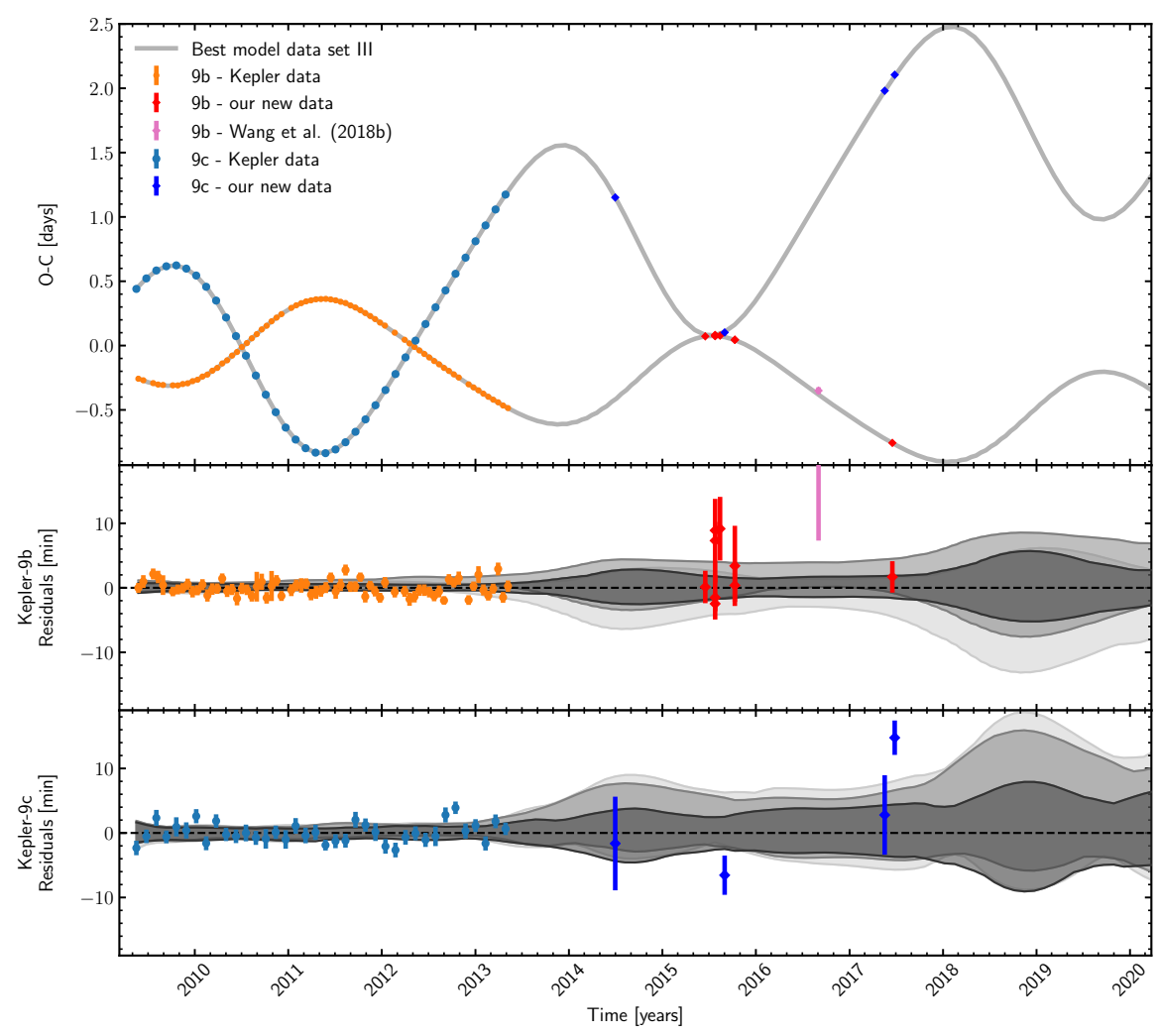

Fig. 3. O-C diagram of transit times from photodynamical modelling of data set III and the predictions until 2020 (top). Calculated (C) are transit times from a linear ephemeris modelled at the transit times found by Dreizler \& Ofir (2014). The residual plots (middle: Kepler-9b; bottom: Kepler-9c) show the $99.74 \%$ confidence interval of 1000 randomly chosen good models in comparison with the best model of data set III. From light to dark grey: modelling of data set I, II, and III. The Kepler transit times are derived by single transit modelling. The new transit time data points originate from the first analysis described in Sect. 2.3. around 2018-2020; Fig. 3). These variations are illustrated by the grey areas in different shades for the modelling of the different data sets, from light to dark grey corresponding to data sets I, II, and III. At the specific times previously mentioned, the variations increase for both planets. This behaviour appears when the $\mathrm{O}-\mathrm{C}$ has a positive slope for Kepler-9b, and a negative slope for Kepler-9c. At these places, the gradient of the TTVs is larger in comparison to the parts where Kepler-9b shows decreasing TTVs and when Kepler-9c shows increasing TTVs. A larger gradient leads to a larger uncertainty in the predictions. Despite the lower precision in comparison to the space-based Kepler data, the new ground-based KOINet observations help to set tighter constraints on the modulation of the timings. Unfortunately, apart from one observation, we missed the chance to observe transits in the phase of higher variation amplitude in 2014. The next period of higher-amplitude variation starts in 2018; a few more observations during 2018, especially of Kepler-9c transits, will help to further tighten constraints on this modulation.

\subsection{The disappearance of Kepler-9c transits}

One of the advantages of our photodynamical modelling is the physical consistency in modelling variations in the transit shape due to variations in the transit parameters. These variations can be explained by the dynamical interaction of all objects in the system. Figure 4 shows these variations in the transit shape. Plotted are the transit light curve data per planet shifted by their individual transit time. For a better visualisation of this effect, we plotted only Kepler quarters 1-17 of the long-cadence data. The higher scatter of short-cadence data would lead to a larger range in flux. In turn, the variation of the model would appear diminished due to the larger data range. The model variation is shown in black, which is the best model for each of the transits modelled in data set III, shifted to a common time of transit. For Kepler-9c especially, a clear variation in the transit shape is visible, both in transit depth and transit duration.

The variation in transit shape is not only most visible from long-cadence data, but also most significant. The same TTV model with an averaged transit-shape model gives a $8 \%$ worse reduced $\chi^{2}$ on data set I. On data sets II and III, the difference in the reduced $\chi^{2}$ is only of the order of $0.5 \%$. Nevertheless, photodynamical modelling has the advantage of consistently modelling the TTVs with the transit-shape-determining parameters, that is, mainly the inclination.

The observed variation in the transit shape of Kepler-9c leads us to examine the evolution of transit parameters over time. Figure 5 shows the variations of the semi-major axis, the eccentricity, and the inclination with the predictions for the next $50 \mathrm{yr}$. The predictions for the inclination of Kepler-9c show a continuous decrease, so that both the derived impact parameter $b$ and the transit duration indicate the disappearance of the transits around the year 2052. This behaviour is shown in Fig. 5 as well. A long-term inspection reveals the variations in inclination to be a periodic effect, meaning that the transits will return around 2230 again (see Fig. A.2). Through the decreasing inclination, within the next $35 \mathrm{yr}$ we will have the opportunity to map the high latitudes and hence measure the limb of Kepler-9 with frequent transit observations of planet $c$. In these higher latitudes, the transit spends more time at the limb than in the case of a passage of the mid-point of the star. This fact could help us to obtain more information on the atmospheric structure at the limb. 

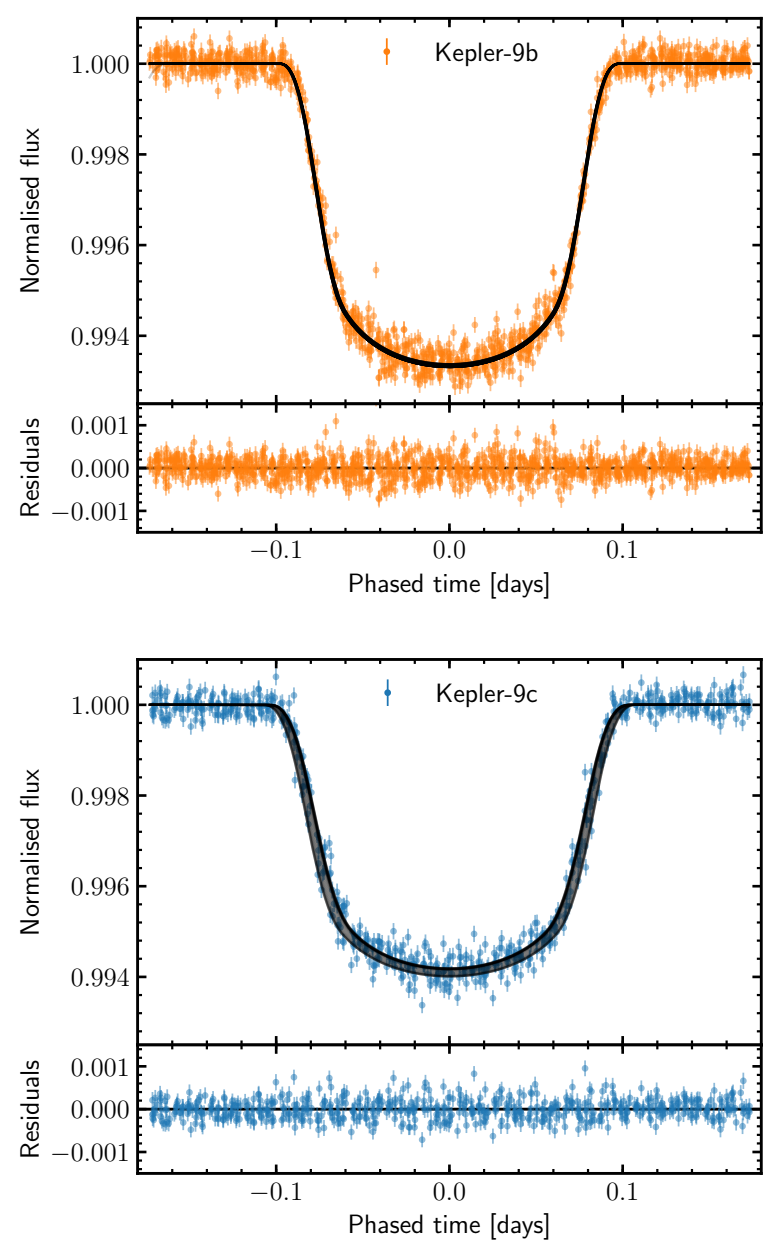

Fig. 4. All long-cadence Kepler (quarter 1-17) data per planet, aligned by the transit time, in orange for Kepler-9 $\mathrm{b}$ and in light blue for Kepler-9c. In grey is the best photodynamical model of the full data set, but calculated for only these Kepler long-cadence data and aligned respectively. The bottom of each figure shows the residuals.

On the other hand, for planet $b$, an increasing inclination in the next $100 \mathrm{yr}$ is predicted (see Fig. A.2). Wang et al. (2018a) measured a stellar spin alignment with the planetary orbital plane on a high probability. As this result might be affected by stellar spot crossings, more Rossiter-McLaughlin measurements are necessary for confirmation (Oshagh et al. 2016). Nevertheless, assuming spin alignment, Kepler-9b will scan the latitudes from its current location (above $30^{\circ}$ ), down to around $10^{\circ}$. With Kepler-9 being a solar analogue, a spot appearance similar to the sun between $0^{\circ}$ and $30^{\circ}$ is a reasonable assumption. Under those circumstances, we have a high probability of being able to measure spot crossings by Kepler-9b in precise transit observations in the future. Such detections would lead to a starspot distribution measurement like in the work of Morris et al. (2017) in which the system analysed, HAT-P-11, is known to be highly misaligned. Therefore, an even more similar analysis is possible if the spin alignment of Kepler-9 is not confirmed, in which case there could possibly be spot contamination in the existing transit observations. Spot crossings are not resolvably measured in these observations, meaning that a higher accuracy would be necessary for this analysis if they already occur.
The existing coverage of latitudes by transit observations is illustrated in Fig. A.3 under the assumption of a stellar spin alignment with the orbital plane. The red and blue lines refer to Kepler-9b and Kepler-9c transits, respectively. The track is extracted from the best photodynamical model of data set III. The uncertainties in these tracks are retrievable from the impact parameter shown in the fourth row of Fig. 5. The yellow circular disk illustrates the star and the orange area shows the possible star spot occurrence ranges assuming a similar behaviour to the sun.

The precise Kepler data allow us to model the quadratic limb darkening of the star. As a result, from modelling data set III, the derived limb darkening coefficients are $c_{1}=0.35 \pm 0.05$ and $c_{2}=0.27 \pm 0.07$. Figure A. 8 shows that these two coefficients are highly anti-correlated. This result is consistent with Müller et al. (2013), who investigated the quadratic limb darkening of Kepler targets. Additionally, the values suit the literature values given in the NASA Exoplanet Archive (Mullally et al. 2015). The results from modelling data set I demonstrate that using only long-cadence Kepler data is not sufficient to model the quadratic limb darkening of Kepler-9. Nonetheless, the derived values of $c_{1}=0.28 \pm 0.05$ and $c_{2}=0.41 \pm 0.09$ fit the anti-correlation derived by modelling data set III. This anticorrelation is illustrated in the parameter correlation plot in Fig. A.8. Consequently, the discrepant values lead to different results for the stellar radius and the planet-star radius ratios.

In order to check for model-dependent influences on the resulting evolution of the system parameters, we investigated the differences between Newtonian gravity and the inclusion of a post-Newtonian correction. An analysis was done for the influence on resulting photodynamical models for Kepler-9b and $\mathrm{c}$. Including the post-Newtonian correction decreased the parameter uncertainties in the second significant figure and the reduced $\chi^{2}$ in the fifth. The differences are too small to discriminate between the models. The future predictions for changes in inclination and for transit times behave very similarly.

\subsection{Stellar radius, mass, and age}

Applying our photodynamical analysis to data set III, we determined a stellar density of $\rho_{S}=1.603 \pm 0.061 \mathrm{~g} \mathrm{~cm}^{-3}$. As described in Sect. 3, we modelled the stellar radius instead of the density. However, the transit measurements constrain the stellar density (Agol \& Fabrycky 2017). With a fixed stellar mass, the density can be determined straightforwardly. Our modelled density is almost 50\% higher than the prior estimate $\left(\rho_{S}=(0.79 \pm 0.19) \rho_{\odot} \hat{=} 1.12 \pm 0.27 \mathrm{~g} \mathrm{~cm}^{-3}\right)$ by Havel et al. (2011). The authors derived this value from the TTV analysis of the first three quarters of Kepler observations by Holman et al. (2010). With this density, the stellar mass, radius, and age were determined by stellar evolution models. Our considerably higher derived density motivated a new, similar study of Kepler-9.

We used the stellar density and the known stellar parameters of an effective temperature $T_{\text {eff }}=5777 \pm 61 \mathrm{~K}$, surface gravity $\log g=4.49 \pm 0.09$ and metallicity $\mathrm{Fe} / \mathrm{H}=0.12 \pm 0.04$ (which classifies Kepler-9 as a solar analogue; see Holman et al. 2010; Havel et al. 2011) to determine the age, mass, and radius of the star by stellar evolution models. The results are presented in the Appendix A (similar to Figs. 6 and 7 derived below) as a massage diagram in Fig. A.4 and as a radius-age diagram in Fig. A.5. We extracted the corresponding values from the interpolated MESA (Paxton et al. 2011, 2013, 2015) evolutionary tracks by MIST (Dotter 2016; Choi et al. 2016). We derive a stellar mass 

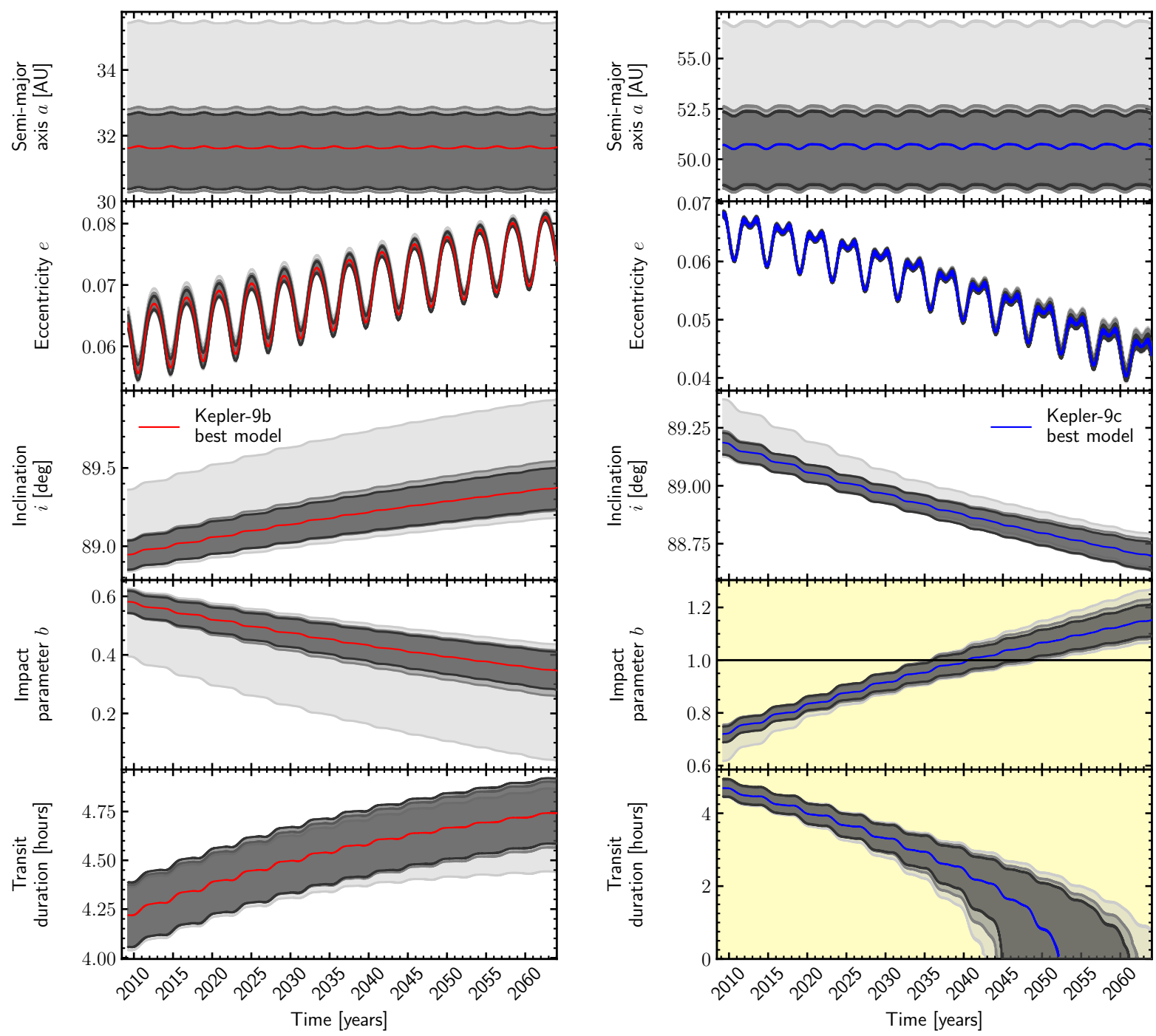

Fig. 5. Top: extrapolation of the planets semi-major axis until 2065 for the best model for Kepler-9b (red, left) and Kepler-9c (blue, right). Grey areas show the $99.74 \%$ confidence interval of 1000 randomly chosen good models for the different data sets. From light to dark grey: modelling of data sets I, II, and III. Second from top: extrapolation of the planets eccentricity. Third from top: extrapolation of the planets inclination. Fourth from top: extrapolation of the calculated impact parameter. Bottom: extrapolation of the calculated transit duration. The background of the impact parameter and the transit duration is coloured to highlight the place where the prediction of disappearing transits comes from.

of $m_{S}=1.06_{-0.05}^{+0.06} M_{\odot}$, a radius of $R_{S}=0.977_{-0.024}^{+0.031} R_{\odot}$ and an age of $\tau_{\text {Evol }}=0.95_{-0.92}^{+1.88}$ Gyr.

Recent HIRES observations by Petigura et al. (2017) of more than $1000 \mathrm{KOIs}$ led to the correction of the Kepler-9 stellar parameters to $T_{\text {eff }}=5787 \pm 60 \mathrm{~K}, \log g=4.473 \pm 0.1$, and $\mathrm{Fe} / \mathrm{H}=0.082 \pm 0.04$. Although very similar, the lower metallicity leads to slightly different results. With these new values, we determined the stellar mass to $m_{S}=1.04_{-0.05}^{+0.07} M_{\odot}$, the radius to $R_{S}=0.971_{-0.021}^{+0.030} R_{\odot}$ and the stellar age to $\tau_{\text {Evol }}=1.49_{-1.47}^{+2.15} \mathrm{Gyr}$. The corresponding diagrams can be found for mass versus age in Fig. 6 and for radius versus age in Fig. 7. We note that mass and radius for both parameter sets are in agreement within $1 \sigma$. The derived age of $1.5 \mathrm{Gyr}$, however, is in better agreement with the gyrochronological age derived below. With these new values for the stellar mass and radius, we corrected the modelled planetary masses, semi-major axes, and radii, which can be found in the sixth column of Table 3 .

More recently, the second Gaia data release (Gaia DR2) was carried out (Gaia Collaboration 2016, 2018). The effective temperature of $T_{\text {eff }}=5750_{-130}^{+250} \mathrm{~K}$ derived using DR2 data fits the HIRES value within the $1 \sigma$ range, as does the stellar radius with $R_{S}=0.977_{-0.080}^{+0.045}$. These values have comparatively higher uncertainties, however. The distance of Kepler-9 is determined to $p=1.563 \pm 0.017$ mas by Gaia DR2.

To test the results of the stellar evolution model analysis, we determined the gyrochronological age of Kepler-9. For this, we computed a periodogram of Kepler-9's full long-cadence photometry (Lomb 1976; Scargle 1982; Zechmeister \& Kürster 2009). The highest power peak corresponds to $16.83 \pm 0.08$ days. The period and error correspond to the mean and standard deviation obtained fitting a Gaussian to the highest periodogram peak. 


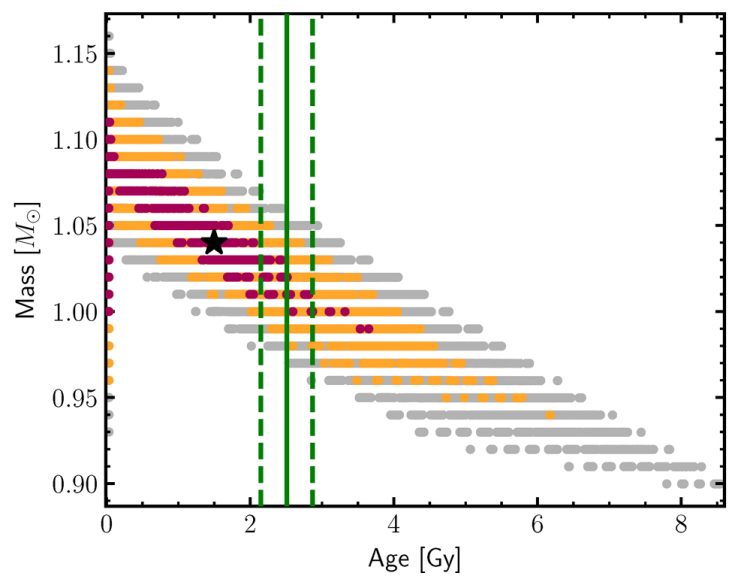

Fig. 6. Mass-age diagram of Kepler-9 from MESA stellar evolution models (MIST). The black star and the red, orange, and grey dots correspond to the best-matching value and the 1,2 , and $3 \sigma$ areas, respectively derived from results on the density of the data set III photodynamical modelling and from new literature values of the effective temperature, the surface gravity, and the metallicity by Petigura et al. (2017). The gyrochronological age is indicated by the green solid line and its $1-\sigma$ range with the green dashed lines.

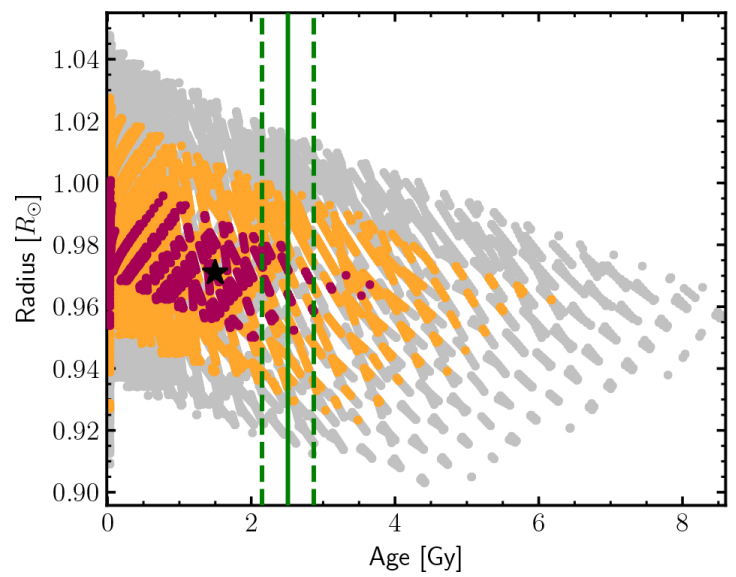

Fig. 7. Radius-age diagram of Kepler-9 from MESA stellar evolution models (MIST). The black star and the red, orange, and grey dots correspond to the best matching value and the 1,2 , and $3 \sigma$ areas, respectively, derived from results on the density of the data set III photodynamical modeling and from new literature values of the effective temperature, the surface gravity, and the metallicity by Petigura et al. (2017). The gyrochronological age is indicated by the green solid line and its $1-\sigma$ range with the green dashed lines.

On Kepler-9, typical photometric variability due to spot rotation has an amplitude of 5 ppt, well above the photometric noise.

To determine Kepler-9's age we made use of Barnes (2007, 2009)'s gyrochronological estimate:

$\log \left(\tau_{\text {Gyro }}\right)=\frac{1}{n}[\log P-\log a-b \times \log (\mathrm{B}-\mathrm{V}-c)]$,

for $a=0.770 \pm 0.014, b=0.553 \pm 0.052, c=0.472 \pm 0.027$ and $n=0.519 \pm 0.007$. Assuming $B-V=0.642$, and following Barnes (2009) error estimates, the derived gyrochronological age for Kepler-9 is $2.51 \pm 0.36$ Gyr. This age is indicated in the massage and radius-age diagrams (Figs. 6, 7, A.4, and A.5) by green

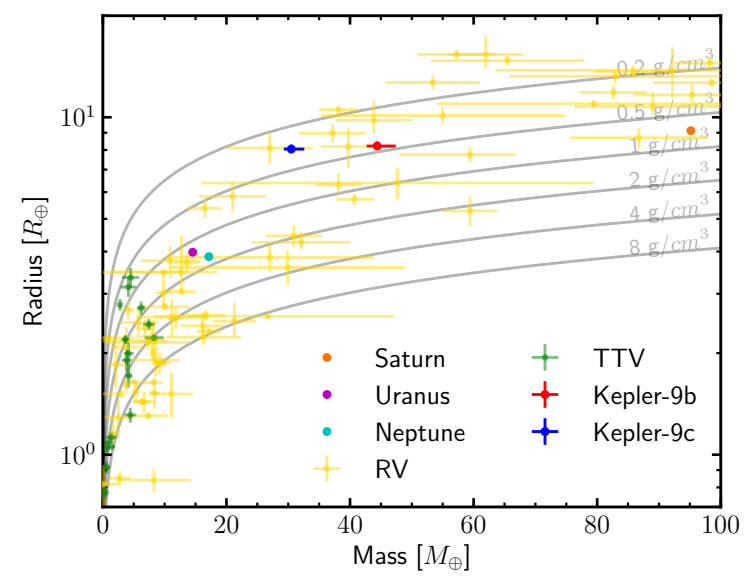

Fig. 8. Mass-radius diagram for known planets with masses up to $100 M_{\oplus}$. In yellow are the planets with mass measurements obtained by radial velocities and in green the planets with mass measurements obtained from TTVs. The data are given by the The Extrasolar Planets Encyclopaedia. Our results are shown in red (Kepler-9b) and in blue (Kepler-9c). For comparison also the values of Saturn, Uranus, and Neptune are shown, the Neptune-like planet pair of our solar system.

lines, solid for the the median value and dashed for the $1-\sigma$ range. The gyrochronological age is slightly higher than the age indicated by stellar evolution models, but the values agree within the $1-\sigma$ range.

\subsection{Stellar and planetary densities}

In addition to the stellar density, the photodynamical analysis provides strong constraints on the planetary densities. As a result of the analysis performed on data set III, we obtain densities of $\rho_{b}=0.439 \pm 0.023 \mathrm{~g} \mathrm{~cm}^{-3}$ for Kepler-9b and $\rho_{c}=0.322 \pm 0.017 \mathrm{~g} \mathrm{~cm}^{-3}$ for Kepler-9c. In Fig. 8 our results are compared to literature values from The Extrasolar Planets Encyclopaedia ${ }^{4}$ for planets with similar properties. Colourcoded are the mass measurements obtained from radial velocities in yellow, and from TTVs in green. In this regime, that is the regime of Neptune-like planets, the density measurements of Kepler-9b/c are, to date, the most precise ones outside the solar system.

To rule out biased results for stellar radius and planet-star radii ratios caused by the photometric variability of Kepler- 9 , we checked for variability in the residuals of the transit light curves. For consistency, we chose the high-precision, wellsampled Kepler short-cadence data for this analysis. The scatter of the residuals inside the transit is slightly larger than outside the transit. For the best model of data set III, the standard deviation inside the transit is $\operatorname{std}_{\text {inside }}=0.001049$, while outside it is $\operatorname{std}_{\text {outside }}=0.001027$, meaning a $2 \%$ difference between inside and outside the transit. We did not find any periodicity inside the transit residuals, potentially due to star spots. Equivalently, the transit time residuals do not show a periodic variability. Nevertheless, the higher scatter inside transit possibly results from unresolved stellar spot crossings. The planet-star radii ratio determination is affected within its uncertainties. With the absence of measurable star spots and the small differences in standard deviation between inside and outside the transit, a systematic error in

$\overline{4 \text { http://www.exoplanet.eu/ }}$ 


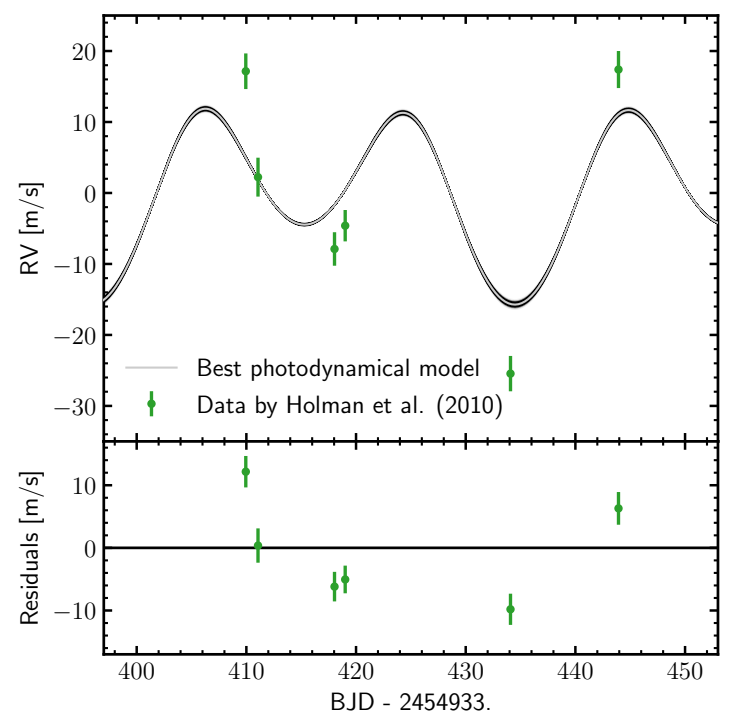

Fig. 9. Results from photodynamical modelling of data set III on the radial velocity measurements by Holman et al. (2010).

the radius determination seems to be negligible. The planetary densities are therefore also well determined.

Figure 8 shows the similarity in radius of the Kepler-9b/c planets to Saturn. The masses are less than half the value of Saturn, resulting in smaller densities. Their low density implies Kepler-9b/c should be classified as hydrogen-helium gas giants. The formation of the planets happened most likely in the outer region of the system. Through converging migration, the planets could be brought in the near 2:1 mean motion resonance in close proximity to the host star (e.g. shown by Henrard 1982; Borderies \& Goldreich 1984; Lemaitre 1984). It has been shown that such formation scenarios can result in stable resonant orbits with the outer planet having only about half the mass of the inner one (Deck \& Batygin 2015).

\subsection{Radial velocity measurements}

In our analysis, we did not consider the radial velocity (RV) measurements by Holman et al. (2010) for Kepler-9. The reasons are the small number of measurements, the short time span of the observations, as well as the large discrepancy between a dynamical model to the TTVs and the RV data. Nevertheless, from our photodynamical model we calculated an RV model. Simulated RV models from the results of modelling the full transit dataset are shown together with the data in Fig. 9. The best model has a $\chi^{2}=56.94$ for the six RV measurements. As pointed out by Dreizler \& Ofir (2014), we also see a similar discrepancy between the dynamical model and these measurements. Additional evidence in favour of the TTV model comes from the short-timescale chopping variations seen in planet $9 \mathrm{~b}$ due to $9 \mathrm{c}$ : the amplitude of chopping indicates a smaller mass for planet $9 \mathrm{c}$ (Deck \& Agol 2015), which, of course, is included in the full photodynamical constraint.

The most evident reason for this discrepancy is the activity of Kepler-9. A jitter factor would be necessary to include these data in the analysis. A detailed analysis of the activity of the star and the integration of the RV measurements is however, beyond the scope of this paper.
In addition, the recently obtained RV measurements listed in the HARPS-N archive ${ }^{5}$, but marked as proprietary, could help to better-constrain the RV behaviour of Kepler-9. Figure A.6 shows a prediction of the Kepler- 9 radial velocity based on our model constraints for the approximate time span of the new HARPS-N observations.

\subsection{An additional planet?}

To complete our analysis, we also tested the influence of the third known planet, Kepler-9d (confirmed by Torres et al. 2011) with a period of $P_{d}=1.592960$ (2) d, on the dynamics of the system. We agree with Dreizler \& Ofir (2014) that it does not interact measurably with the two modelled planets, in the plausible mass regime $\left(m_{d}=1-7 M_{\oplus}\right.$, Holman et al. 2010). For a mass of $m_{d}=7 M_{\oplus}$, the reduced $\chi^{2}$ does not improve and also the variations of the residuals of the transit times are of the same order. The amplitude in radial velocity measurements is of the order of $1 \mathrm{~m} \mathrm{~s}^{-1}$, far below the precision of the previous observations and currently unfeasible for a star as faint as Kepler-9.

Adding another outer planet in a Laplace-resonance $(4: 2: 1)$ to explain the deviations in the radial velocity measurements would require a rather high mass for the additional planet. Such a planet would have far too large an influence on the system's dynamics and is ruled out by the photodynamical analysis. The fact that only six RV measurements are published makes it impossible to set constraints on further possible planets. Additional planets could exist outside the Laplace-resonance, thereby explaining the discrepancies between transit and radial velocity measurements, yet not substantially influencing the short-term dynamics. In addition we find no periodicity in the transit timing residuals, whereas evidence of periodicy here would have been a sign of an outer planet.

\section{Conclusions}

With this work, we substantiate the importance of the KOINet. With its anticorrelated, large-amplitude TTVs, the Kepler-9b/c system was chosen as a benchmark system for the photodynamical modelling. Although the dynamical cycle was almost covered by Kepler observations, the 13 new transit observations led to better constraints on the composition of the system. Concurrently, we have confirmed the capability of KOINet to complete a transit observation with a long duration by using several telescopes around the globe. This is complemented by the results of the photodynamical modelling. The application to Kepler-9 revealed that the transits of the outer planet will disappear in about $30 \mathrm{yr}$. Furthermore, this dynamical analysis of the combined photometric data, consisting of Kepler long- and short-cadence data in addition to ground-based follow-up observations led to the most precise planetary density measurements of planets in the Neptune-mass regime so far.

From the decreasing inclination of Kepler-9c and increasing inclination of Kepler-9b we have the opportunity to map the different latitudes of the star. Therefore, measurements of the limb and the star spots of Kepler-9 could be made possible by precise, frequent transit observations within the next $35 \mathrm{yr}$ for the limb and $100 \mathrm{yr}$ for the star spots. Interspersed with frequent groundbased follow-up, transit measurements from space that provide a high photometric precision would complement the stellar analysis. The promising predictions of this work make Kepler-9 an

5 http://archives.ia2 .inaf.it/tng/faces/search. xhtml? dswid=9814 
interesting target for space missions like TESS (Ricker et al 2015), PLATO 2.0 (Rauer et al. 2014), or CHEOPS (Broeg et al. 2013), though it is a relatively faint object, with a Kepler magnitude of $K_{p}=13.803$.

Acknowledgements. We acknowledge funding from the German Research Foundation (DFG) through grant DR 281/30-1. This work made use of PyAstronomy. $\mathrm{CvE}$ acknowledges funding for the Stellar Astrophysics Centre, provided by The Danish National Research Foundation (Grant DNRF106). Based on observation made with the Nordic Optical Telescope, operated by the Nordic Optical Telescope Scientific Association at the Observatorio del Roque de los Muchachos, La Palma, Spain, of the Instituto de Astrofísica de Canarias. We acknowledge support from the Research Council of Norway's grant 188910 to finance service observing at the NOT. SW acknowledges support for International Team 265 ("Magnetic Activity of M-type Dwarf Stars and the Influence on Habitable Extrasolar Planets) funded by the International Space Science Institute (ISSI) in Bern, Switzerland. EA acknowledges support from United States National Science Foundation grant 1615315. EH and IR acknowledge support by the Spanish Ministry of Economy and Competitiveness (MINECO) and the Fondo Europeo de Desarrollo Regional (FEDER) through grant ESP2016-80435-C2-1-R, as well as the support of the Generalitat de Catalunya/CERCA programme. The Joan Oro Telescope (TJO) of the Montsec Astronomical Observatory (OAdM) is owned by the Generalitat de Catalunya and operated by the Institute for Space Studies of Catalonia (IEEC). Based on observations collected at the Centro Astronómico Hispano Alemán (CAHA) at Calar Alto, operated jointly by the Max-Planck Institut für Astronomie and the Instituto de Astrofísica de Andalucía (CSIC) Based on observations obtained with the Apache Point Observatory $3.5 \mathrm{~m}$ telescope, which is owned and operated by the Astrophysical Research Consortium. The Liverpool Telescope is operated on the island of La Palma by Liverpoo John Moores University in the Spanish Observatorio del Roque de los Muchachos of the Instituto de Astrofisica de Canarias with financial support from the UK Science and Technology Facilities Council.

\section{References}

Agol, E., \& Fabrycky, D. 2017, in Handbook of Exoplanets, eds. H. J. Deeg \& J. A. Belmonte (Springer Living Reference Work), 7

Agol, E., Steffen, J., Sari, R., \& Clarkson, W. 2005, MNRAS, 359, 567

Barnes, S. A. 2007, ApJ, 669, 1167

Barnes, S. A. 2009, IAU Symp., 258, 345

Borderies, N., \& Goldreich, P. 1984, Celest. Mech., 32, 127

Borsato, L., Marzari, F., Nascimbeni, V., et al. 2014, A\&A, 571, A38

Borucki, W. J., Koch, D., Basri, G., et al. 2010, Science, 327, 977

Broeg, C., Fortier, A., Ehrenreich, D., et al. 2013, Eur. Phys. J. Web Conf., 47, 03005

Carter, J. A., \& Winn, J. N. 2009, ApJ, 704, 51

Chambers, J. E. 1999, MNRAS, 304, 793

Choi, J., Dotter, A., Conroy, C., et al. 2016, ApJ, 823, 102

Deck, K. M., \& Agol, E. 2015, ApJ, 802, 116

Deck, K. M., \& Batygin, K. 2015, ApJ, 810, 119

Deck, K. M., Agol, E., Holman, M. J., \& Nesvorný, D. 2014, ApJ, 787,
Dotter, A. 2016, ApJS, 222, 8

Dreizler, S., \& Ofir, A. 2014, ArXiv e-prints [arXiv: 1403.1372]

Eastman, J., Siverd, R., \& Gaudi, B. S. 2010, PASP, 122, 935

Foreman-Mackey, D., Hogg, D. W., Lang, D., \& Goodman, J. 2013, PASP, 125 306

Gaia Collaboration (Prusti, T., et al.) 2016, A\&A, 595, A

Gaia Collaboration (Brown, A. G. A., et al.) 2018, A\&A, 616, A1

Gillon, M., Jehin, E., Lederer, S. M., et al. 2016, Nature, 533, 221

Goodman, J., \& Weare, J. 2010, Commun. Appl. Math. Comput. Sci., 5, 65

Hadden, S., \& Lithwick, Y. 2017, AJ, 154, 5

Havel, M., Guillot, T., Valencia, D., \& Crida, A. 2011, A\&A, 531, A3

Henrard, J. 1982, Celest. Mech., 27, 3

Holman, M. J., \& Murray, N. W. 2005, Science, 307, 1288

Holman, M. J., Fabrycky, D. C., Ragozzine, D., et al. 2010, Science, 330,

Jontof-Hutter, D., Ford, E. B., Rowe, J. F., et al. 2016, ApJ, 820, 39

Kidder, L. E. 1995, Phys. Rev. D, 52, 821

Kipping, D. M. 2010, MNRAS, 408, 1758

Kjeldsen, H., \& Frandsen, S. 1992, PASP, 104, 413

Latham, D. W., Rowe, J. F., Quinn, S. N., et al. 2011, ApJ, 732, L24

Lemaitre, A. 1984, Celest. Mech., 34, 329

Lissauer, J. J., Fabrycky, D. C., Ford, E. B., et al. 2011a, Nature, 470, 53

Lissauer, J. J., Ragozzine, D., Fabrycky, D. C., et al. 2011b, ApJS, 197, 8

Lomb, N. R. 1976, Ap\&SS, 39, 447

Mandel, K., \& Agol, E. 2002, ApJ, 580, L17

Mazeh, T., Nachmani, G., Holczer, T., et al. 2013, ApJS, 208, 16

Mills, S. M., \& Mazeh, T. 2017, ApJ, 839, L8

Morris, B. M., Hebb, L., Davenport, J. R. A., Rohn, G., \& Hawley, S. L. 2017, ApJ, 846, 99

Mullally, F., Coughlin, J. L., Thompson, S. E., et al. 2015, ApJS, 217, 31

Müller, H. M., Huber, K. F., Czesla, S., Wolter, U., \& Schmitt, J. H. M. M. 2013 A\&A, 560, A112

Oshagh, M., Dreizler, S., Santos, N. C., Figueira, P., \& Reiners, A. 2016, A\&A, 593, A25

Paxton, B., Bildsten, L., Dotter, A., et al. 2011, ApJS, 192, 3

Paxton, B., Cantiello, M., Arras, P., et al. 2013, ApJS, 208, 4

Paxton, B., Marchant, P., Schwab, J., et al. 2015, ApJS, 220, 15

Petigura, E. A., Howard, A. W., Marcy, G. W., et al. 2017, AJ, 154

Rauer, H., Catala, C., Aerts, C., et al. 2014, Exp. Astron., 38, 249

Ricker, G. R., Winn, J. N., Vanderspek, R., et al. 2015, J. Astron. Telesc. Instruments Syst., 1, 014003

Scargle, J. D. 1982, ApJ, 263, 835

Shallue, C. J., \& Vanderburg, A. 2018, AJ, 155, 94

Southworth, J., Hinse, T. C., Jørgensen, U. G., et al. 2009, MNRAS, 396 1023

Steele, I. A., Smith, R. J., Rees, P. C., et al. 2004, Proc. SPIE, 5489, 679

Torres, G., Fressin, F., Batalha, N. M., et al. 2011, ApJ, 727, 24

von Essen, C., Schröter, S., Agol, E., \& Schmitt, J. H. M. M. 2013, A\&A, 555 A92

von Essen, C., Ofir, A., Dreizler, S., et al. 2018, A\&A, 615, A79

Wang, S., Addison, B., Fischer, D. A., et al. 2018a, AJ, 155, 70

Wang, S., Wu, D.-H., Addison, B. C., et al. 2018b, AJ, 155, 73

Zechmeister, M., \& Kürster, M. 2009, A\&A, 496, 577 
J. Freudenthal et al.: Kepler Object of Interest Network. II.

Appendix A: Additional plots and a table of the transit time predictions
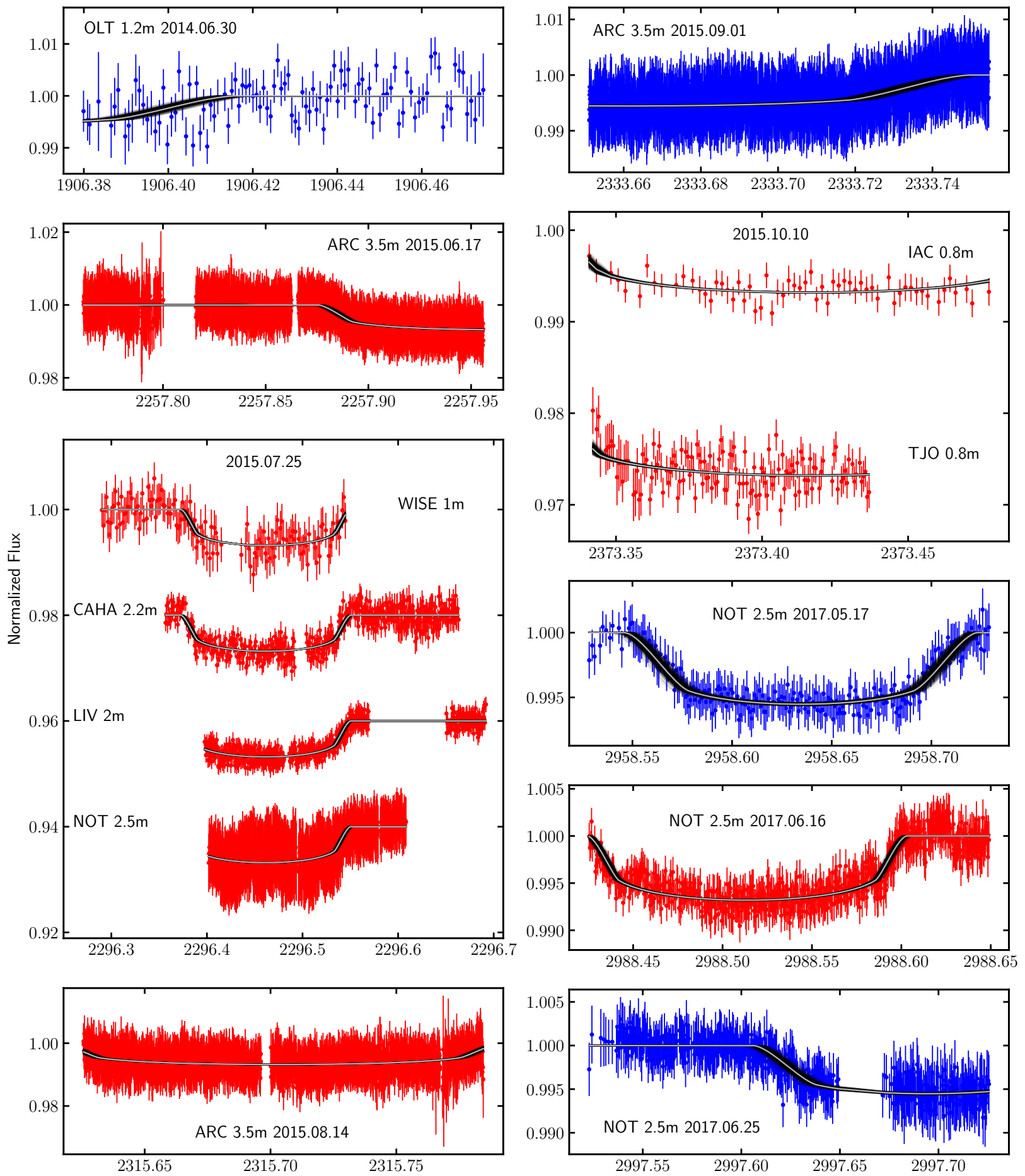

BJD - 2454933.0

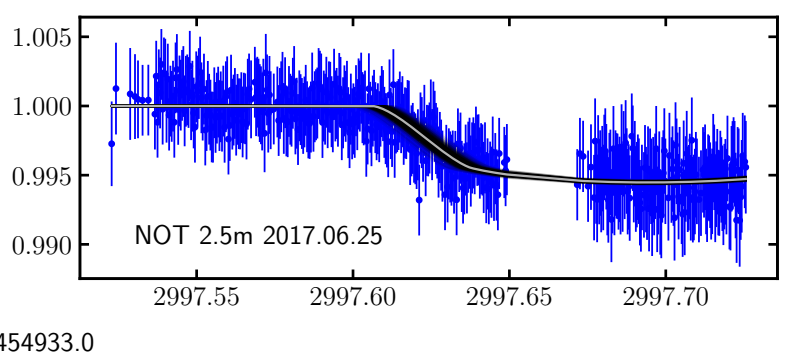

Fig. A.1. Our observed transits with the best model of data set III in grey and its variations by 500 randomly chosen good models in black. Transit data in red belong to Kepler-9b and blue transit data correspond to Kepler-9c. The transits from dates with more than one observation are artificially shifted for better visualisation and the telescope used is indicated. 

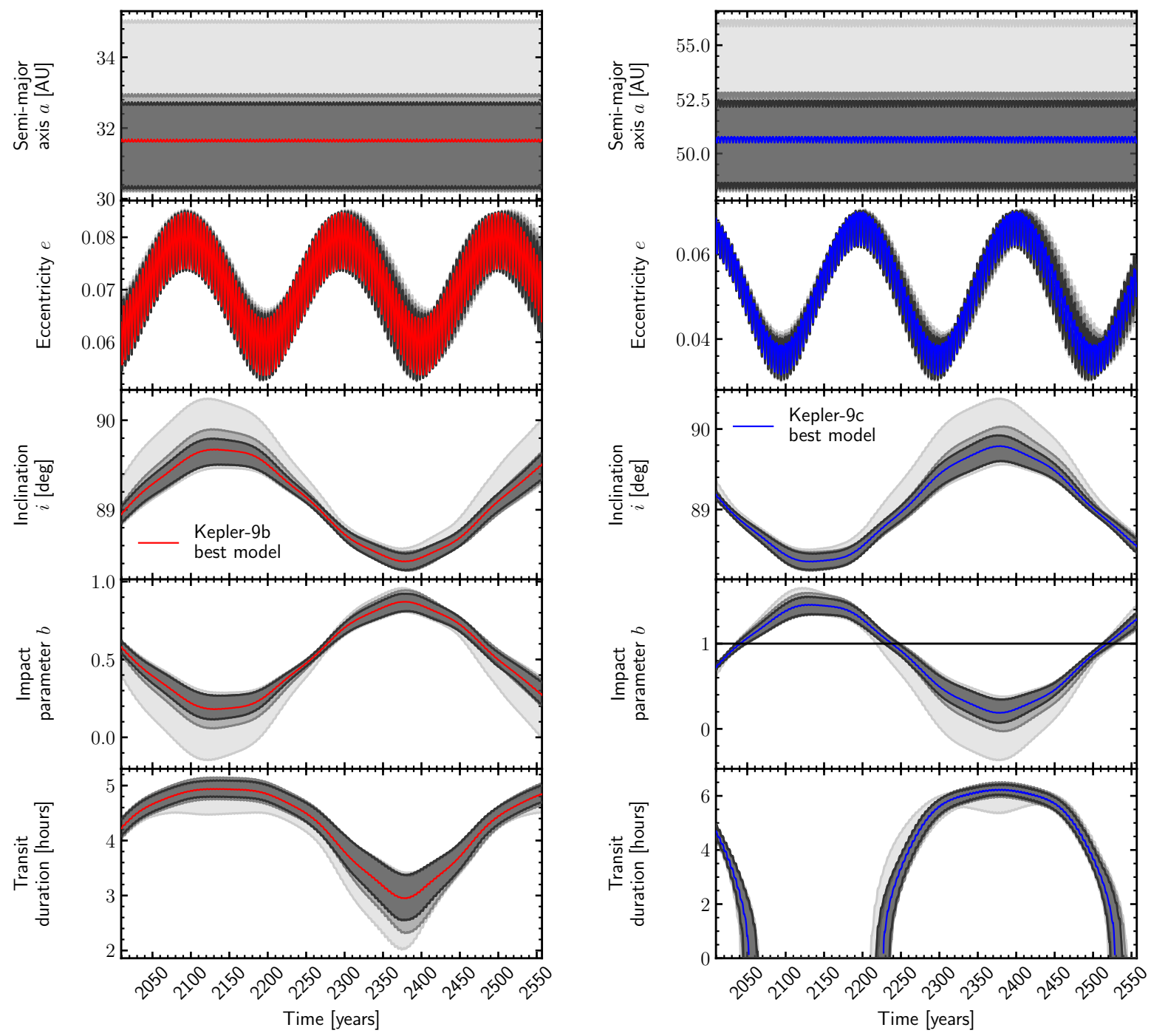

Fig. A.2. Top: extrapolation of the planets semi-major axis until 2550 for the best model in red (Kepler-9b) and blue (Kepler-9c) and in grey areas the $99.74 \%$ confidence interval of 1000 randomly chosen good models for the different data sets. From light to dark grey: modelling of data sets I, II, and III. Second from top: extrapolation of the planets' eccentricity. Third from top: extrapolation of the planets inclination. Fourth from top: extrapolation of the calculated impact parameter. Bottom: extrapolation of the calculated transit duration. 
J. Freudenthal et al.: Kepler Object of Interest Network. II.

Table A.1. Ephemerides E and transit time predictions in BJD-2400000.0 from modelling data set III for the next $10 \mathrm{yr}$.

\begin{tabular}{|c|c|c|c|c|c|c|c|}
\hline $\mathrm{E}$ & BJD & $\mathrm{E}$ & BJD & $\mathrm{E}$ & BJD & $\mathrm{E}$ & BJD \\
\hline \multicolumn{8}{|c|}{ Kepler-9b } \\
\hline 165 & $58133.0822(7)$ & 222 & $59230.2853(6)$ & 279 & $60327.6320(13)$ & 336 & $61423.9735(30)$ \\
\hline 166 & $58152.3330(7)$ & 223 & $59249.5049(6)$ & 280 & $60346.8684(13)$ & 337 & $61443.2492(31)$ \\
\hline 167 & $58171.5797(8)$ & 224 & $59268.7261(6)$ & 281 & $60366.0990(12)$ & 338 & $61462.5362(31)$ \\
\hline 168 & $58190.8374(9)$ & 225 & $59287.9463(7)$ & 282 & $60385.3303(12)$ & 339 & $61481.8137(31)$ \\
\hline 169 & $58210.0897(9)$ & 226 & $59307.1682(7)$ & 283 & $60404.5561(11)$ & 340 & $61501.1012(31)$ \\
\hline 170 & $58229.3543(10)$ & 227 & $59326.3890(7)$ & 284 & $60423.7831(11)$ & 341 & $61520.3792(31)$ \\
\hline 171 & $58248.6125(10)$ & 228 & $59345.6117(7)$ & 285 & $60443.0054(11)$ & 342 & $61539.6657(31)$ \\
\hline 172 & $58267.8836(11)$ & 229 & 59364.8334(7) & 286 & 60462.2291(11) & 343 & $61558.9429(31)$ \\
\hline 173 & $58287.1474(12)$ & 230 & $59384.0569(7)$ & 287 & $60481.4489(10)$ & 344 & $61578.2269(31)$ \\
\hline 174 & $58306.4240(12)$ & 231 & 59403.2799(7) & 288 & $60500.6702(10)$ & 345 & $61597.5020(30)$ \\
\hline 175 & $58325.6928(13)$ & 232 & $59422.5044(7)$ & 289 & $60519.8885(10)$ & 346 & $61616.7820(29)$ \\
\hline 176 & $58344.9740(13)$ & 233 & $59441.7290(7)$ & 290 & $60539.1082(10)$ & 347 & $61636.0537(29)$ \\
\hline 177 & $58364.2468(13)$ & 234 & $59460.9550(7)$ & 291 & $60558.3259(10)$ & 348 & $61655.3282(28)$ \\
\hline 178 & $58383.5311(14)$ & 235 & $59480.1818(7)$ & 292 & $60577.5447(10)$ & 349 & $61674.5955(27)$ \\
\hline 179 & $58402.8069(14)$ & 236 & $59499.4096(8)$ & 293 & $60596.7623(10)$ & 350 & $61693.8635(26)$ \\
\hline 180 & $58422.0930(14)$ & 237 & $59518.6395(8)$ & 294 & $60615.9806(10)$ & 351 & $61713.1253(25)$ \\
\hline 181 & $58441.3706(14)$ & 238 & $59537.8696(8)$ & 295 & $60635.1985(10)$ & 352 & $61732.3857(24)$ \\
\hline 182 & $58460.6569(14)$ & 239 & 59557.1034(9) & 296 & $60654.4168(10)$ & 353 & $61751.6414(23)$ \\
\hline 183 & $58479.9349(14)$ & 240 & 59576.3366(9) & 297 & 60673.6353(10) & 354 & $61770.8939(22)$ \\
\hline 184 & $58499.2199(14)$ & 241 & $59595.5752(10)$ & 298 & $60692.8538(10)$ & 355 & $61790.1431(21)$ \\
\hline 185 & $58518.4971(14)$ & 242 & $59614.8123(10)$ & 299 & $60712.0730(10)$ & 356 & $61809.3878(20)$ \\
\hline 186 & $58537.7793(14)$ & 243 & $59634.0566(11)$ & 300 & $60731.2918(10)$ & 357 & $61828.6306(20)$ \\
\hline 187 & $58557.0542(14)$ & 244 & 59653.2983(11) & 301 & $60750.5118(10)$ & 358 & $61847.8682(19)$ \\
\hline 188 & $58576.3322(13)$ & 245 & $59672.5491(12)$ & 302 & $60769.7310(10)$ & 359 & $61867.1050(18)$ \\
\hline 189 & $58595.6037(13)$ & 246 & $59691.7960(13)$ & 303 & $60788.9518(10)$ & 360 & $61886.3363(17)$ \\
\hline 190 & $58614.8760(13)$ & 247 & $59711.0537(14)$ & 304 & $60808.1716(10)$ & 361 & $61905.5678(17)$ \\
\hline 191 & $58634.1429(12)$ & 248 & $59730.3063(15)$ & 305 & $60827.3932(10)$ & 362 & $61924.7941(16)$ \\
\hline 192 & $58653.4085(12)$ & 249 & $59749.5708(16)$ & 306 & 60846.6137(10) & 363 & $61944.0213(16)$ \\
\hline 193 & $58672.6698(11)$ & 250 & 59768.8292(17) & 307 & $60865.8362(10)$ & 364 & $61963.2438(15)$ \\
\hline 194 & $58691.9279(11)$ & 251 & $59788.1003(18)$ & 308 & $60885.0576(10)$ & 365 & $61982.4676(15)$ \\
\hline 195 & $58711.1829(10)$ & 252 & $59807.3642(18)$ & 309 & 60904.2811(10) & 366 & $62001.6874(15)$ \\
\hline 196 & $58730.4333(10)$ & 253 & $59826.6410(19)$ & 310 & $60923.5038(10)$ & 367 & $62020.9088(14)$ \\
\hline 197 & $58749.6817(10)$ & 254 & $59845.9098(20)$ & 311 & $60942.7283(10)$ & 368 & $62040.1269(14)$ \\
\hline 198 & $58768.9246(9)$ & 255 & $59865.1912(21)$ & 312 & $60961.9527(10)$ & 369 & $62059.3466(14)$ \\
\hline 199 & 58788.1666(9) & 256 & $59884.4640(21)$ & 313 & $60981.1787(11)$ & 370 & $62078.5639(14)$ \\
\hline 200 & $58807.4026(8)$ & 257 & $59903.7488(22)$ & 314 & $61000.4054(11)$ & 371 & $62097.7826(14)$ \\
\hline 201 & $58826.6387(8)$ & 258 & $59923.0246(22)$ & 315 & $61019.6333(11)$ & 372 & $62116.9998(14)$ \\
\hline 202 & $58845.8688(8)$ & 259 & $59942.3112(22)$ & 316 & $61038.8630(12)$ & 373 & $62136.2179(14)$ \\
\hline 203 & $58865.0999(7)$ & 260 & 59961.5887(22) & 317 & 61058.0934(12) & 374 & $62155.4354(13)$ \\
\hline 204 & $58884.3254(7)$ & 261 & $59980.8757(23)$ & 318 & $61077.3270(13)$ & 375 & $62174.6533(13)$ \\
\hline 205 & $58903.5523(7)$ & 262 & $60000.1537(22)$ & 319 & $61096.5605(13)$ & 376 & $62193.8714(13)$ \\
\hline 206 & $58922.7744(7)$ & 263 & $60019.4395(22)$ & 320 & $61115.7989(14)$ & 377 & $62213.0895(13)$ \\
\hline 207 & 58941.9981(7) & 264 & 60038.7167(22) & 321 & $61135.0363(15)$ & 378 & $62232.3083(13)$ \\
\hline 208 & $58961.2179(7)$ & 265 & $60057.9998(22)$ & 322 & $61154.2805(16)$ & 379 & $62251.5267(13)$ \\
\hline 209 & $58980.4393(7)$ & 266 & $60077.2749(21)$ & 323 & $61173.5225(17)$ & 380 & $62270.7463(13)$ \\
\hline 210 & $58999.6578(7)$ & 267 & $60096.5539(21)$ & 324 & $61192.7730(18)$ & 381 & $62289.9651(13)$ \\
\hline 211 & $59018.8777(7)$ & 268 & $60115.8255(21)$ & 325 & $61212.0202(19)$ & 382 & $62309.1856(13)$ \\
\hline 212 & $59038.0957(7)$ & 269 & $60135.0989(20)$ & 326 & $61231.2777(20)$ & 383 & $62328.4050(13)$ \\
\hline 213 & 59057.3147(7) & 270 & 60154.3660(19) & 327 & $61250.5305(21)$ & 384 & $62347.6263(13)$ \\
\hline 214 & $59076.5327(7)$ & 271 & 60173.6328(19) & 328 & $61269.7949(23)$ & 385 & $62366.8465(13)$ \\
\hline 215 & $59095.7514(7)$ & 272 & 60192.8943(18) & 329 & $61289.0534(24)$ & 386 & $62386.0687(13)$ \\
\hline 216 & 59114.9697(7) & 273 & 60212.1536(17) & 330 & $61308.3245(25)$ & 387 & $62405.2899(13)$ \\
\hline 217 & 59134.1883(7) & 274 & $60231.4089(17)$ & 331 & $61327.5884(26)$ & 388 & $62424.5131(14)$ \\
\hline 218 & $59153.4073(6)$ & 275 & $60250.6603(16)$ & 332 & $61346.8653(27)$ & 389 & $62443.7355(14)$ \\
\hline
\end{tabular}

Notes. The median and standard deviation solution of 1000 randomly chosen good models. Reference times for ephemeris $E=1$ : $T_{b}=54977.24962(54)$ and $T_{c}=54969.30566(78)$. 
Table A.1. continued.

\begin{tabular}{|c|c|c|c|c|c|c|c|}
\hline $\mathrm{E}$ & BJD & $\mathrm{E}$ & BJD & $\mathrm{E}$ & BJD & $\mathrm{E}$ & BJD \\
\hline \multicolumn{8}{|c|}{ Kepler-9c } \\
\hline 219 & $59172.6261(6)$ & 276 & $60269.9091(15)$ & 333 & $61366.1340(28)$ & 390 & $62462.9601(14)$ \\
\hline 220 & $59191.8458(6)$ & 277 & $60289.1529(14)$ & 334 & $61385.4156(29)$ & 391 & $62482.1842(14)$ \\
\hline 221 & $59211.0649(6)$ & 278 & $60308.3953(14)$ & 335 & $61404.6884(30)$ & 392 & $62501.4102(15)$ \\
\hline 82 & $58125.7907(10)$ & 111 & $59255.0764(17)$ & 140 & $60384.0857(19)$ & 169 & $61514.8518(47)$ \\
\hline 83 & $58164.7408(11)$ & 112 & $59294.1486(17)$ & 141 & $60423.1271(20)$ & 170 & $61553.6402(46)$ \\
\hline 84 & $58203.6605(12)$ & 113 & $59333.2194(17)$ & 142 & $60462.1834(21)$ & 171 & $61592.4390(44)$ \\
\hline 85 & $58242.5493(14)$ & 114 & $59372.2879(17)$ & 143 & $60501.2500(22)$ & 172 & $61631.2545(41)$ \\
\hline 86 & $58281.4089(16)$ & 115 & $59411.3529(17)$ & 144 & $60540.3229(23)$ & 173 & $61670.0929(38)$ \\
\hline 87 & $58320.2428(18)$ & 116 & $59450.4124(17)$ & 145 & $60579.3991(24)$ & 174 & $61708.9592(34)$ \\
\hline 88 & $58359.0557(20)$ & 117 & $59489.4635(17)$ & 146 & $60618.4764(25)$ & 175 & $61747.8572(30)$ \\
\hline 89 & $58397.8536(21)$ & 118 & $59528.5028(16)$ & 147 & $60657.5537(25)$ & 176 & $61786.7885(27)$ \\
\hline 90 & $58436.6427(22)$ & 119 & $59567.5263(16)$ & 148 & $60696.6301(25)$ & 177 & $61825.7525(25)$ \\
\hline 91 & $58475.4297(22)$ & 120 & $59606.5294(15)$ & 149 & $60735.7055(25)$ & 178 & $61864.7467(24)$ \\
\hline 92 & $58514.2212(21)$ & 121 & $59645.5080(16)$ & 150 & $60774.7795(25)$ & 179 & $61903.7666(25)$ \\
\hline 93 & $58553.0240(20)$ & 122 & $59684.4584(17)$ & 151 & $60813.8522(25)$ & 180 & $61942.8073(26)$ \\
\hline 94 & $58591.8444(18)$ & 123 & $59723.3785(20)$ & 152 & $60852.9232(24)$ & 181 & $61981.8635(28)$ \\
\hline 95 & $58630.6884(17)$ & 124 & $59762.2678(23)$ & 153 & $60891.9918(24)$ & 182 & $62020.9307(29)$ \\
\hline 96 & $58669.5608(15)$ & 125 & $59801.1279(26)$ & 154 & $60931.0567(24)$ & 183 & $62060.0046(30)$ \\
\hline 97 & $58708.4650(13)$ & 126 & $59839.9620(29)$ & 155 & $60970.1159(23)$ & 184 & $62099.0820(31)$ \\
\hline 98 & $58747.4020(12)$ & 127 & $59878.7749(32)$ & 156 & $61009.1667(23)$ & 185 & $62138.1607(32)$ \\
\hline 99 & $58786.3710(12)$ & 128 & $59917.5724(33)$ & 157 & $61048.2058(22)$ & 186 & $62177.2393(32)$ \\
\hline 100 & $58825.3689(12)$ & 129 & $59956.3608(34)$ & 158 & $61087.2292(21)$ & 187 & $62216.3170(32)$ \\
\hline 101 & $58864.3913(13)$ & 130 & $59995.1465(34)$ & 159 & $61126.2324(21)$ & 188 & $62255.3933(32)$ \\
\hline 102 & $58903.4329(14)$ & 131 & $60033.9364(33)$ & 160 & $61165.2112(22)$ & 189 & $62294.4682(32)$ \\
\hline 103 & $58942.4889(15)$ & 132 & $60072.7371(32)$ & 161 & $61204.1622(24)$ & 190 & $62333.5415(32)$ \\
\hline 104 & $58981.5547(16)$ & 133 & $60111.5551(29)$ & 162 & $61243.0830(28)$ & 191 & $62372.6128(32)$ \\
\hline 105 & $59020.6264(16)$ & 134 & $60150.3963(27)$ & 163 & $61281.9731(32)$ & 192 & $62411.6815(31)$ \\
\hline 106 & $59059.7012(17)$ & 135 & $60189.2658(24)$ & 164 & $61320.8338(37)$ & 193 & $62450.7463(30)$ \\
\hline 107 & $59098.7772(17)$ & 136 & $60228.1670(21)$ & 165 & $61359.6686(41)$ & 194 & $62489.8054(30)$ \\
\hline 108 & $59137.8532(17)$ & 137 & $60267.1014(19)$ & 166 & $61398.4818(44)$ & & \\
\hline 109 & $59176.9285(17)$ & 138 & $60306.0681(18)$ & 167 & $61437.2792(46)$ & & \\
\hline 110 & $59216.0030(17)$ & 139 & $60345.0643(18)$ & 168 & $61476.0670(47)$ & & \\
\hline
\end{tabular}


J. Freudenthal et al.: Kepler Object of Interest Network. II.

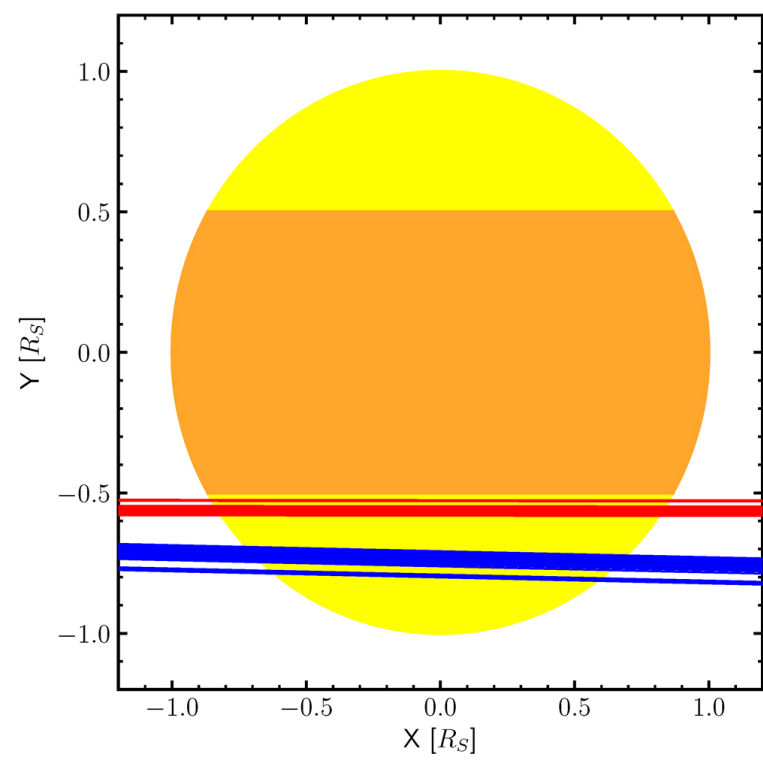

Fig. A.3. Latitude coverage of Kepler-9 (yellow circular disc) by al transit observations of data set III for Kepler-9b (red) and Kepler-9c (blue). Demonstrated is the best model of data set III. The order of the variations can be drawn from Figs. 5 or A.2, where the fourth row shows the modelled impact parameters. The orange area indicates the possible spot occurrence area between $0^{\circ}$ and $30^{\circ}$ up- and downwards.

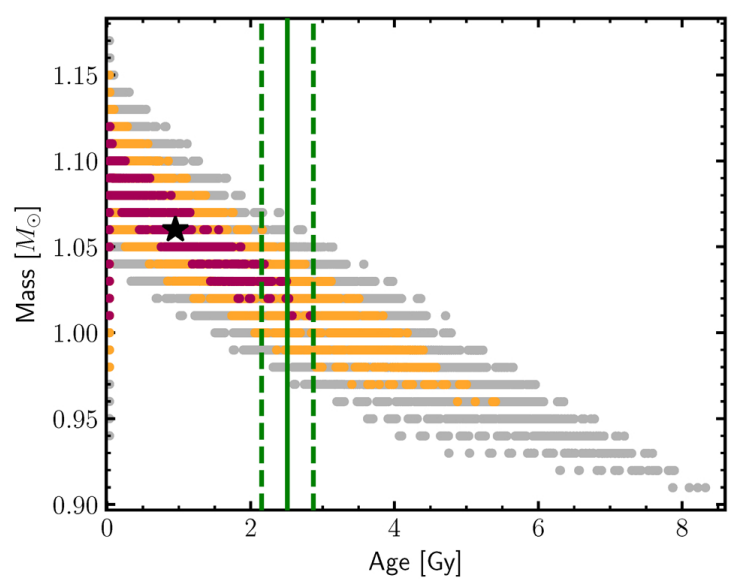

Fig. A.4. Mass-age diagram of Kepler-9 from MESA stellar evolution models (MIST). The black star and the red, orange, and grey dots correspond to the best matching value and the 1,2 , and $3 \sigma$ areas, respectively, derived from results on the density of the data set III photodynamical modelling and from literature values of the effective temperature, the surface gravity, and the metallicity by Holman et al. (2010). The gyrochronological age is indicated by the green solid line and its $1-\sigma$ range with the green dashed lines.

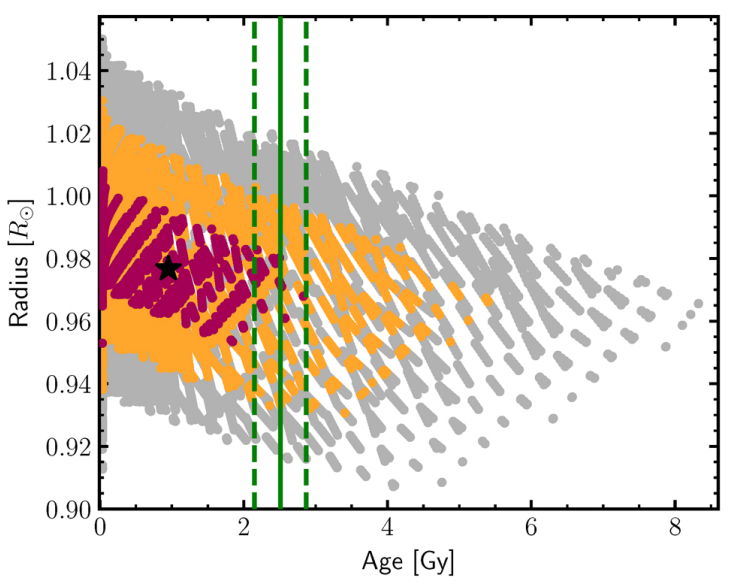

Fig. A.5. Radius-age diagram of Kepler-9 from MESA stellar evolution models (MIST). The black star and the red, orange, and grey dots correspond to the best matching value and the 1,2 , and $3 \sigma$ areas, respectively, derived from results on the density of the data set III photodynamical modelling and from literature values of the effective temperature, the surface gravity, and the metallicity by Holman et al. (2010). The gyrochronological age is indicated by the green solid line and its $1-\sigma$ range with the green dashed lines.

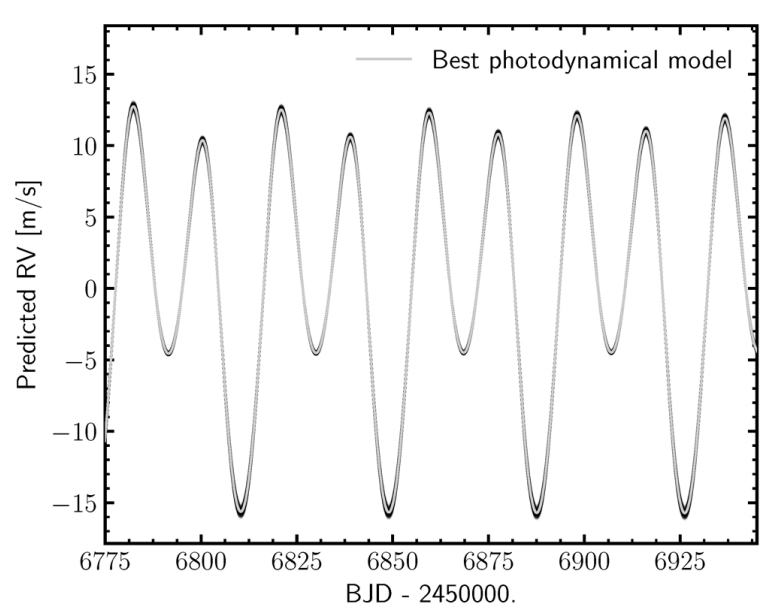

Fig. A.6. Predicted radial velocity measurements from the results of the photodynamical modelling of data set III for the approximate time span of the new observations listed in the HARPS-N archive 
A\&A 618, A41 (2018)

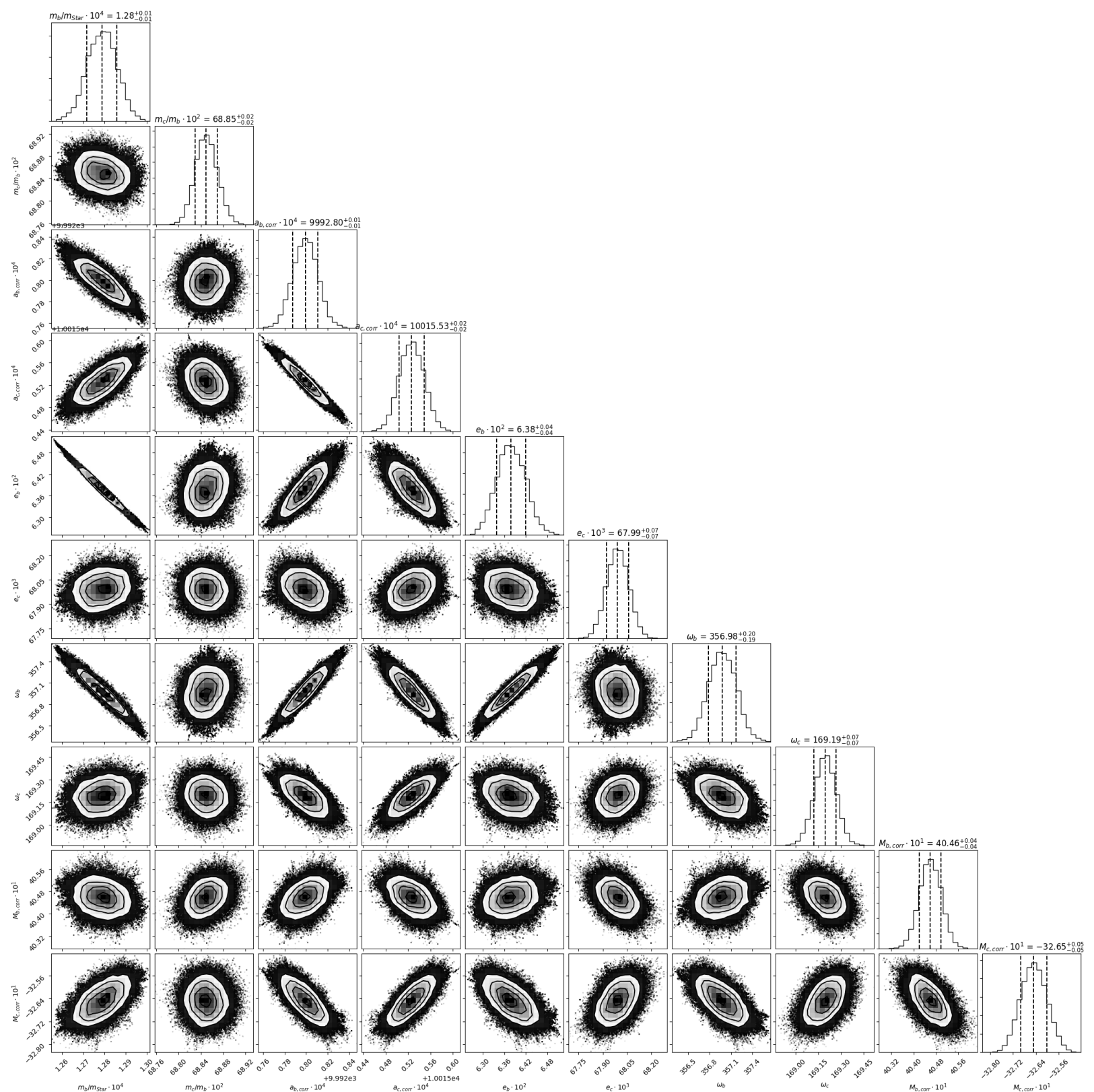

Fig. A.7. Correlation plot of masses, semi-major-axis, eccentricities, longitude of Periastron and mean anomaly from MCMC chains of modelling the full dataset. 
J. Freudenthal et al.: Kepler Object of Interest Network. II.

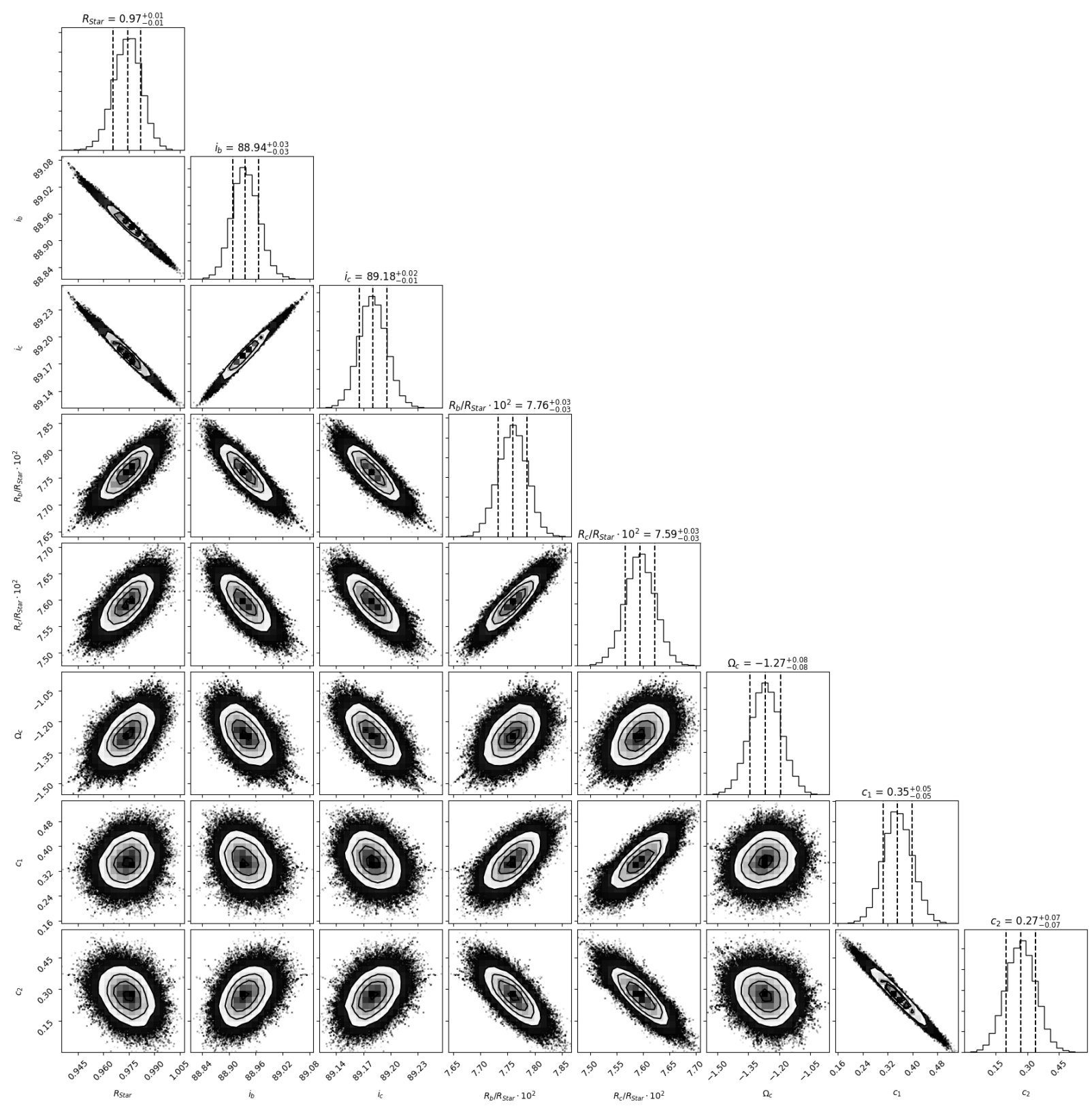

Fig. A.8. Correlation plot of stellar radius, inclination, planetary radii, argument of the ascending node of Kepler-9c and limb darkening coefficients from MCMC chains of modelling the full dataset. 


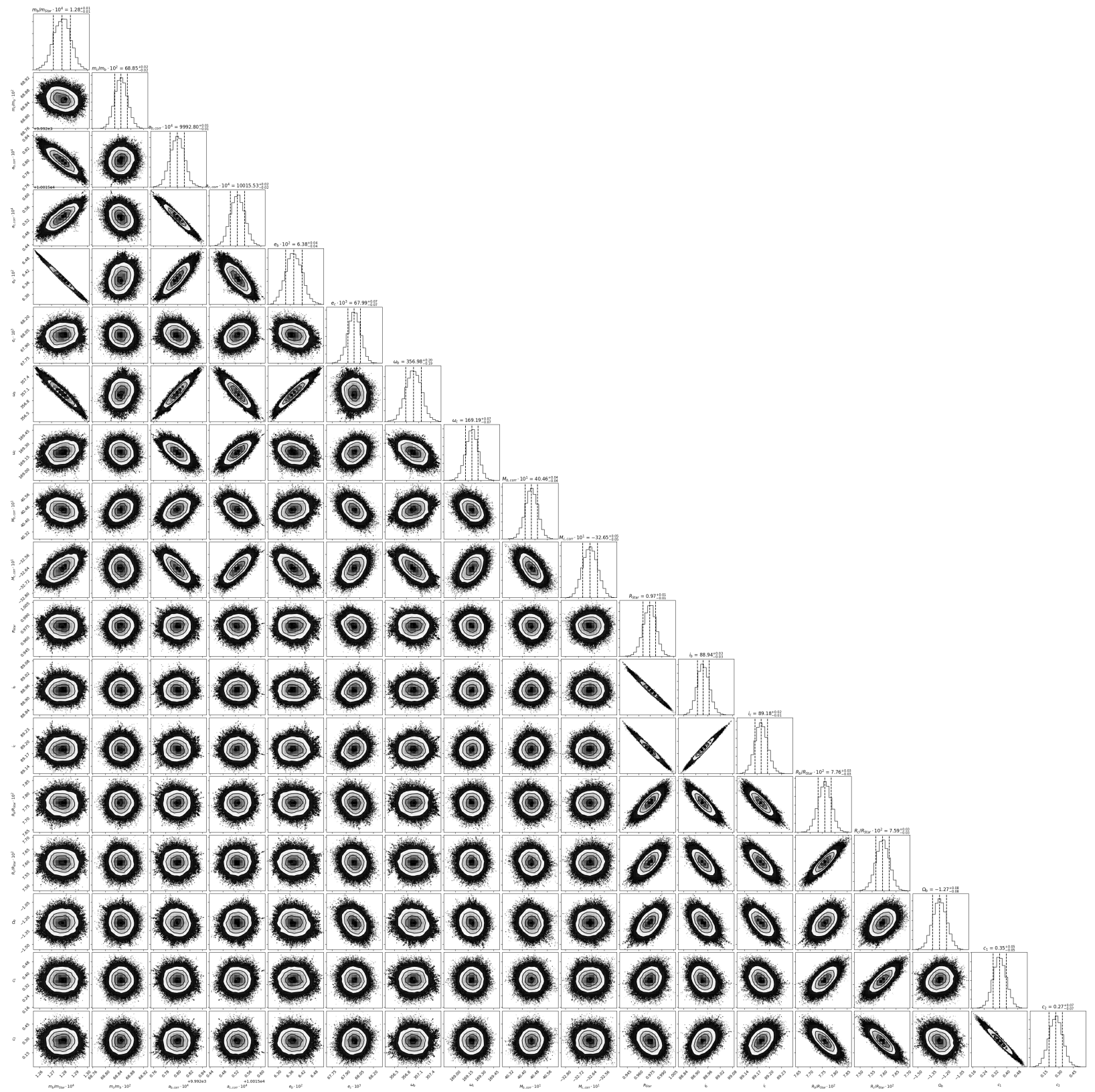

Fig. A.9. Full correlation plot of all fit parameters from modelling the full dataset. 


\section{Chapter 3}

\section{Kepler-82}

The section of this chapter has been published in Astrophysics \& Astronomy as the article 'Kepler Object of Interest Network III. Kepler-82f: A new non-transiting $21 M_{\oplus}$ planet from photodynamical modelling' (Freudenthal et al., 2019); Credit: J. Freudenthal et al., A\&A, 628, A108, 2019, reproduced with permission (C) ESO.

The publication was leaded in all stages by J. Freudenthal. The text was mainly written by J. Freudenthal. Section 1 was written in cooperation with C. von Essen. Text editing was done by all co-authors and a language editor assigned by A\&A. The KOINet data used in the paper were provided by all authors except J. Freudenthal and S. Dreizler. The KOINet data reduction and a first single transit analysis was conducted by C. von Essen in cooperation with J. Freudenthal. The photodynamical analysis and its interpretation was performed by J. Freudenthal under supervision of C. von Essen and S. Dreizler.

\subsection{Paper: Kepler Object of Interest Network III. Kepler-82f: A new non-transiting $21 M_{\oplus}$ planet from photodynamical modelling}




\title{
Kepler Object of Interest Network
}

\section{Kepler-82f: a new non-transiting $21 M_{\oplus}$ planet from photodynamical modelling ${ }^{\star}$}

\author{
J. Freudenthal ${ }^{1}$, C. von Essen ${ }^{2,1}$, A. Ofir ${ }^{3}$, S. Dreizler ${ }^{1}$, E. Agol ${ }^{4,5, \star \star}$, S. Wedemeyer ${ }^{6,7}$, B. M. Morris ${ }^{4}$,
} A. C. Becker ${ }^{4,8}$, H. J. Deeg ${ }^{9,10}$, S. Hoyer ${ }^{9}$, M. Mallonn ${ }^{11}$, K. Poppenhaeger ${ }^{11,12}$, E. Herrero ${ }^{13,14}$, I. Ribas ${ }^{13,14}$, P. Boumis ${ }^{15}$, and A. Liakos ${ }^{15}$

\author{
${ }^{1}$ Institut für Astrophysik, Georg-August-Universität Göttingen, Friedrich-Hund-Platz 1, 37077 Göttingen, Germany \\ e-mail: jfreude@astro.physik.uni-goettingen.de \\ 2 Stellar Astrophysics Centre, Aarhus University, Ny Munkegade 120, 8000 Aarhus, Denmark \\ 3 Department of Earth and Planetary Sciences, Weizmann Institute of Science, Rehovot 76100, Israel \\ 4 Astronomy Department, University of Washington, Seattle, WA 98195, USA \\ 5 Virtual Planetary Laboratory, University of Washington, Seattle, WA 98195, USA \\ ${ }^{6}$ Rosseland Centre for Solar Physics, University of Oslo, PO Box 1029 Blindern, 0315 Oslo, Norway \\ 7 Institute of Theoretical Astrophysics, University of Oslo, PO Box 1029 Blindern, 0315 Oslo, Norway \\ 8 Amazon Web Services, Seattle, WA 98121, USA \\ 9 Aix-Marseille University, CNRS, CNES, LAM, Marseille, France \\ ${ }^{10}$ Universidad de La Laguna, Departamento de Astrofísica, 38206 La Laguna, Tenerife, Spain \\ ${ }^{11}$ Leibniz-Institut für Astrophysik Potsdam, An der Sternwarte 16, 14482 Potsdam, Germany \\ 12 Astrophysics Research Centre, Queen's University Belfast, Belfast BT7 1NN, UK \\ ${ }^{13}$ Institut de Ciències de l'Espai (IEEC-CSIC), C/Can Magrans, s/n, Campus UAB, 08193 Bellaterra, Spain \\ ${ }^{14}$ Institut d'Estudis Espacials de Catalunya (IEEC), Gran Capità, 2-4, Edif. Nexus, 08034 Barcelona, Spain \\ 15 Institute for Astronomy, Astrophysics, Space Applications and Remote Sensing, National Observatory of Athens, \\ Metaxa \& Vas. Pavlou St., Penteli, Athens, Greece
}

Received 13 May 2019 / Accepted 20 June 2019

\section{ABSTRACT}

Context. The Kepler Object of Interest Network (KOINet) is a multi-site network of telescopes around the globe organised for followup observations of transiting planet candidate Kepler objects of interest with large transit timing variations (TTVs). The main goal of KOINet is the completion of their TTV curves as the Kepler telescope stopped observing the original Kepler field in 2013.

Aims. We ensure a comprehensive characterisation of the investigated systems by analysing Kepler data combined with new groundbased transit data using a photodynamical model. This method is applied to the Kepler- 82 system leading to its first dynamic analysis. Methods. In order to provide a coherent description of all observations simultaneously, we combine the numerical integration of the gravitational dynamics of a system over the time span of observations with a transit light curve model. To explore the model parameter space, this photodynamical model is coupled with a Markov chain Monte Carlo algorithm.

Results. The Kepler-82b/c system shows sinusoidal TTVs due to their near 2:1 resonance dynamical interaction. An additional chopping effect in the TTVs of Kepler-82c hints to a further planet near the 3:2 or 3:1 resonance. We photodynamically analysed Kepler long- and short-cadence data and three new transit observations obtained by KOINet between 2014 and 2018 . Our result reveals a non-transiting outer planet with a mass of $m_{\mathrm{f}}=20.9 \pm 1.0 M_{\oplus}$ near the 3:2 resonance to the outermost known planet, Kepler-82c. Furthermore, we determined the densities of planets $\mathrm{b}$ and $\mathrm{c}$ to the significantly more precise values $\rho_{\mathrm{b}}=0.98_{-0.14}^{+0.10} \mathrm{~g} \mathrm{~cm}^{-3}$ and $\rho_{\mathrm{c}}=0.494_{-0.077}^{+0.066} \mathrm{~g} \mathrm{~cm}^{-3}$.

Key words. planets and satellites: dynamical evolution and stability - planets and satellites: detection - methods: data analysis techniques: photometric - stars: individual: Kepler-82 - stars: fundamental parameters

\section{Introduction}

There is no doubt about the impact that the Kepler Space Telescope has had on the exoplanetary field. Among many other outstanding and benchmark contributions, such as the first possibly habitable planet with known radius (Borucki et al. 2012), and the first exoplanet ever found with two suns in its sky (Doyle et al. 2011), Kepler data have allowed us to characterise planetary

\footnotetext{
${ }^{\star}$ Ground-based photometry is only available at the CDS via anonymous ftp to cdsarc.u-strasbg.fr $(130.79 .128 .5)$ or via http: //cdsarc.u-strasbg.fr/viz-bin/qcat?]/A+A/628/A108 ${ }^{\star}$ Guggenheim Fellow.
}

masses via transit timing variations (TTVs, see e.g. Fabrycky et al. 2012; Mazeh et al. 2013; Steffen et al. 2013). Nonetheless, after four years of continuous monitoring of the same field of view, the nominal observations of Kepler came to an end. This left several Kepler objects of interest (KOIs) without a proper characterisation, even though they presented large amplitude TTVs in the Kepler data alone. To continue with the successful characterisation of planetary masses of KOIs via TTVs, we have organised the Kepler Object of Interest Network ${ }^{1}$ (KOINet). To date, results of our network comprise KOINet's first light

1 koinet.astro.physik. uni-goettingen.de 
(von Essen et al. 2018), and the in-depth photodynamical characterisation of Kepler-9b/c (Freudenthal et al. 2018). While in the former we demonstrated KOINet's strategy and functionality, along with initial results on four KOIs, in the latter we were able to determine values for the planetary densities that are the most precise measurements in the regime of Neptune-like exoplanets. Furthermore, we predicted that the transits of Kepler-9c would disappear in about $30 \mathrm{yr}$. These results arose from the combination of the Kepler long- and short-cadence data with KOINet follow-up transit observations, along with a comprehensive and coherent analysis carried out with our photodynamical modelling. Similar analyses have likewise revealed precise planetary densities for other systems, like Kepler-117 by Almenara et al. (2015), K2-19 by Barros et al. (2015), WASP-47 by Almenara et al. (2016), Kepler-138 by Almenara et al. (2018a), and Kepler-419 by Almenara et al. (2018b). In many of these cases the authors also demonstrated consistent planetary mass determinations from TTV and radial velocity (RV) measurements.

From amongst our KOINet targets we pinpointed Kepler-82 (KOI 0880) as an interesting system that deserves a detailed photodynamical analysis. The Kepler-82 system contains a total of four confirmed transiting planets. The two inner planets have periods of $P_{\mathrm{d}}=2.38 \mathrm{~d}$ and $P_{\mathrm{e}}=5.90 \mathrm{~d}$, which were confirmed by Rowe et al. (2014). The two outer planets have a period ratio close to $2: 1$ with $P_{\mathrm{b}}=26.44 \mathrm{~d}$ and $P_{\mathrm{c}}=51.54 \mathrm{~d}$. This commensurability of the periods results in strong TTVs (see Fig. 1), which led to the confirmation of the two outer planets a year before the inner planets (Xie 2013). The inner two planets are not much affected by this dynamical interaction and also show no measurable dynamical interaction with one another. Yet Kepler-82e shows TTVs with an amplitude of about $15 \mathrm{~min}$, where the uncertainties of the transit times are of the same order, and the variations are without significant periodicity (Holczer et al. 2016). Ofir et al. (2018) found TTVs in Kepler-82d with an amplitude of $10.3_{-1.4}^{+1.8} \mathrm{~min}$ and a frequency peak that just surpassed their significance criteria. The peak does not correspond to any expected dynamical frequency.

The first characterisation of the Kepler-82b/c TTVs was carried out by Xie (2013). The author found the TTVs to be sinusoidal as expected for near 2:1 mean-motion resonance (MMR) systems. In contrast with many other similar systems, the sinusoidal-shaped TTVs of both planets are not anti-correlated; instead the phase difference is close to zero. The author calculated the nominal masses assuming a two-planet system and found a relatively large mass ratio of $m_{\mathrm{b}} / m_{\mathrm{c}} \sim 10^{0.6} \sim 4$, which means a very large density ratio of $\rho_{\mathrm{b}} / \rho_{\mathrm{c}} \sim 4 \times(5.35 / 4)^{3} \sim$ 10 . Another nominal mass computation by Hadden \& Lithwick (2014) indicates a smaller mass $(\sim 3)$ and density ratio $(\sim 7)$.

A further characterisation was done by Ofir et al. (2018) by analysing periodograms of the TTVs of Kepler-82b/c. They found the most significant peak in the periodogram of Kepler-82b fits the 2:1 MMR super frequency. However, the highest amplitude peak of Kepler-82c is notably offset from the highest peak of Kepler- $82 b$ and the 2:1 MMR super frequency. Additionally, they found one other significant peak for Kepler-82b and three in Kepler-82c.

The following work includes the first dynamical analysis of the Kepler-82b/c system. We applied a photodynamical model to Kepler data and ground-based follow-up observations from KOINet. With this we were able to constrain the planetary masses more precisely, and by including another non-transiting planet, most of the frequency peaks, can be explained. Furthermore, we were able to determine the stellar mass, radius and age from our results by combining the modelled stellar densities with spectroscopic values and comparing these values with stellar evolution models.

The paper is structured as follows. The data acquisition and treatment within the KOINet is described in Sect. 2. We present our own implementation of a photodynamical model in Sect. 3. The detection of a third dynamically important non-transiting planet in the TTVs of Kepler-82c is described in detail in Sect. 4. The results from the analysis are discussed in Sect. 5. We end the paper with a conclusion in Sect. 6.

\section{KOINet data}

In order to organise the KOINet observations we calculated transit time predictions from the Kepler observations as described in Sect. 2.5 of von Essen et al. (2018). In the case of Kepler-82b, a linear plus sine function was fitted to predict future times of transit. For Kepler-82c we provided two different predictions. One coming from a sine plus linear fit, and one from fitting a parabolic function as a turnover to the sine curve was not measured by the Kepler observations. The low precision in the transit time predictions of Kepler-82c in particular led to only a small fraction of KOINet Kepler-82 light curves with transits included. Between 2014 and 2018 eleven light curves of Kepler- 82 were obtained, while only three of them show transit signals of Kepler-82b/c.

Table 1 lists the main characteristics of the data presented in this paper, such as the observing telescope and the observation dates the precision of the data, the total duration of the observation, and the transit coverage. To increase the photometric precision of the collected data, we have, when possible, slightly defocused the telescopes (Kjeldsen \& Frandsen 1992; Southworth et al. 2009). Below is a brief description of the main characteristics of each of the telescopes involved in this work.

The Apache Point Observatory hosts the Astrophysical Research Consortium $3.5 \mathrm{~m}$ telescope (henceforth "ARC $3.5 \mathrm{~m}$ "), and is located in New Mexico, United States of America. The photodynamical analysis of Kepler- 82 presented here includes one light curve taken with the ARC $3.5 \mathrm{~m}$ during our first observing campaign in 2014.

The $2.5 \mathrm{~m}$ Nordic Optical Telescope (NOT $2.5 \mathrm{~m}$ ) is located at the Observatorio Roque de los Muchachos in La Palma, Spain. Currently, telescope time for KOINet is assigned via a large (three-years) program. Here, we present two light curves taken between the fourth and fifth observing seasons.

The $80 \mathrm{~cm}$ telescope of the Instituto de Astrofísica de Canarias (IAC $0.8 \mathrm{~m}$ ) is located at the Observatorio del Teide, in the Canary Islands, Spain. The one transit light curve obtained in the first season of KOINet suffered from technical difficulties during the night. For this reason the resulting science frames were corrupted and, thus, it was impossible for us to properly reduce them.

The Oskar Lühning Telescope (OLT 1.2 m) has a $1.2 \mathrm{~m}$ aperture diameter and is located at the Hamburger Observatory in Hamburg, Germany. Kepler-82 was observed for one night in the first season of KOINet with OLT $1.2 \mathrm{~m}$. Unfortunately, the observation taken in 2014 suffered from technical difficulties.

The Telescopi Joan Oró is a fully robotic $80 \mathrm{~cm}$ telescope (TJO $0.8 \mathrm{~m}$ ) located at the Observatori Astronomic del Montsec, in the north-east of Spain. The parabolic prediction of Kepler-82c was chosen as transit time for an observation. The obtained observation contains only off-transit data.

The fully robotic $2 \mathrm{~m}$ Liverpool telescope (LIV $2 \mathrm{~m}$; Steele et al. 2004) is located at the Observatorio Roque de los Muchachos and is owned and operated by Liverpool John Moores 
J. Freudenthal et al.: Kepler Object of Interest Network. III.

Table 1. Characteristics of collected ground-based transit light curves of Kepler-82b/c, collected through KOINet.

\begin{tabular}{cccccccc}
\hline \hline $\begin{array}{c}\text { Date } \\
\text { yyyy.mm.dd }\end{array}$ & Planet & Telescope & $\begin{array}{c}\sigma_{\text {res }} \\
(\mathrm{ppt})\end{array}$ & $N$ & $\begin{array}{c}\text { CAD } \\
(\mathrm{s})\end{array}$ & $\begin{array}{c}T_{\text {tot }} \\
(\mathrm{h})\end{array}$ & TC \\
\hline 2014.09 .04 & $\mathrm{~b}$ & ARC 3.5 m & 1.1 & 235 & 60 & 4.6 & - - B E O \\
2017.07 .08 & $\mathrm{c}$ & NOT 2.5 m & 1.4 & 111 & 193 & 4.2 & O I - - \\
2018.07 .05 & $\mathrm{c}$ & NOT 2.5 m & 1.2 & 112 & 179 & 5.7 & O I - - \\
2015.05 .26 & $\mathrm{~b}$ & SAO 6.5 m & 0.3 & 32 & 68 & 1.1 & Only off-transit data \\
2015.05 .28 & $\mathrm{c}$ & CAHA 2.2 m & 10.8 & 75 & 86 & 1.9 & Only off-transit data \\
2015.07 .19 & $\mathrm{c}$ & CAHA 3.5 m & 4.4 & 292 & 102 & 6.8 & Parabolic solution was chosen \\
& $\mathrm{c}$ & LIV 2 m & 1.5 & 112 & 90 & 3.0 & Parabolic solution was chosen \\
2018.07 .05 & $\mathrm{c}$ & TJO 0.8 m & 4.4 & 163 & 69 & 4.4 & Parabolic solution was chosen \\
\hline 2014.07 .23 & KRYO 1.2 m & 3.7 & 242 & 83 & 5.8 & Only off-transit data \\
\hline & IAC 0.8 m & & & & Corrupted data \\
\hline
\end{tabular}

Notes. From left to right: the date on which the observations were carried out, in years, months and days; the planet the transit belongs to; an acronym for the telescope used to perform the observations; the precision of the data in parts-per-thousand (ppt), $\sigma_{\text {res }}$; the number of frames acquired during the night, $N$; the cadence of the data considering the readout time in seconds, CAD; the total duration of the observations in hours, $T_{\text {tot }}$; the transit coverage, TC. The letter code to specify the transit coverage during each observation is the following: O: out of transit, before ingress. I: ingress. B: flat bottom. E: egress. O: out of transit, after egress.

University. During the second season of KOINet a transit time predicted from parabolic TTVs was chosen for an observation. The resulting light curve does not contain a transit.

The Centro Astronómico Hispano-Alemán hosts, among others, a $2.2 \mathrm{~m}$ and a $3.5 \mathrm{~m}$ telescope ("CAHA $2.2 \mathrm{~m}$ " and "CAHA 3.5 m"). An observation was taken with each telescope. No transit is present in the light curves.

The MMT observatory, a joint venture of the Smithsonian Institution and the University of Arizona, is located on the summit of Mt. Hopkins in south-eastern Arizona, USA. The telescope has a collecting area of $6.5 \mathrm{~m}(\mathrm{SAO} 6.5 \mathrm{~m})$. The data collected with this telescope were of sub-millimagnitude precision, but taken outside transit due to bad scheduling decisions.

The National Observatory of Athens hosts the $1.2 \mathrm{~m}$ Cassegrain telescope of the Astronomical Station Kryoneri (KRYO $1.2 \mathrm{~m}$ ). For the last $40 \mathrm{yr}$ the telescope has been operational, with an extensive upgrade taking place in 2016. Data collected with this telescope were of good quality, however taken outside transit.

All collected observations underwent the KOINet reduction pipeline, and a preliminary analysis for deriving reliable errorbars and the detrending components. This process is described in von Essen et al. (2018) and Freudenthal et al. (2018).

\section{The photodynamical model}

For the KOINet data analysis we developed a simultaneous transit light curve model for all observations of each system that takes the system dynamics into account. This allows us to determine the planetary masses in addition to the transit parameters. A full description of our photodynamical model can be found in Freudenthal et al. (2018). Briefly, we combine a numerical integration of the whole system over the time span of observations, and from the output sky positions (projected distance of each planet to the star) we calculate the transit light curve. We use a second-order mixed-variable symplectic (MVS) algorithm to perform the numerical integration as implemented in our python-wrapper for mercury6 (Chambers 1999). The integrator is complemented by first-order post-Newtonian correction (Kidder 1995), and we correct the individual times for the lighttravel-time effect for each planet. From the numerical integration of the system we extract the planet-to-star centre distances to calculate the light curve through the transit model of Mandel \& Agol (2002). Here we use the occultquad routine with the quadratic limb-darkening law implemented.

As in Freudenthal et al. (2018), the numerical integration is done on a coarse grid, and only in the vicinity of transits is the integration refined with a time step of $0.01 \mathrm{~d}$. The coarse grid is optimised to give the shortest possible computation time with sufficient accuracy. For this system a time step of a hundredtwentieth of the period of the innermost included planet was used. For long-cadence data we take the finite integration time into account (Kipping 2010). Hence, we compute the transit light curve with a time step of $\sim 1 \mathrm{~min}$ and rebin it to the cadence of the data points.

Our photodynamical model is coupled to the Markov chain Monte Carlo (MCMC) emcee3 algorithm (Foreman-Mackey et al. 2013). All fitting parameters have uniform priors with broad boundaries chosen to avoid non-physical results. A detailed description of the model parameters can be found in Freudenthal et al. (2018). To summarise, the model requires the mass, $m$, and the radius $R$ of the central star, as well as the two quadratic limb darkening coefficients, $c_{1}$ and $c_{2}$, that reflect the wavelength response of the optical setup of each telescope per instrument, and per planet, $p(p \in\{b, c, f\}$ from Sect. 4 and for example in the Tables 2 and A.2) the parameters are described below.

A mass ratio is needed. For the innermost planet the ratio to the central star, $m_{1} / m_{\mathrm{S}}$, is taken and for all other planets the ratio to the next inner one, $m_{\mathrm{p}} / m_{\mathrm{p}^{\prime}}$. Secondly, a parameter to calculate the semi-major axis, $a$, is needed. In the case of transiting planets it is calculated from the mean period, $P_{\mathrm{p}}$ and as a free parameter a correction factor, $a_{\mathrm{p}, \mathrm{corr}}$ :

$$
a_{\mathrm{p}}=\left(\frac{P_{\mathrm{p}}^{2} G\left(m_{\mathrm{S}}+m_{\mathrm{p}}\right)}{4 \pi^{2}}\right)^{1 / 3} \times a_{\mathrm{p}, \mathrm{corr}},
$$

with the gravitational constant, $G$. We fitted a linear ephemeris $T=\Delta T_{\mathrm{p}, 0}+P_{\mathrm{p}} \times n$ to the transit times, $T$, giving us the mean period $P_{\mathrm{p}}$ and an offset $\Delta T_{\mathrm{p}, 0}$. For non-transiting planets the semi-major axis is calculated from the period given by a period ratio to the next inner planet. Furthermore, the eccentricity, $e_{\mathrm{p}}$, 
is needed. The orbital angles, inclination, $i_{\mathrm{p}}$, argument of the periastron, $\omega_{\mathrm{p}}$, and the longitude of the ascending node, $\Omega_{\mathrm{p}}$, are needed. Whereas the latter is fixed to zero for the innermost planet, the other values are given relative to the innermost planet. The instantaneous position of the planets at a given reference time needs to be defined. We take the mean anomaly, $M_{\mathrm{p}}$, as measurement for the position of each planet. This angle is calculated from the mean period, $P_{\mathrm{p}}$, as well as the offset, $\Delta T_{\mathrm{p}, 0}$. As a free parameter, we have an addition to this derived mean anomaly, $M_{\mathrm{p}, \mathrm{corr}}$ :

$M_{\mathrm{p}}=M_{\mathrm{p}, \text { Kepler }}-\frac{2 \pi}{P_{\mathrm{p}}} \Delta T_{\mathrm{p}, 0}+M_{\mathrm{p}, \mathrm{corr}}$

with the mean anomaly at transit time calculated for a Kepler orbit from the argument of periastron and eccentricity, $M_{\mathrm{p}, \text { Kepler, }}$, and the second term is giving the difference between the mean anomaly at transit time and the mean anomaly at the starting time of the integration. That means the free parameter $M_{\mathrm{p} \text {,corr }}$ is giving the correction from a pure Keplerian orbit due to the interaction with the other planets. Lastly, The planet-tostar radius ratio, $R_{\mathrm{p}} / R_{\mathrm{S}}$, only for transiting planets needs to be given.

We treated Kepler data and ground based observations of KOINet as the description in Freudenthal et al. (2018). From Kepler photometry we extracted the transit duration symmetrically around each transit mid point four times. To account for intrinsic stellar photometric variability we normalised each transit light curve dividing it by a time dependent second-order polynomial optimised on the off-transit data points. The coefficients of this parabola are derived through a simple least-squares minimisation routine. As previously mentioned, for long-cadence data, the photodynamical light curve model is oversampled by a factor of 30 and rebinned to the actual data points. This procedure is not necessary for short-cadence data. The high signal-to-noise ratio $(\mathrm{S} / \mathrm{N})$ of Kepler data allows us to include the quadratic limb darkening coefficients into our free parameters set. This allows for a more realistic inclination and star and planetary radii determination due to the good constrained transit shape.

Due to the lower $\mathrm{S} / \mathrm{N}$ of the ground-based data, we fixed the quadratic limb darkening coefficients to values which are derived as described in von Essen et al. (2013) from stellar parameters for the Johnson-Cousins $R$-band filter, which we used for all of our observations. For stellar parameters closely matching the ones of Kepler-82 (Petigura et al. 2017), the derived limb darkening coefficients are $c_{1}=0.52$ and $c_{2}=0.14$. The best-matching coefficients of the detrending components, derived during the first data analysis (in Sect. 2), for each ground-based observation are calculated as a linear combination at each call of the photodynamical model.

\section{Dynamical analysis of Kepler-82}

In the following sections we outline the detection of a fifth, non-transiting planet in the Kepler-82 system, which is required to explain the available data. We call the planet Kepler-82f hereafter.

In this work we analyse the transit light curves of the outer two planets of Kepler-82, b and c. These planets have a period ratio close to the 2:1 resonance. The inner two, $\mathrm{d}$ and e, show no strong TTV amplitudes and especially no frequencies due to interaction with the outer two (Ofir et al. 2018). In a first step we determined the transit times from long-cadence Kepler data with the procedure described in Sect. 4.1 of von Essen et al. (2018). In addition to the near resonant interaction with Kepler-82b, the transit times of Kepler-82c show a strong "chopping" component, which is visible by a sudden jump in the transit time following every three consecutive transits which show drifting transit times. The period of chopping is controlled by the times between conjunctions of planet $\mathrm{c}$ and the fifth planet, given by the synodic period

$P_{\text {syn }}=\left|\frac{1}{P_{\text {out }}}-\frac{1}{P_{\text {in }}}\right|^{-1}$.

Since the jump in chopping is seen every three transits of planet $\mathrm{c}$, this indicates that the synodic period is either $3 \times P_{\mathrm{c}}$ or $3 / 2 \times P_{\mathrm{c}}$, which would give a dependency of the acceleration and the deceleration during the orbits of the inner planet from three times its period. These synodic periods can be created by an outer planet near the $3: 2$ or $3: 1$ resonance with planet $c$. Based on the synodic period of planet c, an inner planet near the $3: 4$ or $3: 5$ resonance would also be possible; however, such a planet would be near a 3:2 or 6:5 resonance with Kepler-82b, and would then induce a strong signal in its TTVs. Such a TTV signal is not measured; hence the fifth planet must orbit exterior to planet $\mathrm{c}$.

For this reason we optimised the parameters of the two outer unknown planet configurations (from now on the 3:2:1 and 6:2:1 resonance models, for convenience we skip the more accurate notation of the planets being near resonant) in a photodynamical model applied to the Kepler long-cadence (quarters 1-6) and short-cadence (quarters 7-17) data. From the Kepler data alone, both of the resonance models show the same probability. The prediction for the transit times, however, start to diverge rapidly after the Kepler mission terminates, as visualised in Fig. 1. The figure shows the transit times with a linear ephemeris subtracted (observed minus calculated, thus henceforth, O-C diagram) of Kepler- $82 b$ at the top and of Kepler-82c at the bottom. For Kepler-82b the models start to differ within $3 \sigma$ by the end of 2015 and for Kepler-82c by mid 2014. The three KOINet transit light curves (plotted in Fig. 2; in the $\mathrm{O}-\mathrm{C}$ diagram the transit times are indicated in red) show a clear preference for the 3:2:1 resonance model. In addition, the latest KOINet observation where no transit is measured clearly contradicts the $6: 2: 1$ resonance model prediction.

On this account we re-optimised the 3:2:1 resonance model parameters to the Kepler data complemented by the three KOINet transit light curves. The resulting planetary and stellar parameters from this fit can be found in Table 2. Table A.2 lists the planetary and stellar parameters from all model optimisation done in this work. The tables shows from top to bottom the modelled and derived values of Kepler-82b, Kepler-82c, the new planet, Kepler-82f, and the central star. The osculating orbital elements are given at the reference time BJD $=2454933.0$, 100 days later than the standard Kepler reference time (BKJD).

For comparison we also optimised the transiting 2-planet system (2:1 resonance model) on the Kepler long- and shortcadence data. The results are listed as well and presented in the $\mathrm{O}-\mathrm{C}$ diagram (Fig. 1) as grey areas.

\subsection{Details of optimisation}

We initially optimised the different planetary system models (described later in this section) on the transit times, fixing all transit shape determining parameters to narrow the parameter space for the photodynamical analysis. We used the median 


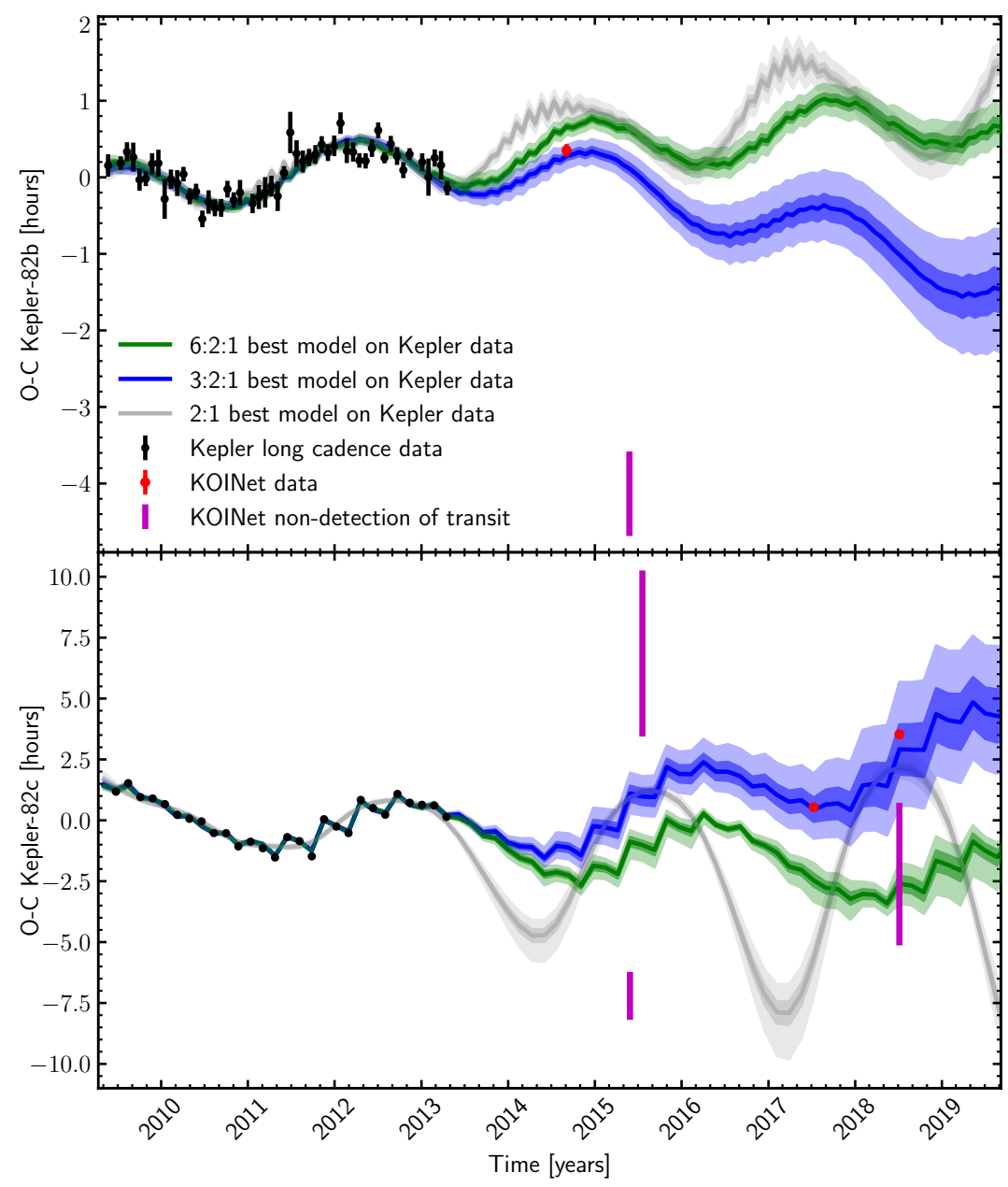

Fig. 1. O-C diagrams of Kepler-82b at the top and Kepler-82c at the bottom with transit times from modelling the transits individually. The black points refer to the transit data from the Kepler telescope. The red points are the individual transit times from the new KOINet observations (plotted in Fig. 2). The violet lines show observed epochs but with nondetections of transit. The green area indicates the $99.7 \%$ (light) and the $68.3 \%$ (dark) confidence interval of the $6: 2: 1$ resonance model optimised on Kepler long- and short-cadence data only, derived from 1000 randomly chosen models out of the MCMC posterior distribution; the green line is the median. The blue areas indicate the 3:2:1 resonance model solution. The grey areas present the 2:1 resonance model. values and the $3 \sigma$ interval of this analysis for a Gaussian random choice of starting parameter sets. The parameters describing the transit shape - the inclination, limb darkening coefficients and planet and star radii - are taken from the individual transit fits.

We fixed the stellar mass to its literature value of $m_{\mathrm{S}}=0.91 M_{\odot}$ (Johnson et al. 2017) during the TTV and the photodynamical analysis. The uncertainty on the stellar mass, $\sigma_{m_{\mathrm{S}}}=0.03 M_{\odot}$, is applied to the derived parameters that depend on it via error propagation. In particular this affects the planetary masses, semi-major axes, and periods.

Optimising a linear ephemeris to the Kepler transit times of Kepler-82b/c, we obtained the offsets $\Delta T_{\mathrm{b}, 0}=41.23683 \mathrm{~d}$ and $\Delta T_{\mathrm{c}, 0}=22.52550 \mathrm{~d}$ as intercepts, and the mean periods $P_{\mathrm{b}}=$ $26.44404770 \mathrm{~d}$ and $P_{\mathrm{c}}=51.53912652 \mathrm{~d}$ as slopes. The offsets and mean periods are used for the determination of the semimajor axes and the mean anomalies, as described previously in Sect. 3.

The properties of all of the photodynamical model optimisation procedures on the transit light curves are given in Table 3 . Listed are the parameters as follows. In the first row the number of walkers used for extracting the final results are given. We initialised with more walkers; however, a variable number of walkers ended in higher $\chi^{2}$ minima. Next, the number of iterations we obtained per walker are given, followed by the number of iterations we used as initial burn-in. From the MCMC posterior distribution we calculated the autocorrelation length according to Goodman \& Weare (2010), but averaging over the autocorrelation function per walker instead of averaging directly over the walker values, as discussed in the blog by Daniel Foreman-Mackey ${ }^{2}$. The given autocorrelation length allows us to derive the effective number of individual samples. The last two rows contain the degree of freedom (d.o.f.) of the optimisation and the best reduced $\chi^{2}$ value. We note a significant deviation from one in the reduced $\chi^{2}$ values which is unexpected considering the high dof numbers. For this reason, we quadratically add a systematic error of $\sqrt{\chi_{\text {red }}^{2}}-1 \sim 10 \%$ to the model parameter uncertainties in Tables 2 and A.2.

While optimising the $3: 2: 1$ resonance model we realised that we could actually derive the entire orbit of the non-transiting planet. By this we mean that we could constrain the inclination - which avoids transit - and the other orbital angles: the longitude of periastron, the longitude of ascending node, and the mean anomaly. We found two different configurations with $i_{\mathrm{b}}$ constrained to below $90^{\circ}$. The first has $i_{\mathrm{c}}<90^{\circ}$ and $i_{\mathrm{f}}>90^{\circ}$ (henceforth configuration I), the second is the opposite with $i_{\mathrm{c}}>90^{\circ}$ and $i_{\mathrm{f}}<90^{\circ}$ (configuration II). The configurations are visualised in Fig. 3, where the impact parameter of the planets is plotted against the distance to the star. The values and uncertainties are derived from 1000 randomly chosen results from

2 https://dfm.io/posts/autocorr/ 

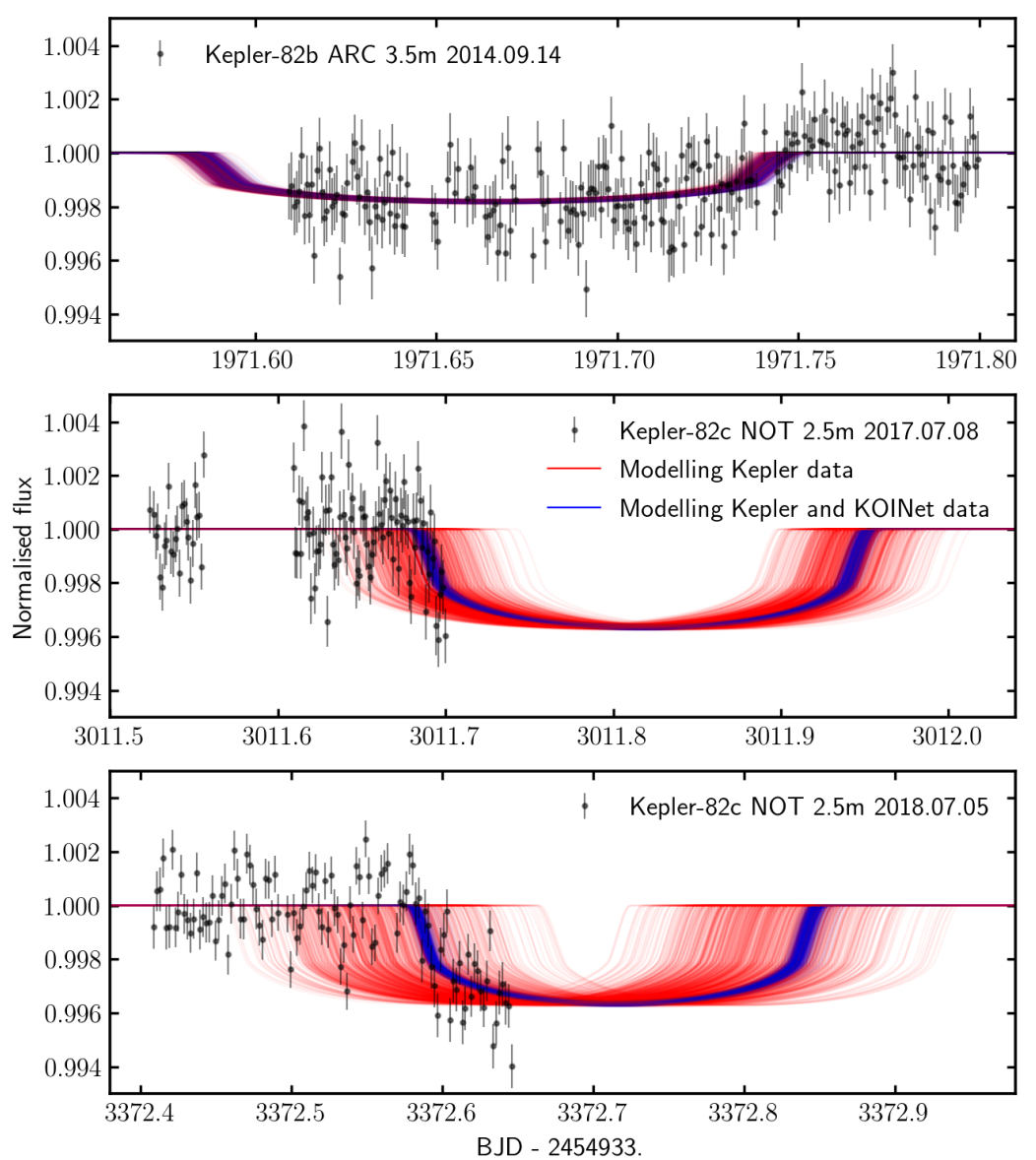

Fig. 2. KOINet transit light curves of Kepler-82. The three transit light curves (black) are overplotted with 1000 random models of the 3:2:1 resonance optimised using Kepler long- and short-cadence data (red) and including these KOINet observations (blue). modelling the Kepler and KOINet data in configuration I in red and in configuration II in blue. Both configurations have the same probability and are equivalent in all other parameters. This means that the transit time predictions and shape are the same for both configurations. The other two configurations, with either both planets having inclinations below $90^{\circ}$ or both above $90^{\circ}$, are not chosen by the MCMC optimisation, although allowed and included in the starting positions of the walkers. We modelled both configurations individually with the same number of iterations and combined all resulting walkers to extract the results. Given that the KOINet transit times are located at the 3:2:1 resonance model predictions, we optimised this model on these light curves together with the Kepler data again in both of the configurations.

Following this detection we also set the inclination of the non-transiting planet in the 6:2:1 resonance model as a free parameter. In this case the inclination did not avoid the transit region, though it spans a large area where the majority of solutions is in the non-transiting region (about 88\% in a conservative calculation of the impact parameter $b$ ). Nonetheless, we inspected the Kepler data for these transits. Based on the mass ratio to Kepler- $82 \mathrm{c}$ of $23_{-1.9}^{+2.5}$ we can expect transits of larger depths compared with the other system's planets. Such transits are not detected.

\subsection{Results}

Along with the optimised parameters listed in Tables 2 and A.2 we display the KOINet transit light curves in Fig. 2 in black.
These are overplotted with 1000 model solutions randomly chosen from the MCMC posterior distribution from analysing only Kepler data in red, and including these KOINet transit light curves in blue with the 3:2:1 resonance model. Similar to the $\mathrm{O}-\mathrm{C}$ plot in Fig. 1 we show the TTV behaviour for the 3:2:1 resonance model optimised on all available transit light curves in comparison to the optimisation on Kepler data only in Fig. A.1. Including the KOINet transit observations led to a narrowing of the transit time predictions of Kepler-82c (visible in the Figs. 2 and A.1) and the shrinkage of the mass uncertainties of Kepler-82b (see Table A.2). The transit time predictions for the next fifteen years are listed in Table A.1. Finally, the parameter correlations are visualised in a corner plot in Fig. A.3.

\section{Discussion}

The most prominent signal in the TTVs of the Kepler- $82 \mathrm{~b} / \mathrm{c}$ system is the dynamical interaction with each other due to its near 2:1 resonance configuration. Xie (2013) calculated nominal masses from the amplitudes of these TTVs and a derived stellar mass from $\log g$ and $R_{\mathrm{S}}$ under the assumption of a 2interacting-planet system. Their derived masses for Kepler-82b/c are $m_{\mathrm{b}}=87.0_{-22.4}^{+251.8} M_{\oplus}$ and $m_{\mathrm{c}}=19.1_{-4.9}^{+55.5} M_{\oplus}$, respectively. Additionally, they derive the planetary radii from single transit fitting to $R_{\mathrm{b}}=4.00 \pm 1.82 R_{\oplus}$ and $R_{\mathrm{c}}=5.35 \pm 2.44 R_{\oplus}$. With these values they propose a density ratio of $\sim 10$ for the planets. In an initial model we tested this 2-planet system with our photodynamical analysis. We found planetary masses and radii 
Table 2. Planetary and Stellar parameters from photodynamical analysis of the 3:2:1 resonance model on Kepler data and the three KOINet transit light curves.

\begin{tabular}{|c|c|}
\hline Parameter & Values \\
\hline \multicolumn{2}{|l|}{ Kepler- $82 b$} \\
\hline$m_{\mathrm{b}} / m_{\mathrm{S}}$ & $0.0000401_{-0.0000025}^{+0.0000028}$ \\
\hline$m_{\mathrm{b}}^{*}\left(M_{\oplus}\right)$ & $12.15_{-0.87}^{+0.96}$ \\
\hline$a_{\mathrm{b}, \mathrm{corr}}$ & $0.9999606_{-0.0000041}^{+0.000042}$ \\
\hline$a_{\mathrm{b}}^{*}(\mathrm{AU})$ & $0.1683 \pm 0.0020$ \\
\hline$P_{\mathrm{b}}^{*}(\mathrm{~d})$ & $26.44 \pm 0.48$ \\
\hline$e_{\mathrm{b}}$ & $0.0033_{-0.0017}^{+0.0019}$ \\
\hline$i_{\mathrm{b}}\left({ }^{\circ}\right)$ & $89.052_{-0.096}^{+0.049}$ \\
\hline$\Omega_{\mathrm{b}}\left({ }^{\circ}\right)$ & 0 (fixed) \\
\hline$\omega_{\mathrm{b}}\left({ }^{\circ}\right)$ & $236_{-26}^{+23}$ \\
\hline$M_{\mathrm{b}, \mathrm{corr}}\left({ }^{\circ}\right)$ & $-0.025_{-0.020}^{+0.019}$ \\
\hline$M_{\mathrm{b}}^{*}\left({ }^{\circ}\right)$ & $13_{-23}^{+26}$ \\
\hline$R_{\mathrm{b}} / R_{\mathrm{S}}$ & $0.04159_{-0.00045}^{+0.00049}$ \\
\hline$R_{\mathrm{b}}^{*}\left(R_{\oplus}\right)$ & $4.07_{-0.10}^{+0.24}$ \\
\hline$\rho_{\mathrm{b}}^{*}\left(\mathrm{~g} \mathrm{~cm}^{-3}\right)$ & $0.98_{-0.16}^{+0.11}$ \\
\hline \multicolumn{2}{|l|}{ Kepler-82c } \\
\hline$m_{\mathrm{c}} / m_{\mathrm{b}}$ & $1.14_{-0.13}^{+0.14}$ \\
\hline$m_{\mathrm{c}}^{*}\left(M_{\oplus}\right)$ & $13.9_{-1.2}^{+1.3}$ \\
\hline$a_{\mathrm{c}, \mathrm{corr}}$ & $0.999947_{-0.000019}^{+0.000018}$ \\
\hline$a_{\mathrm{c}}^{*}(\mathrm{AU})$ & $0.2626 \pm 0.0032$ \\
\hline$P_{\mathrm{c}}^{*}(\mathrm{~d})$ & $51.54 \pm 0.94$ \\
\hline$e_{c}$ & $0.0070_{-0.0018}^{+0.0016}$ \\
\hline$i_{\mathrm{c}}\left({ }^{\circ}\right)$ config. I & $90.15_{-0.22}^{+0.18}$ \\
\hline$i_{\mathrm{c}}\left({ }^{\circ}\right)$ config. II & $89.78_{-0.15}^{+0.17}$ \\
\hline$\Omega_{\mathrm{c}}\left({ }^{\circ}\right)$ & $1.6 \pm 2.1$ \\
\hline$\omega_{\mathrm{c}}\left({ }^{\circ}\right)$ & $162_{-20}^{+12}$ \\
\hline$M_{\mathrm{c}, \text { corr }}\left({ }^{\circ}\right)$ & $-0.507 \pm 0.020$ \\
\hline$M_{\mathrm{c}}^{*}\left({ }^{\circ}\right)$ & $131_{-12}^{+20}$ \\
\hline$R_{\mathrm{c}} / R_{\mathrm{S}}$ & $0.05453_{-0.00053}^{+0.00068}$ \\
\hline$R_{\mathrm{c}}^{*}\left(R_{\oplus}\right)$ & $5.34_{-0.13}^{+0.32}$ \\
\hline$\rho_{\mathrm{c}}^{*}\left(\mathrm{~g} \mathrm{~cm}^{-3}\right)$ & $0.494_{-0.083}^{+0.070}$ \\
\hline \multicolumn{2}{|l|}{ Kepler-82f } \\
\hline$m_{\mathrm{f}} / m_{\mathrm{c}}$ & $1.50_{-0.13}^{+0.16}$ \\
\hline$m_{\mathrm{f}}^{*}\left(M_{\oplus}\right)$ & $20.9 \pm 1.0$ \\
\hline$P_{\mathrm{f}} / P_{\mathrm{c}}$ & $1.46940_{-0.00022}^{+0.00023}$ \\
\hline$P_{\mathrm{f}}^{*}(\mathrm{~d})$ & $75.732 \pm 0.012$ \\
\hline$a_{\mathrm{f}}^{*}(\mathrm{AU})$ & $0.3395 \pm 0.0041$ \\
\hline$e_{\mathrm{f}}$ & $0.0014_{-0.0010}^{+0.0018}$ \\
\hline$i_{\mathrm{f}}\left({ }^{\circ}\right)$ config. I & $86.30 \pm 0.56$ \\
\hline$i_{\mathrm{f}}\left({ }^{\circ}\right)$ config. II & $93.62_{-0.72}^{+0.56}$ \\
\hline$\Omega_{\mathrm{f}}\left({ }^{\circ}\right)$ & $1.6_{-2.1}^{+2.2}$ \\
\hline$\omega_{\mathrm{f}}\left({ }^{\circ}\right)$ & $62_{-47}^{+70}$ \\
\hline$M_{\mathrm{f}}\left({ }^{\circ}\right)$ & $125_{-70}^{+47}$ \\
\hline \multicolumn{2}{|l|}{ Kepler-82 } \\
\hline$m_{\mathrm{S}}\left(M_{\odot}\right)$ & $0.91 \pm 0.03$ (fixed, Johnson et al. 2017) \\
\hline$R_{\mathrm{S}}\left(R_{\odot}\right)$ & $0.898_{-0.018}^{+0.042}$ \\
\hline$\rho_{\mathrm{S}}^{*}\left(\mathrm{~g} \mathrm{~cm}^{-3}\right)$ & $1.77_{-0.23}^{+0.11}$ \\
\hline$c_{1, \text { Kepler }}$ & $0.522_{-0.075}^{+0.054}$ \\
\hline$c_{2, \text { Kepler }}$ & $0.12_{-0.09}^{+0.14}$ \\
\hline
\end{tabular}

Notes. Listed are the median values and $68.26 \%$ confidence interval from the MCMC posterior distribution. The osculating orbital elements are given at a reference time, BJD $=2454933.0{ }^{\left({ }^{(*)} \text { Derived, not fitted }\right.}$ parameters. with much smaller uncertainties (see Table A.2) that agree within their errorbars with the values calculated by Xie (2013). The density ratio of our result is even higher with $\rho_{\mathrm{b}} / \rho_{\mathrm{c}} \sim 14$.

The stellar parameters of this analysis show significant deviations from literature values that are derived by spectroscopic observations. The stellar radius with $R_{\mathrm{S}}=1.186_{-0.077}^{+0.074}$ is more than $1 \sigma$ higher than the measurement by Johnson et al. (2017) $\left(R_{\mathrm{S}}=0.99_{-0.08}^{+0.10} R_{\odot}\right)$ and the quadratic limb darkening coefficients calculated by Claret \& Bloemen (2011; ATLAS model) to $c_{1}=0.4695$ and $c_{2}=0.2240$ do not fall within the modelled values $\left(c_{1}=0.31_{-0.17}^{+0.20}, c_{2}=0.66_{-0.32}^{+0.24}\right)$.

These stellar parameters as well as the planetary masses, and with these the densities, become more plausible in their values when including a third planet in the dynamical analysis. The signal of such a planet is clearly visible in the TTVs of Kepler-82c as a jump every three consecutive transits (see Fig. 1). As explained in Sect. 3, two different configurations of a threeplanet system can explain this chopping effect in the Kepler data. Both of these include another outer non-transiting planet, near the $3: 1$ or 3:2 period resonance to Kepler-82c. Including either of these planets dramatically reduces the mass of Kepler- $82 \mathrm{~b}$, and thus also reduces the ratio of the density Kepler- $82 \mathrm{~b}$ to c. Both system models are very similar in probability for Kepler data, the 6:2:1 resonance model has a slightly higher $\chi_{\text {red }}^{2}$ than the 3:2:1 resonance system. With KOINet data we were able to distinguish between these two models. The detected transits fall at the 3:2:1 model prediction, and one of the observations where no transit is observed precludes the 6:2:1 model predicted transit time. In the following we refer to the 3:2:1 resonance model solution on Kepler and KOINet data when not differently specified.

The density ratio of the resulting Kepler- $82 \mathrm{~b} / \mathrm{c}$ planets reduces to a factor of $\sim 2$. Such a ratio is no longer very unusual; the values are discussed in the context of the literature below by visualising them in a mass-radius diagram. The density of the new planet can not be determined as, due to the lack of transits, the radius is not measurable. In addition, the stellar radius and the limb darkening values fit in with the literature values within $1 \sigma$-uncertainty.

At the same time the predicted RV signal reduces from an amplitude of $\sim 50 \mathrm{~m} \mathrm{~s}^{-1}$ for the 2-planets system to about $\sim 7.5 \mathrm{~m} \mathrm{~s}^{-1}$ for the 3-planets system near 3:2:1 resonance. With Kepler- 82 being a relatively faint star $(\mathrm{Kp}=15.158)$, such a signal is not measurable with current instruments.

\subsection{Previously proposed planets}

Bovaird et al. (2015) predicted two additional planets in the Kepler-82 system with periods of $11.8 \pm 2.0$ days and $120 \pm$ 20 days based on the Titius-Bode relation. Neither the new planet proposed here near the 3:2 resonance to Kepler-82c, nor the less viable option with a planet near the $3: 1$ resonance, matches the position of one of the predicted planets. The predicted outer planet is in between the two possibilities within $3 \sigma$ distance to each of them.

\subsection{Dynamical stability}

Subsequent to the photodynamical analysis we tested the dynamical stability of the modelled systems. With the same integrator, the second-order mixed-variable symplectic algorithm implemented in the mercury6 package by Chambers (1999), we extend the numerical simulation of the best found solution for each system configuration to $10 \mathrm{Gyr}$. For this application the post-Newtonian correction (Kidder 1995) was implemented as well. The integration is done with a time step size of 1 day 
A\&A 628, A108 (2019)

Table 3. Properties of the optimisation of different models paired with different data sets.

\begin{tabular}{lcccc}
\hline \hline Parameter & Kepler data & Kepler data & Kepler data & $\begin{array}{c}\text { Kepler \& } \\
\text { KOINet data }\end{array}$ \\
& $2: 1$ & $6: 2: 1$ & $3: 2: 1$ & $3: 2: 1$ \\
\hline Walkers & 56 & 59 & 91 & 86 \\
Iterations per walker & 20000 & 75000 & 100000 & 175000 \\
Iterations burn-in per walker & 10000 & 25000 & 25000 & 25000 \\
Autocorrelation length & 1136 & 11498 & 14595 & 22422 \\
Independent samples in total & 986 & 385 & 624 & 671 \\
Degrees of freedom & 63372 & 63365 & 63365 & 63823 \\
Best $\chi_{\text {red }}^{2}$ & 1.239 & 1.225 & 1.224 & 1.227 \\
\hline
\end{tabular}

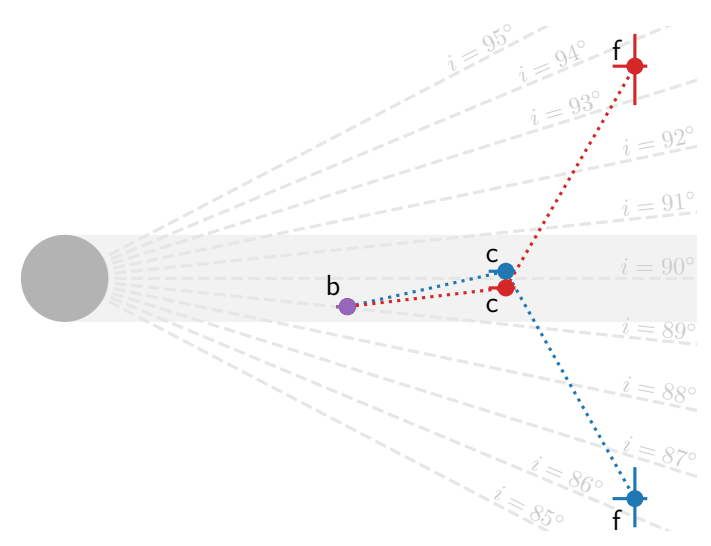

Fig. 3. Configurations of the Kepler- 82 system. With the star in grey on the left side and the observer on the right side this shows the two different configurations: $\mathrm{b}$ in violet has the same position in both, $\mathrm{c}$ and $\mathrm{f}$ in red shows configuration I and in blue configuration II. The grey area indicates the region of impact parameters below one. The distances are not true to scale with the stellar radius, therefore a few inclination values are indicated as dashed grey lines. A similar plot with true scales can be found in Fig. A.2.

which is roughly a twentieth of the innermost planet considered in our analysis (Kepler-82b). This gives a good compromise between a sufficient sampling for small integration errors and a reasonable computation time. We tested the stability of the $2: 1$ resonance 2 planets solution, the $6: 2: 1$ resonance system as well as the 3:2:1 resonance 3 planets model that is preferred by the KOINet data. All of these system configurations survived the $10 \mathrm{Gyr}$ integration; only the 2-planet system showed chaotic parameter evolution. A closer inspection of the 6:2:1 resonance system long-term behaviour showed that given this model we are observing the transiting planets $\mathrm{b}$ and $\mathrm{c}$ at a minimum in periodically changing eccentricities. The values are ranging in roughly $e_{\mathrm{b}}=0.002-0.08$ and $e_{\mathrm{c}}=0.004-0.06$. The probability for the planets to be in this minimum at observation time is below $10 \%$, making this scenario even less likely.

Another indication for stability is the planets to be near resonant, but not in resonance. We checked that the modelled planets are not in resonance through calculating the resonant angles (Morbidelli 2002), as well as the Laplace resonant angle. All angles are circulating and do not librate, which would be the sign for the planets to be in resonance.

\subsection{TTV frequencies}

The frequencies in the TTVs of the Kepler- 82 system were analysed by Ofir et al. (2018). In the TTVs of Kepler-82b they A108, page 8 of 17
Table 4. Comparison between TTV inducing frequencies calculated from periods of the system solution to measured TTV frequencies by Ofir et al. (2018).

\begin{tabular}{lrlr}
\hline \hline Planets & \multicolumn{1}{l}{$f_{\text {calc }}\left(10^{-4} \mathrm{~d}^{-1}\right)$} & TTV matching & \multicolumn{1}{l}{$f_{\text {Ofir }}\left(10^{-4} \mathrm{~d}^{-1}\right)$} \\
\hline \multicolumn{2}{l}{ Super frequencies } \\
b and c & $9.86 \pm 0.22$ & Main peak in b & \\
b and f & $18.06 \pm 0.33$ & One peak in c & $17.92_{-0.45}^{+0.39}$ \\
c and f & $8.18 \pm 0.26$ & Main peak in c & $8.15 \pm 0.12$ \\
Chopping & frequencies & & \\
b and c & $184.2 \pm 3.1$ & No matching peak & \\
b and f & $246.1 \pm 4.1$ & No matching peak & \\
c and f & $61.9 \pm 1.0$ & One peak in c & $58.9 \pm 3.2$ \\
Orbital frequencies & & \\
b & $378.2 \pm 6.3$ & No matching peak & \\
c & $194.0 \pm 3.2$ & No matching peak & \\
$\mathrm{f}$ & $132.1 \pm 2.2$ & No matching peak & \\
\hline
\end{tabular}

Notes. Super, chopping, and orbital frequencies are given; from left to right the table shows the considered planets, the computed frequency, a match in the Ofir et al. (2018) results (if any), and the matching frequency.

found, besides the main peak at $9.82_{-0.45}^{+0.39} \times 10^{-4} \mathrm{~d}^{-1}$, another significant frequency peak at $(101.5 \pm 2.8) \times 10^{-4} \mathrm{~d}^{-1}$. The main frequency peak of Kepler-82c is at $(8.15 \pm 0.12) \times 10^{-4} \mathrm{~d}^{-1}$. In addition to that they found three more peaks in the TTVs at $((17.9,58.9,68.9) \pm 3.2) \times 10^{-4} \mathrm{~d}^{-1}$. Except for the main peak of Kepler-82b belonging to the super frequency of the near 2:1 resonance with Kepler-82c, they could not explain the detected frequencies with super frequencies of all of the mean motion resonances, orbital frequencies, chopping frequencies, or stroboscopic frequencies of the confirmed planets in the system.

In the same manner we computed the super frequencies of all mean motion resonances, orbital frequencies, and chopping frequencies of our resulting system from photodynamical analysis. The calculated frequencies are listed in Table 4. With the exception of two measured frequencies, we can explain them with interactions of the planets in the modelled system. Significantly, the super frequencies from mean motion resonances, expected to induce TTV signals, match the significant peaks found by Ofir et al. (2018). The super frequency of Kepler-82b/c corresponds to the main peak in the TTVs of Kepler-82b. Kepler-82c/f have a super frequency that explains the main peak of the TTVs in Kepler-82c. And finally, the super frequency of Kepler$82 \mathrm{~b} / \mathrm{f}$ matches a significant peak in the TTVs of Kepler-82c. 
Additionally the chopping frequency of Kepler-82c/f explains another peak of Kepler-82c TTVs. Two of the Ofir et al. (2018) frequencies with smaller confidence remain unexplained, these are the $(101.5 \pm 2.8) \times 10^{-4} \mathrm{~d}^{-1}$ frequency in planet $\mathrm{b}$ and the $(68.9 \pm 3.2) \times 10^{-4} \mathrm{~d}^{-1}$ frequency in planet $\mathrm{c}$.

For comparison, we computed the same frequencies from the 6:2:1 resonance results. In this case the super frequency of Kepler-82b/c matches, as expected, with the main peak of the Kepler-82b frequencies, and the orbital frequency of the third non-transiting planet matches the $(58.9 \pm 3.2) \times 10^{-4} \mathrm{~d}^{-1}$ peak. Besides these, no other matching frequencies were found, especially the main peak in the TTVs of Kepler-82c is not explained.

\subsection{Stellar parameters}

Transit measurements provide the information about the stellar density (Agol \& Fabrycky 2018). In our photodynamical analysis we decided to model the stellar radius while fixing the stellar mass. With this parameterisation, the density is modelled as well. We derived the stellar radius to be $R_{\mathrm{S}}=0.898_{-0.017}^{+0.038} R_{\odot}$. The high asymmetry in the uncertainties is attributed to the symmetry of the inclination of Kepler-82c around $90^{\circ}$. Together with the stellar mass from Johnson et al. (2017) of $m_{\mathrm{S}}=0.91$, this results in a stellar density of $\rho_{\mathrm{S}}=1.77_{-0.21}^{+0.12} \mathrm{~g} \mathrm{~cm}^{-3}$.

With this photodynamical-determined density and the measured stellar parameters (Petigura et al. 2017, from HIRES observations within the California-Kepler Survey) of the effective temperature $T_{\text {eff }}=5400.5 \pm 60 \mathrm{~K}$, the surface gravity $\log g=4.372 \pm 0.100$, and metallicity $\mathrm{Fe} / \mathrm{H}=0.201 \pm 0.040$ we modelled the stellar radius, mass, and age with stellar evolution models. We extracted the corresponding values from MESA (Paxton et al. 2011, 2013, 2015) evolutionary tracks interpolated by MIST (Dotter 2016; Choi et al. 2016), rejecting values of the very early evolution below 0.1 Gyr. The results are visualised in Fig. 4 as a mass-age diagram and in Fig. 5 as a radiusage diagram with the best-matching value and the $1 \sigma, 2 \sigma$, and $3 \sigma$ areas as a black star and red, orange and grey dots respectively. For comparison, the gyrochronological stellar age derived below is plotted in green; it fits within the $1 \sigma$ errorbars. The stellar parameters are derived to be $m_{\mathrm{S}}=0.94_{-0.04}^{+0.03} M_{\odot}$ for the mass, $R_{\mathrm{S}}=0.934_{-0.016}^{+0.046} R_{\odot}$ for the radius, and a stellar age of $\tau_{\text {evol }}=6.7_{-1.2}^{+3.0}$ Gyr.

We corrected the photodynamically-determined parameters that depend on stellar mass and radius, namely planetary masses, semi-major axes, and radii, with these newly determined values. The corrected values are listed in column six of Table A.2. The planetary masses and radii of Kepler-82b/c are compared in Fig. 6 with literature values of planets with masses up to $20 M_{\oplus}$ from The Extrasolar Planets Encyclopaedia ${ }^{3}$.

For testing the results of the stellar evolution model analysis we applied the gyrochronologic age determination method to the Kepler-82 system. Therefore we determined its rotation period from the Kepler long-cadence photometry excluding the transits of Kepler-82b/c (Lomb 1976; Scargle 1982; Zechmeister \& Kürster 2009). There are three small amplitude peaks in the periodogram; from these the highest-power peak corresponds to $34.7 \pm 0.8$ days. Here, the period and error are determined as the mean and standard deviation from fitting a Gaussian to the peak. We made use of Barnes (2007, 2009) gyrochronologic estimation for determining the age of Kepler-82 based on its

\footnotetext{
$\overline{3 \text { http://exoplanet.eu/ }}$
}

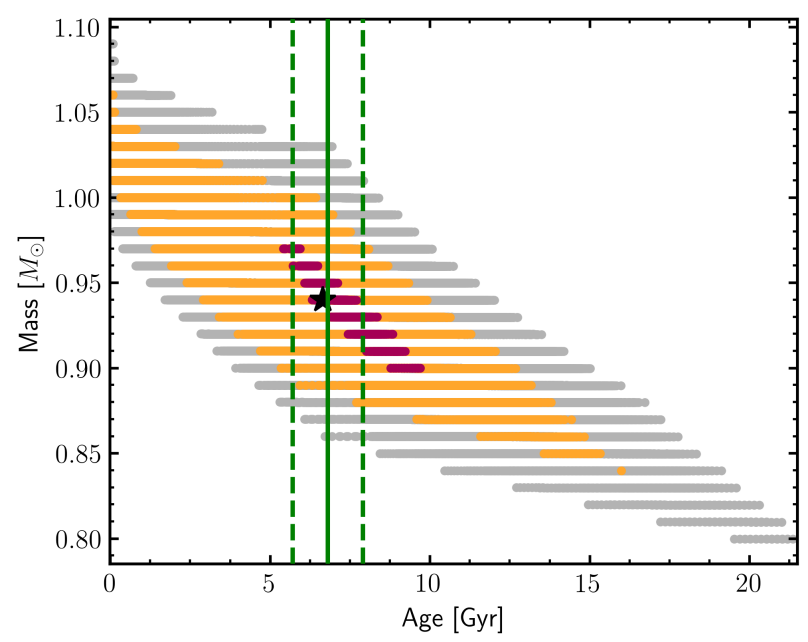

Fig. 4. Mass-age diagram of Kepler-82 from MESA stellar evolution models (MIST). The black star and the red, orange, and grey dots correspond to the best matching value and the $1 \sigma, 2 \sigma$, and $3 \sigma$ areas derived from results on the density of the whole set photodynamical modelling and from the literature values of the effective temperature, the surface gravity, and the metallicity by Petigura et al. (2017). The gyrochronologic age is indicated in green by a solid line and its $1 \sigma$ range as dashed lines.

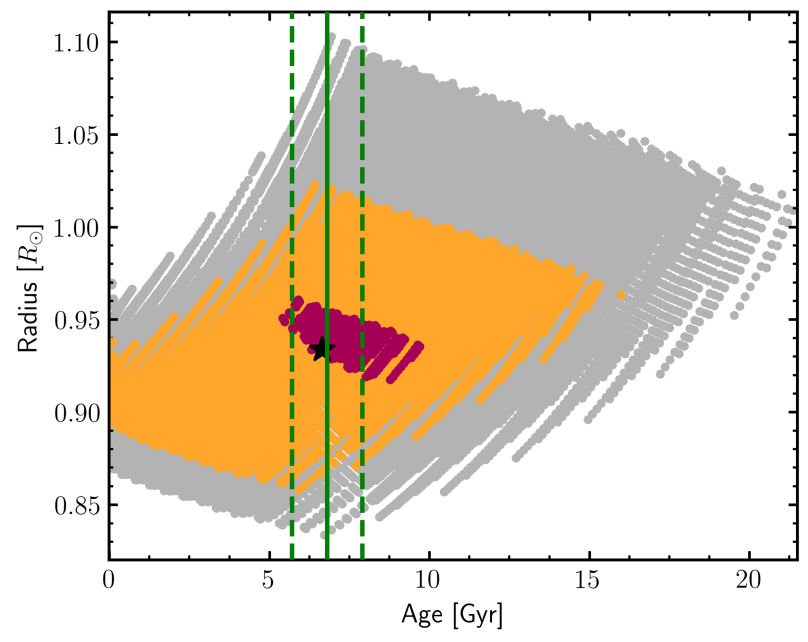

Fig. 5. Radius-age diagram of Kepler- 82 from MESA stellar evolution models (MIST). The black star and the red, orange, and grey dots correspond to the best matching value and the $1 \sigma, 2 \sigma$, and $3 \sigma$ areas derived from results on the density of the whole data set photodynamical modelling and from the literature values of the effective temperature, the surface gravity, and the metallicity by Petigura et al. (2017). The gyrochronologic age is indicated in green by a solid line and its $1 \sigma$ range as dashed lines.

rotational period:

$\log \left(\tau_{\mathrm{Gyro}}\right)=\frac{1}{n}[\log P-\log a-b \times \log (B-V-c)]$,

with $a=0.770 \pm 0.014, b=0.553 \pm 0.052, c=0.472 \pm 0.027$, and $n=0.519 \pm 0.007$. Assuming the spectral type $\mathrm{G} 7$ for Kepler-82 leads to $B-V=0.721$ (Everett et al. 2012). Following the Barnes (2009) error estimation, we derive the gyrochronological age of Kepler-82 to be $6.8 \pm 1.1 \mathrm{Gyr}$. This value fits the 


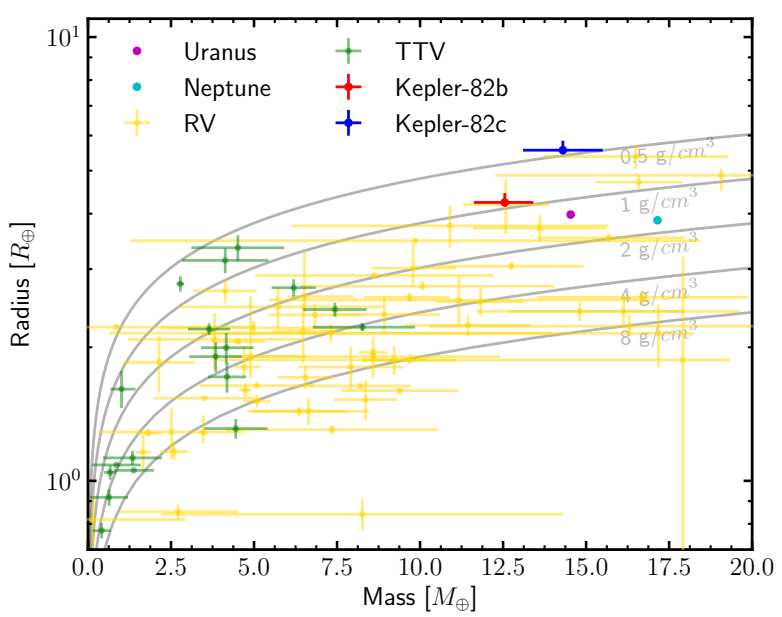

Fig. 6. Mass-radius diagram for known planets with masses up to $20 M_{\oplus}$. In yellow are the planets with mass measurements obtained by RVs and in green the planets with mass measurements obtained from TTVs. The data are given by The Extrasolar Planets Encyclopaedia. Our results are shown in red (Kepler-82b) and in blue (Kepler-82c). For comparison also the values of Uranus and Neptune are shown, the Neptune-like planet pair of our solar system.

age determined by stellar evolution models very well within the $1 \sigma$ range. It is indicated with green in the mass-age and radiusage diagram (Figs. 4 and 5) with the mean as a solid line and the standard deviation in dashed lines.

From the recently published second Gaia data release (Gaia Collaboration 2016, 2018) the effective temperature and the stellar radius were calculated to be $T_{\text {eff }}=5401 \pm 180 \mathrm{~K}$ and $R_{\mathrm{S}}=$ $0.854_{-0.046}^{+0.043} R_{\odot}$ by Berger et al. (2018). While the effective temperature perfectly fits the HIRES value, the stellar radius is significantly smaller. It fits within the $1 \sigma$ range of the value derived by the photodynamical analysis, and within the $2 \sigma$ range the value derived with the stellar evolution models. The distance of Kepler- 82 is determined to $905_{-22}^{+21}$ pc by Berger et al. (2018).

The discrepancy between stellar parameters derived by Gaia and by the combination of the photodynamical analysis and spectroscopic parameters could be a hint of another star that contaminates the light of Kepler-82. Inspecting a small region around Kepler-82 revealed a star about two magnitudes fainter at 10 arcsec distance. This distance is large enough so that the Kepler light curve should not be contaminated by this star. In the unlikely case of contamination, the radii of the planets would be underestimated by ten percent in maximum. That would make Kepler-82c to be an even more puffed-up exoplanet in the Neptune-like regime. The stellar radius and hence the density should not be affected by the light of a second star, as it is dependent upon the transit duration which is not changed. This agrees well with the fact that the photodynamical determined stellar radius matches the Gaia radius within its errorbars. It should be noted that the largest discrepancy is between the Gaia and the spectroscopic measurement, whereas the photodynamical one is in between. A further research of this deviance is beyond the scope of this paper.

\section{Conclusions}

In this work the first dynamical analysis of the Kepler-82 system was carried out, resulting in the discovery of a fifth planet.
The signal of this planet is found in the TTVs of Kepler-82c. In addition to the sinusoidal behaviour due to the interaction with Kepler-82b being near the 2:1 resonance, the TTVs show the so called chopping signal manifesting in a jump every three consecutive transits. After optimising a 2-planet photodynamical model near the 2:1 resonance to the Kepler long- and shortcadence data, we analysed the data with two different 3-planet system models. The systems differ in the ratio of the distance of the outermost fifth planet to Kepler-82c, either a $6: 2: 1$ or a 3:2:1 near-resonant system were possible. The first evidence that the 3:2:1 resonance system model was the correct assumption was provided by the $\chi_{\text {red }}^{2}$, which is a little better than the one from analysing with the 6:2:1 resonance model. The 3:2:1 resonance model is also more favourable considering the mass of planet $\mathrm{f}$ is of the same order as planets $\mathrm{b}$ and c. This system model better fits into the "peas in a pod" architecture of most systems found by Kepler (Weiss et al. 2018). This is emphasised by the light curves collected in the framework of KOINet. The three new transit observations prefer the 3:2:1 resonance model and in addition a light curve including no transit measurement was taken during the time where a transit was predicted by the 6:2:1 resonance model. Additionally, the avoidance of inclinations that lead to transits by the third planet in near 3:2 resonance fits very well with the observations. Finally, with the periods of the planets in the 3:2:1 resonance system, except for two of them the frequencies in the TTVs of Kepler-82b/c detected by Ofir et al. (2018) can be explained by the super and the chopping frequencies. The most important point here is that Ofir et al. (2018) noticed a significant offset in the highest amplitude frequency of the TTVs of Kepler-82c from the near 2:1 mean motion resonance frequency. This peak is explained by the super frequency of the near 3:2 resonance of Kepler-82f and Kepler$82 \mathrm{c}$. We conclude with announcing the detection of a fifth planet positioned in near 3:2 resonance to Kepler-82c. After the recent discovery of Kepler-411e (Sun et al. 2019), Kepler-82f is the second non-transiting planet detected via the TTVs of two other planets.

Determining the correct system architecture is important for modelling the right planet compositions. These highly depend on the assumed system architecture. The 2-planet model with significantly higher $\chi_{\text {red }}^{2}$ supposes a density ratio between planet $b$ and c of about $\sim 14$, whereas in the 3:2:1 resonance model especially the mass of Kepler-82b drops by about an order of magnitude resulting in a much more common (and reasonable) density ratio of $\sim 2$.

Acknowledgements. We acknowledge funding from the German Research Foundation (DFG) through grant DR 281/30-1. This work made use of PyAstronomy. This research has made use of the NASA Exoplanet Archive, which is operated by the California Institute of Technology, under contract with the National Aeronautics and Space Administration under the Exoplanet Exploration Program. C.v.E. acknowledges funding for the Stellar Astrophysics Centre, provided by The Danish National Research Foundation (Grant DNRF106), and support from the European Social Fund via the Lithuanian Science Council grant No. 09.3.3-LMT-K-712-01-0103. E.H. and I.R. acknowledge support from the Spanish Ministry of Economy and Competitiveness (MINECO) and the Fondo Europeo de Desarrollo Regional (FEDER) through grant ESP2016-80435-C2$1-\mathrm{R}$, as well as the support of the Generalitat de Catalunya/CERCA program. EA is supported by the United States National Science Foundation grant 1615315. Based on observations obtained with the Apache Point Observatory $3.5-\mathrm{m}$ telescope, which is owned and operated by the Astrophysical Research Consortium. We acknowledge support from the Research Council of Norway (grant 188910) to finance service observing at the NOT. SW acknowledges support for International Team 265 ("Magnetic Activity of M-type Dwarf Stars and the Influence on Habitable Extra-solar Planets") funded by the International Space Science Institute (ISSI) in Bern, Switzerland, and by the Research Council of Norway through its Centres of Excellence scheme, project number 262622 


\section{References}

Agol, E., \& Fabrycky, D. C. 2018, Transit-Timing and Duration Variations for the Discovery and Characterization of Exoplanets (Berlin: Springer), 7

Almenara, J. M., Díaz, R. F., Mardling, R., et al. 2015, MNRAS, 453, 2644

Almenara, J. M., Díaz, R. F., Bonfils, X., \& Udry, S. 2016, A\&A, 595, L5 Almenara, J. M., Díaz, R. F., Dorn, C., Bonfils, X., \& Udry, S. 2018a, MNRAS, 478, 460

Almenara, J. M., Díaz, R. F., Hébrard, G., et al. 2018b, A\&A, 615, A90

Barnes, S. A. 2007, ApJ, 669, 1167

Barnes, S. A. 2009, in The Ages of Stars, eds. E. E. Mamajek, D. R. Soderblom, \& R. F. G. Wyse, IAU Symp., 258, 345

Barros, S. C. C., Almenara, J. M., Demangeon, O., et al. 2015, MNRAS, 454, 4267

Berger, T. A., Huber, D., Gaidos, E., \& van Saders, J. L. 2018, ApJ, 866, 99

Borucki, W. J., Koch, D. G., Batalha, N., et al. 2012, ApJ, 745, 120

Bovaird, T., Lineweaver, C. H., \& Jacobsen, S. K. 2015, MNRAS, 448, 3608

Chambers, J. E. 1999, MNRAS, 304, 793

Choi, J., Dotter, A., Conroy, C., et al. 2016, ApJ, 823, 102

Claret, A., \& Bloemen, S. 2011, A\&A, 529, A75

Dotter, A. 2016, ApJS, 222, 8

Doyle, L. R., Carter, J. A., Fabrycky, D. C., et al. 2011, Science, 333, 1602

Everett, M. E., Howell, S. B., \& Kinemuchi, K. 2012, PASP, 124, 316

Fabrycky, D. C., Ford, E. B., Steffen, J. H., et al. 2012, ApJ, 750, 114

Foreman-Mackey, D., Hogg, D. W., Lang, D., \& Goodman, J. 2013, PASP, 125,306

Freudenthal, J., von Essen, C., Dreizler, S., et al. 2018, A\&A, 618, A41

Gaia Collaboration (Prusti, T., et al.) 2016, A\&A, 595, Al

Gaia Collaboration (Brown, A. G. A., et al.) 2018, A\&A, 616, A1

Goodman, J., \& Weare, J. 2010, Comm. App. Math. Comp. Sci., 5, 65
Hadden, S., \& Lithwick, Y. 2014, ApJ, 787, 80

Holczer, T., Mazeh, T., Nachmani, G., et al. 2016, ApJS, 225, 9

Johnson, J. A., Petigura, E. A., Fulton, B. J., et al. 2017, VizieR Online Data Catalog: J/AJ/154/108

Kidder, L. E. 1995, Phys. Rev. D, 52, 821

Kipping, D. M. 2010, MNRAS, 408, 1758

Kjeldsen, H., \& Frandsen, S. 1992, PASP, 104, 413

Lomb, N. R. 1976, Ap\&SS, 39, 447

Mandel, K., \& Agol, E. 2002, ApJ, 580, L171

Mazeh, T., Nachmani, G., Holczer, T., et al. 2013, ApJS, 208, 16

Morbidelli, A. 2002, Modern Celestial Mechanics: Aspects of Solar System dynamics (London: Taylor \& Francis)

Ofir, A., Xie, J.-W., Jiang, C.-F., Sari, R., \& Aharonson, O. 2018, ApJS, 234, 9

Paxton, B., Bildsten, L., Dotter, A., et al. 2011, ApJS, 192, 3

Paxton, B., Cantiello, M., Arras, P., et al. 2013, ApJS, 208, 4

Paxton, B., Marchant, P., Schwab, J., et al. 2015, ApJS, 220, 15

Petigura, E. A., Howard, A. W., Marcy, G. W., et al. 2017, AJ, 154, 107

Rowe, J. F., Bryson, S. T., Marcy, G. W., et al. 2014, ApJ, 784, 45

Scargle, J. D. 1982, ApJ, 263, 835

Southworth, J., Hinse, T. C., Jørgensen, U. G., et al. 2009, MNRAS, 396, 1023

Steele, I. A., Smith, R. J., Rees, P. C., et al. 2004, in Ground-based Telescopes, ed. J. M. Oschmann, Jr., Proc. SPIE, 5489, 679

Steffen, J. H., Fabrycky, D. C., Agol, E., et al. 2013, MNRAS, 428, 1077

Sun, L., Ioannidis, P., Gu, S., et al. 2019, A\&A, 624, A15

von Essen, C., Schröter, S., Agol, E., \& Schmitt, J. H. M. M. 2013, A\&A, 555, A92

von Essen, C., Ofir, A., Dreizler, S., et al. 2018, A\&A, 615, A79

Weiss, L. M., Marcy, G. W., Petigura, E. A., et al. 2018, AJ, 155, 48

Xie, J.-W. 2013, ApJS, 208, 22

Zechmeister, M., \& Kürster, M. 2009, A\&A, 496, 577 
Appendix A: Additional plots and tables

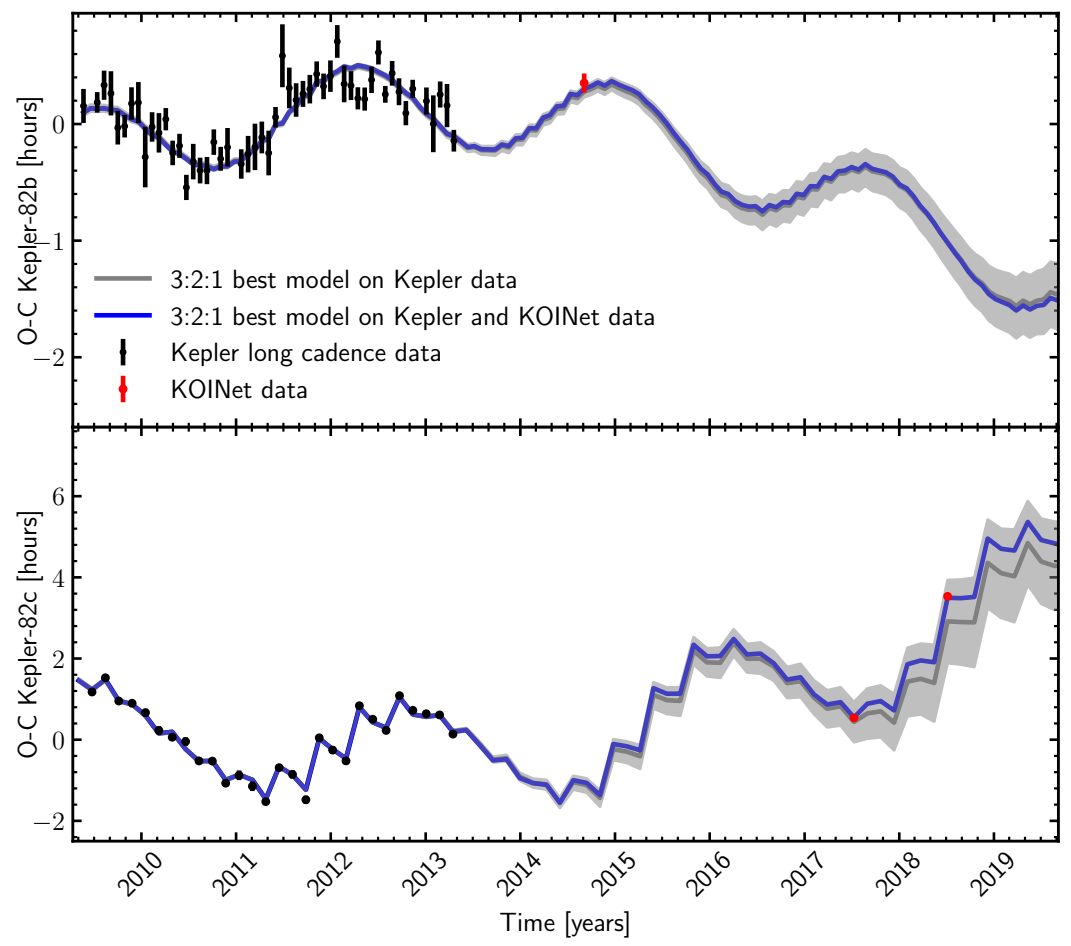

Fig. A.1. O-C diagram of Kepler-82b at the top and Kepler-82c at the bottom with transit times from modelling the transits individually. The black points refer to the transit data from the Kepler telescope. The red points are the individual transit times from the new KOINet observations. The grey area indicates the $68.3 \%$ confidence interval of the $3: 2: 1$ resonance model fitted to the Kepler long- and short-cadence data only, the grey line is the best $\chi^{2}$ solution. The blue area indicates the same model solution applied to Kepler and KOINet data respectively.

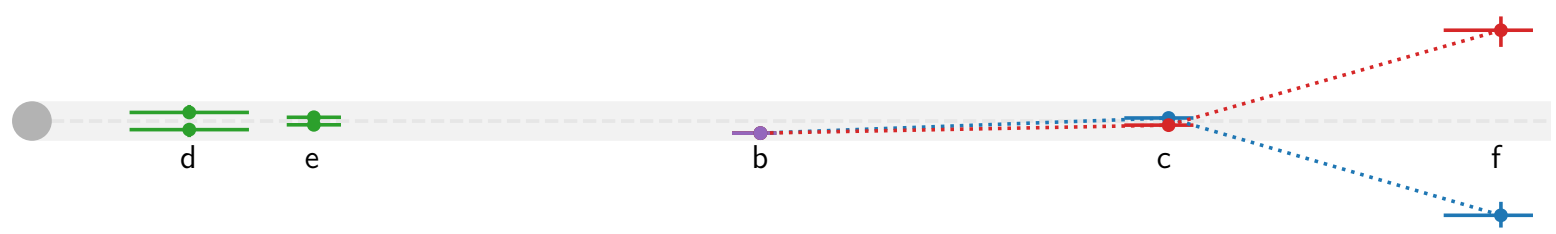

Fig. A.2. Configurations of the Kepler- 82 system. With the star in grey on the left side and the observer on the right side this shows the two different configurations: $\mathrm{b}$ in violet has the same position in both, $\mathrm{c}$ and $\mathrm{f}$ in red shows configuration I and in blue configuration II. The grey area indicates the region of impact parameters below one. The distances are true to scale with the stellar radius. Additionally, the two inner planets are plotted in green, the data are taken from the NASA Exoplanet Archive. They are plotted on both sides because we did not include them in the photodynamical analysis and hence we do not know how they behave in the two configurations. 


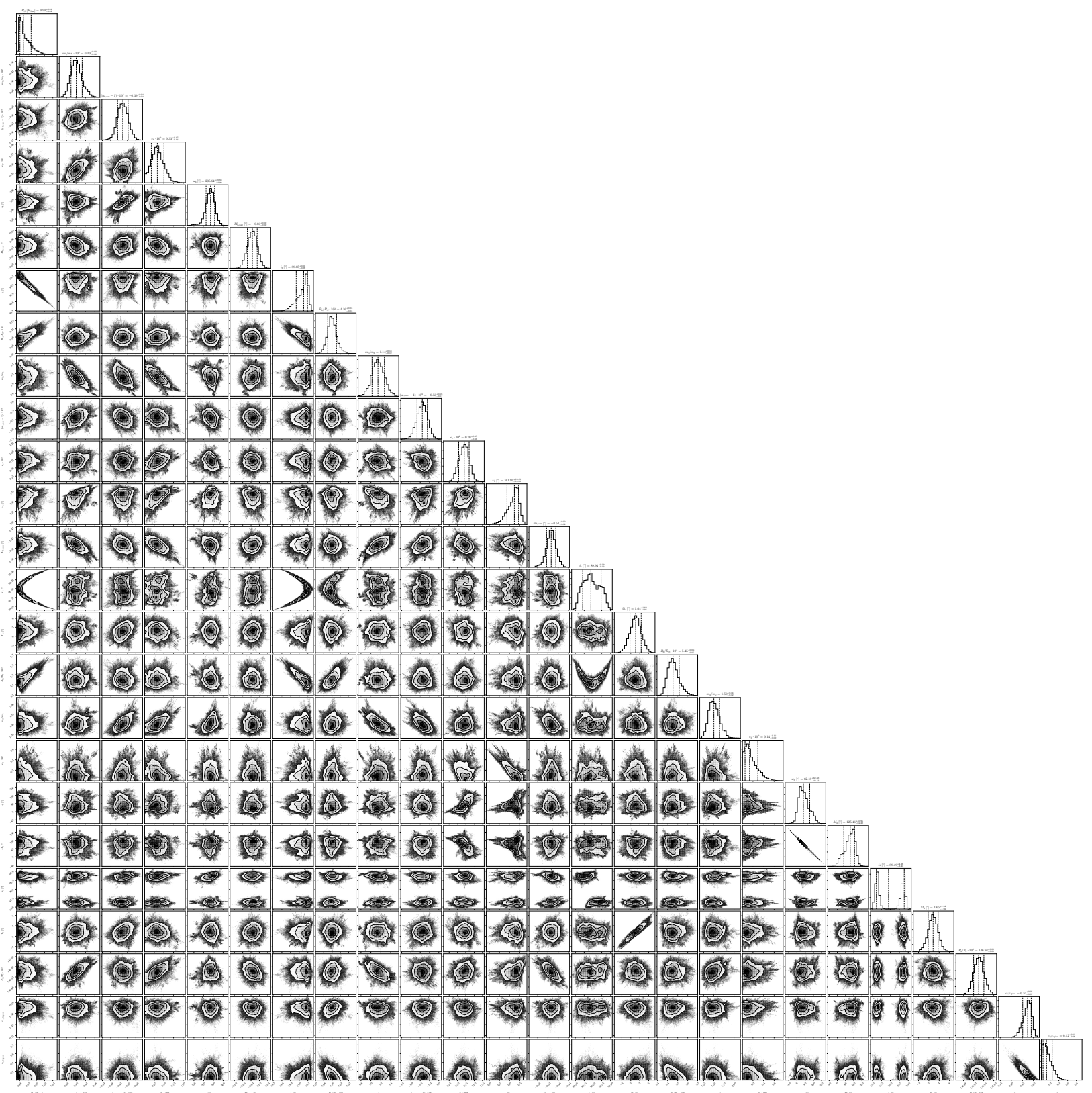

Fig. A.3. Correlation plot of all fit parameters from modelling the 3:2:1 resonance model to Kepler long- and short-cadence and KOINet data. 
A\&A 628, A108 (2019)

Table A.1. Ephemerides E and transit time predictions in BJD-2 400000.0 from modelling Kepler and KOINet data for the next 15 yr.

\begin{tabular}{|c|c|c|c|c|c|c|c|}
\hline$E$ & BJD & $E$ & BJD & $E$ & BJD & E & BJD \\
\hline \multicolumn{8}{|c|}{ Kepler-82b } \\
\hline 133 & $58491.2327(78)$ & 188 & $59945.6559(140)$ & 243 & $61400.0816(164)$ & 298 & $62854.5228(154)$ \\
\hline 134 & $58517.6757(80)$ & 189 & $59972.1003(141)$ & 244 & $61426.5237(164)$ & 299 & $62880.9705(152)$ \\
\hline 135 & $58544.1187(81)$ & 190 & $59998.5465(141)$ & 245 & $61452.9658(165)$ & 300 & $62907.4159(152)$ \\
\hline 136 & $58570.5609(83)$ & 191 & $60024.9908(141)$ & 246 & $61479.4095(165)$ & 301 & $62933.8627(151)$ \\
\hline 137 & $58597.0068(84)$ & 192 & $60051.4355(142)$ & 247 & $61505.8527(166)$ & 302 & $62960.3066(151)$ \\
\hline 138 & $58623.4495(86)$ & 193 & $60077.8793(143)$ & 248 & $61532.2962(166)$ & 303 & $62986.7544(150)$ \\
\hline 139 & $58649.8947(86)$ & 194 & $60104.3241(142)$ & 249 & $61558.7399(167)$ & 304 & $63013.1981(150)$ \\
\hline 140 & $58676.3391(88)$ & 195 & $60130.7667(143)$ & 250 & $61585.1852(167)$ & 305 & $63039.6433(149)$ \\
\hline 141 & $58702.7857(88)$ & 196 & $60157.2097(144)$ & 251 & $61611.6291(167)$ & 306 & $63066.0878(149)$ \\
\hline 142 & $58729.2287(90)$ & 197 & $60183.6534(145)$ & 252 & $61638.0751(167)$ & 307 & $63092.5323(148)$ \\
\hline 143 & $58755.6765(90)$ & 198 & $60210.0953(145)$ & 253 & $61664.5197(168)$ & 308 & $63118.9747(148)$ \\
\hline 144 & $58782.1205(92)$ & 199 & $60236.5371(147)$ & 254 & $61690.9672(167)$ & 309 & $63145.4188(148)$ \\
\hline 145 & $58808.5676(91)$ & 200 & $60262.9801(148)$ & 255 & $61717.4115(168)$ & 310 & $63171.8614(149)$ \\
\hline 146 & $58835.0118(93)$ & 201 & $60289.4217(148)$ & 256 & $61743.8595(167)$ & 311 & 63 198.3035(149) \\
\hline 147 & $58861.4591(93)$ & 202 & $60315.8630(150)$ & 257 & $61770.3053(167)$ & 312 & $63224.7456(149)$ \\
\hline 148 & $58887.9031(94)$ & 203 & $60342.3056(151)$ & 258 & $61796.7530(166)$ & 313 & $63251.1875(150)$ \\
\hline 149 & $58914.3495(94)$ & 204 & $60368.7475(152)$ & 259 & $61823.1979(166)$ & 314 & $63277.6289(150)$ \\
\hline 150 & 58 940.7934(96) & 205 & $60395.1890(153)$ & 260 & $61849.6473(164)$ & 315 & $63304.0698(151)$ \\
\hline 151 & $58967.2398(95)$ & 206 & $60421.6317(155)$ & 261 & $61876.0919(165)$ & 316 & $63330.5110(152)$ \\
\hline 152 & $58993.6827(97)$ & 207 & $60448.0743(156)$ & 262 & $61902.5393(163)$ & 317 & $63356.9527(152)$ \\
\hline 153 & $59020.1276(97)$ & 208 & $60474.5168(157)$ & 263 & $61928.9854(163)$ & 318 & $63383.3930(154)$ \\
\hline 154 & $59046.5716(98)$ & 209 & $60500.9603(158)$ & 264 & $61955.4328(161)$ & 319 & $63409.8340(155)$ \\
\hline 155 & $59073.0148(99)$ & 210 & $60527.4037(159)$ & 265 & $61981.8771(161)$ & 320 & $63436.2758(155)$ \\
\hline 156 & $59099.4571(100)$ & 211 & $60553.8485(160)$ & 266 & $62008.3245(160)$ & 321 & $63462.7167(156)$ \\
\hline 157 & $59125.9010(101)$ & 212 & $60580.2918(162)$ & 267 & $62034.7689(159)$ & 322 & $63489.1580(158)$ \\
\hline 158 & $59152.3426(102)$ & 213 & $60606.7375(162)$ & 268 & $62061.2140(158)$ & 323 & $63515.6009(159)$ \\
\hline 159 & $59178.7840(104)$ & 214 & $60633.1824(163)$ & 269 & $62087.6584(158)$ & 324 & $63542.0429(160)$ \\
\hline 160 & $59205.2266(105)$ & 215 & $60659.6285(163)$ & 270 & 62 114.1031(157) & 325 & $63568.4846(161)$ \\
\hline 161 & $59231.6677(107)$ & 216 & $60686.0730(165)$ & 271 & 62140.5 & 326 & $4.9293(161)$ \\
\hline 162 & $59258.1085(108)$ & 217 & $60712.5216(164)$ & 272 & $62166.9898(156)$ & 327 & $63621.3717(162)$ \\
\hline 163 & $59284.5502(110)$ & 218 & $60738.9659(165)$ & 273 & $62193.4328(155)$ & 328 & $63647.8158(163)$ \\
\hline 164 & $59310.9914(112)$ & 219 & $60765.4137(165)$ & 274 & $62219.8757(154)$ & 329 & $63674.2594(164)$ \\
\hline 165 & $59337.4321(114)$ & 220 & $60791.8599(166)$ & 275 & $62246.3176(154)$ & 330 & $63700.7052(164)$ \\
\hline 166 & $59363.8735(116)$ & 221 & $60818.3081(164)$ & 276 & $62272.7598(155)$ & 331 & $63727.1480(165)$ \\
\hline 167 & $59390.3151(117)$ & 222 & $60844.7527(166)$ & 277 & $62299.2023(154)$ & 332 & $63753.5951(165)$ \\
\hline 168 & $59416.7567(119)$ & 223 & $60871.2021(165)$ & 278 & $62325.6436(154)$ & 333 & $63780.0389(166)$ \\
\hline 169 & $59443.1983(121)$ & 224 & $60897.6472(165)$ & 279 & $62352.0850(154)$ & 334 & 63 806.4861(165) \\
\hline 170 & $59469.6408(123)$ & 225 & $60924.0946(164)$ & 280 & $62378.5279(154)$ & 335 & $63832.9296(167)$ \\
\hline 171 & $59496.0836(125)$ & 226 & $60950.5402(165)$ & 281 & $62404.9690(154)$ & 336 & $63859.3772(166)$ \\
\hline 172 & $59522.5265(126)$ & 227 & $60976.9875(163)$ & 282 & $62431.4105(155)$ & 337 & $63885.8215(167)$ \\
\hline 173 & $59548.9693(128)$ & 228 & $61003.4318(163)$ & 283 & $62457.8536(155)$ & 338 & $63912.2682(166)$ \\
\hline 174 & $59575.4148(129)$ & 229 & $61029.8781(163)$ & 284 & $62484.2956(155)$ & 339 & $63938.7120(167)$ \\
\hline 175 & $59601.8574(131)$ & 230 & $61056.3226(163)$ & 285 & $62510.7379(155)$ & 340 & $63965.1594(167)$ \\
\hline 176 & $59628.3030(132)$ & 231 & $61082.7678(162)$ & 286 & $62537.1817(156)$ & 341 & $63991.6023(168)$ \\
\hline 177 & $59654.7478(134)$ & 232 & 61 109.2114(162) & 287 & $62563.6254(156)$ & 342 & $64018.0479(167)$ \\
\hline 178 & $59681.1942(134)$ & 233 & $61135.6556(162)$ & 288 & $62590.0680(156)$ & 343 & $64044.4922(168)$ \\
\hline 179 & $59707.6379(136)$ & 234 & 61 162.0995(161) & 289 & 62 616.5136(156) & 344 & $64070.9364(168)$ \\
\hline 180 & $59734.0866(136)$ & 235 & 61 188.5422(162) & 290 & $62642.9573(156)$ & 345 & $64097.3789(169)$ \\
\hline 181 & $59760.5308(137)$ & 236 & $61214.9851(162)$ & 291 & $62669.4031(155)$ & 346 & $64123.8236(170)$ \\
\hline 182 & $59786.9783(137)$ & 237 & $61241.4285(162)$ & 292 & $62695.8471(156)$ & 347 & $64150.2657(171)$ \\
\hline 183 & $59813.4238(139)$ & 238 & $61267.8702(162)$ & 293 & $62722.2942(155)$ & 348 & $64176.7075(172)$ \\
\hline 184 & $59839.8718(138)$ & 239 & $61294.3120(162)$ & 294 & $62748.7387(155)$ & 349 & $64203.1501(173)$ \\
\hline 185 & $59866.3161(140)$ & 240 & $61320.7550(162)$ & 295 & $62775.1860(155)$ & 350 & $64229.5917(174)$ \\
\hline 186 & $59892.7641(139)$ & 241 & $61347.1967(163)$ & 296 & $62801.6304(155)$ & 351 & $64256.0326(175)$ \\
\hline 187 & $59919.2089(141)$ & 242 & $61373.6382(163)$ & 297 & $62828.0789(153)$ & 352 & $64282.4739(177)$ \\
\hline
\end{tabular}

Notes. The median and standard deviation solution of 1000 randomly chosen good models. Reference times for ephemeris $E=1$ : $T_{\mathrm{b}}=54974.2409(12)$ and $T_{\mathrm{c}}=54955.5862(22)$. 
J. Freudenthal et al.: Kepler Object of Interest Network. III.

Table A.1. continued.

\begin{tabular}{|c|c|c|c|c|c|c|c|}
\hline$E$ & BJD & $E$ & BJD & $E$ & BJD & $E$ & BJD \\
\hline \multicolumn{8}{|c|}{ Kepler-82c } \\
\hline 69 & $58511.9213(51)$ & 97 & $59955.0537(94)$ & 125 & $61398.0596(170)$ & 153 & $62841.1069(181)$ \\
\hline 70 & $58563.4587(50)$ & 98 & $60006.5884(106)$ & 126 & $61449.5997(168)$ & 154 & $62892.6426(169)$ \\
\hline 71 & $58615.0271(63)$ & 99 & $60058.1120(99)$ & 127 & $61501.1194(171)$ & 155 & $62944.1653(168)$ \\
\hline 72 & $58666.5477(58)$ & 100 & $60109.6316(109)$ & 128 & $61552.6564(211)$ & 156 & $62995.7474(207)$ \\
\hline 73 & $58718.0834(51)$ & 101 & $60161.1717(114)$ & 129 & $61604.1925(205)$ & 157 & $63047.2834(187)$ \\
\hline 74 & $58769.6185(58)$ & 102 & $60212.6931(118)$ & 130 & $61655.7116(210)$ & 158 & $63098.8153(183)$ \\
\hline 75 & $58821.1380(52)$ & 103 & $60264.2227(140)$ & 131 & $61707.2758(266)$ & 159 & $63150.4148(180)$ \\
\hline 76 & $58872.6773(49)$ & 104 & $60315.7634(137)$ & 132 & $61758.8083(257)$ & 160 & 63 201.9487(157) \\
\hline 77 & $58924.1995(49)$ & 105 & $60367.2860(141)$ & 133 & $61810.3307(263)$ & 161 & $63253.4866(151)$ \\
\hline 78 & $58975.7233(48)$ & 106 & $60418.8402(184)$ & 134 & $61861.9183(295)$ & 162 & $63305.0819(114)$ \\
\hline 79 & $59027.2653(47)$ & 107 & $60470.3785(177)$ & 135 & $61913.4470(279)$ & 163 & $63356.6105(113)$ \\
\hline 80 & $59078.7886(49)$ & 108 & $60521.9032(183)$ & 136 & $61964.9745(281)$ & 164 & 63 408.1497(119) \\
\hline 81 & $59130.3301(62)$ & 109 & $60573.4843(225)$ & 137 & $62016.5681(261)$ & 165 & $63459.7170(151)$ \\
\hline 82 & $59181.8732(58)$ & 110 & $60625.0176(219)$ & 138 & $62068.0914(244)$ & 166 & $63511.2413(161)$ \\
\hline 83 & $59233.4008(59)$ & 111 & $60676.5439(228)$ & 139 & $62119.6225(243)$ & 167 & $63562.7815(162)$ \\
\hline 84 & $59284.9733(87)$ & 112 & $60728.1367(240)$ & 140 & $62171.2001(178)$ & 168 & $63614.3177(210)$ \\
\hline 85 & $59336.5156(80)$ & 113 & $60779.6613(234)$ & 141 & $62222.7205(171)$ & 169 & 63 665.8397(210) \\
\hline 86 & $59388.0482(83)$ & 114 & $60831.1886(243)$ & 142 & $62274.2547(175)$ & 170 & $63717.3808(207)$ \\
\hline 87 & $59439.6448(102)$ & 115 & $60882.7736(213)$ & 143 & $62325.8041(127)$ & 171 & $63768.9029(219)$ \\
\hline 88 & $59491.1819(98)$ & 116 & $60934.2908(211)$ & 144 & $62377.3249(129)$ & 172 & $63820.4267(202)$ \\
\hline 89 & $59542.7160(107)$ & 117 & $60985.8186(220)$ & 145 & $62428.8641(135)$ & 173 & $63871.9669(202)$ \\
\hline 90 & $59594.3141(102)$ & 118 & $61037.3776(167)$ & 146 & $62480.3915(129)$ & 174 & 63 923.4873(200) \\
\hline 91 & $59645.8400(104)$ & 119 & $61088.8929(171)$ & 147 & $62531.9134(133)$ & 175 & $63975.0305(161)$ \\
\hline 92 & $59697.3710(116)$ & 120 & $61140.4255(182)$ & 148 & $62583.4535(135)$ & 176 & $64026.5707(159)$ \\
\hline 93 & $59748.9480(90)$ & 121 & $61191.9578(149)$ & 149 & $62634.9733(137)$ & 177 & $64078.0964(152)$ \\
\hline 94 & $59800.4643(96)$ & 122 & $61243.4763(155)$ & 150 & $62686.5010(148)$ & 178 & $64129.6718(140)$ \\
\hline 95 & $59851.9942(111)$ & 123 & $61295.0151(161)$ & 151 & $62738.0382(144)$ & 179 & 64 181.2144(132) \\
\hline 96 & $59903.5382(85)$ & 124 & $61346.5365(157)$ & 152 & $62789.5566(144)$ & 180 & $64232.7492(127)$ \\
\hline
\end{tabular}


A\&A 628, A108 (2019)

Table A.2. Stellar and planetary parameters from the photodynamical modelling of the 2:1 resonance solution on Kepler long- and short-cadence data, the 6:2:1 resonance solution on Kepler data, the 3:2:1 resonance solution on Kepler data, the 3:2:1 resonance solution on Kepler data and the three KOINet transit light curves, and some corrections from investigating stellar evolution models in Sect. 5.4.

\begin{tabular}{|c|c|c|c|c|c|}
\hline Parameter & $\begin{array}{c}\text { Kepler data } \\
2: 1\end{array}$ & $\begin{array}{c}\text { Kepler data } \\
6: 2: 1\end{array}$ & $\begin{array}{c}\text { Kepler data } \\
3: 2: 1\end{array}$ & $\begin{array}{c}\text { Kepler \& } \\
\text { KOINet data } \\
3: 2: 1\end{array}$ & $\begin{array}{l}\text { MESA } \\
3: 2: 1 \\
\end{array}$ \\
\hline \multicolumn{6}{|l|}{ Kepler-82b } \\
\hline$m_{\mathrm{b}} / m_{\mathrm{S}}$ & $0.000682_{-0.000022}^{+0.000021}$ & $0.0000563_{-0.0000076}^{+0.0000072}$ & $0.0000374_{-0.0000055}^{+0.0000069}$ & $0.0000401_{-0.0000025}^{+0.000028}$ & \\
\hline$m_{\mathrm{b}}^{*}\left(M_{\oplus}\right)$ & $207 \pm 10$ & $17.1_{-2.4}^{+2.3}$ & $11.3_{-1.7}^{+2.1}$ & $12.15_{-0.87}^{+0.96}$ & $12.5_{-1.0}^{+0.9}$ \\
\hline$a_{\mathrm{b}, \mathrm{corr}}$ & $0.9999097_{-0.0000066}^{+0.0000067}$ & $0.999927 \pm 0.000011$ & $0.9999605_{-0.0000058}^{+0.0000057}$ & $0.9999606_{-0.0000041}^{+0.0000042}$ & \\
\hline$a_{\mathrm{b}}^{*}(\mathrm{AU})$ & $0.1684 \pm 0.0021$ & $0.1683 \pm 0.0020$ & $0.1683 \pm 0.0020$ & $0.1683 \pm 0.0020$ & $0.1702_{-0.0024}^{+0.0018}$ \\
\hline$P_{\mathrm{b}}^{*}(\mathrm{~d})$ & $26.44 \pm 0.48$ & $26.44 \pm 0.48$ & $26.44 \pm 0.48$ & $26.44 \pm 0.48$ & $26.44_{-0.56}^{+0.42}$ \\
\hline$e_{b}$ & $0.01737_{-0.00067}^{+0.00068}$ & $0.0024_{-0.0013}^{+0.0011}$ & $0.0030_{-0.0019}^{+0.0024}$ & $0.0033_{-0.0017}^{+0.0019}$ & \\
\hline$i_{\mathrm{b}}\left({ }^{\circ}\right)$ & $88.49_{-0.16}^{+0.18}$ & $89.109_{-0.066}^{+0.032}$ & $89.031_{-0.096}^{+0.068}$ & $89.052_{-0.096}^{+0.049}$ & \\
\hline$\Omega_{\mathrm{b}}\left(^{\circ}\right)$ & \multicolumn{4}{|c|}{0 (fixed) } & \\
\hline$\omega_{\mathrm{b}}\left({ }^{\circ}\right)$ & $254.6 \pm 1.3$ & $214_{-43}^{+37}$ & $231_{-46}^{+29}$ & $236_{-26}^{+23}$ & \\
\hline$M_{\mathrm{b}, \text { corr }}\left({ }^{\circ}\right)$ & $0.079 \pm 0.018$ & $-0.094_{-0.021}^{+0.023}$ & $-0.026_{-0.021}^{+0.019}$ & $-0.025_{-0.020}^{+0.019}$ & \\
\hline$M_{\mathrm{b}}^{*}\left({ }^{\circ}\right)$ & $354.6 \pm 1.3$ & $35_{-37}^{+43}$ & $18_{-29}^{+46}$ & $13_{-23}^{+26}$ & \\
\hline$R_{\mathrm{b}} / R_{\mathrm{S}}$ & $0.04428_{-0.00135}^{+0.00127}$ & $0.04125_{-0.00039}^{+0.00040}$ & $0.04166_{-0.00050}^{+0.00053}$ & $0.04159_{-0.00045}^{+0.00049}$ & \\
\hline$R_{\mathrm{b}}^{*}\left(R_{\oplus}\right)$ & $5.74_{-0.57}^{+0.54}$ & $3.96_{-0.06}^{+0.15}$ & $4.13_{-0.16}^{+0.24}$ & $4.07_{-0.10}^{+0.24}$ & $4.24_{-0.09}^{+0.22}$ \\
\hline$\rho_{\mathrm{b}}^{*}\left(\mathrm{~g} \mathrm{~cm}^{-3}\right)$ & $6.0_{-1.4}^{+2.2}$ & $\begin{array}{r}1.49_{-0.21}^{+0.19} \\
\end{array}$ & $0.88_{-0.17}^{+0.19}$ & $0.98_{-0.16}^{+0.11}$ & \\
\hline \multicolumn{6}{|l|}{ Kepler-82c } \\
\hline$m_{\mathrm{c}} / m_{\mathrm{b}}$ & $0.156 \pm 0.012$ & $1.06 \pm 0.14$ & $1.23_{-0.20}^{+0.21}$ & $1.14_{-0.13}^{+0.14}$ & \\
\hline$m_{\mathrm{c}}^{*}\left(M_{\oplus}\right)$ & $32.2 \pm 2.2$ & $18.0_{-2.0}^{+1.8}$ & $13.9_{-1.6}^{+1.4}$ & $13.9_{-1.2}^{+1.3}$ & $14.3 \pm 1.3$ \\
\hline$a_{\mathrm{c}, \mathrm{corr}}$ & $1.003856_{-0.000099}^{+0.000095}$ & $0.999860_{-0.000044}^{+0.000041}$ & $0.999934_{-0.000029}^{+0.0000030}$ & $0.999947_{-0.000019}^{+0.000018}$ & \\
\hline$a_{\mathrm{c}}^{*}(\mathrm{AU})$ & $0.2637 \pm 0.0032$ & $0.2626 \pm 0.0032$ & $0.2626 \pm 0.0032$ & $0.2626 \pm 0.0032$ & $0.2655_{-0.0038}^{+0.0028}$ \\
\hline$P_{\mathrm{c}}^{*}(\mathrm{~d})$ & $51.84 \pm 0.95$ & $51.53 \pm 0.94$ & $51.53 \pm 0.94$ & $51.54 \pm 0.94$ & $51.5_{-1.1}^{+0.8}$ \\
\hline$e_{\mathrm{c}}$ & $0.0685_{-0.0014}^{+0.0015}$ & $0.0103_{-0.0034}^{+0.0030}$ & $0.0072_{-0.0017}^{+0.0019}$ & $0.0070_{-0.0018}^{+0.0016}$ & \\
\hline$i_{\mathrm{c}}\left(^{\circ}\right)$ config. I & $89.19_{-0.10}^{+0.13}$ & $89.96 \pm 0.18$ & $90.18_{-0.18}^{+0.17}$ & $90.15_{-0.22}^{+0.18}$ & \\
\hline$i_{\mathrm{c}}\left(^{\circ}\right)$ config. II & $90.76_{-0.13}^{+0.12}$ & - & $89.73_{-0.12}^{+0.14}$ & $89.78_{-0.15}^{+0.17}$ & \\
\hline$\Omega_{\mathrm{c}}\left({ }^{\circ}\right)$ config. I & $0.01_{-0.10}^{+0.09}$ & $1.6_{-1.4}^{+1.3}$ & $1.8_{-2.1}^{+2.0}$ & $1.6 \pm 2.1$ & \\
\hline$\Omega_{\mathrm{c}}\left({ }^{\circ}\right)$ config. II & $0.29 \pm 0.10$ & - & - & - & \\
\hline$\omega_{\mathrm{c}}\left({ }^{\circ}\right)$ & $271.50_{-0.67}^{+0.69}$ & $268_{-16}^{+13}$ & $161_{-24}^{+12}$ & $162_{-20}^{+12}$ & \\
\hline$M_{\mathrm{c}, \mathrm{corr}}\left({ }^{\circ}\right)$ & $-0.952_{-0.044}^{+0.042}$ & $-0.554_{-0.019}^{+0.018}$ & $-0.509_{-0.019}^{+0.020}$ & $-0.507 \pm 0.020$ & \\
\hline$M_{\mathrm{c}}^{*}\left({ }^{\circ}\right)$ & $19.99_{-0.78}^{+0.75}$ & $25_{-13}^{+16}$ & $132_{-12}^{+23}$ & $131_{-12}^{+20}$ & \\
\hline$R_{\mathrm{c}} / R_{\mathrm{S}}$ & $0.0578_{-0.0019}^{+0.0017}$ & $0.05423_{-0.00047}^{+0.00048}$ & $0.05461_{-0.00053}^{+0.00073}$ & $0.05453_{-0.00053}^{+0.00068}$ & \\
\hline$R_{\mathrm{c}}^{*}\left(R_{\oplus}\right)$ & $7.49_{-0.77}^{+0.72}$ & $5.19_{-0.06}^{+0.20}$ & $5.41_{-0.20}^{+0.33}$ & $5.34_{-0.13}^{+0.32}$ & $5.56_{-0.11}^{+0.28}$ \\
\hline$\rho_{\mathrm{c}}^{*}\left(\mathrm{~g} \mathrm{~cm}^{-3}\right)$ & $0.42_{-0.11}^{+0.16}$ & $0.693_{-0.083}^{+0.077}$ & $0.480_{-0.088}^{+0.085}$ & $0.494_{-0.083}^{+0.070}$ & \\
\hline \multicolumn{6}{|l|}{ Kepler-82f } \\
\hline$m_{\mathrm{f}} / m_{\mathrm{c}}$ & - & $23.0_{-2.1}^{+2.8}$ & $1.50_{-0.16}^{+0.17}$ & $1.50_{-0.13}^{+0.16}$ & \\
\hline$m_{\mathrm{f}}^{*}\left(M_{\oplus}\right)$ & - & $415 \pm 23$ & $20.9 \pm 1.0$ & $20.9 \pm 1.0$ & $21.6_{-1.2}^{+1.0}$ \\
\hline$P_{\mathrm{f}} / P_{\mathrm{c}}$ & - & $3.1245_{-0.0027}^{+0.0023}$ & $1.46969_{-0.00041}^{+0.00044}$ & $1.46940_{-0.00022}^{+0.00023}$ & \\
\hline$P_{\mathrm{f}}^{*}(\mathrm{~d})$ & - & $161.03_{-0.14}^{+0.12}$ & $75.747_{-0.021}^{+0.023}$ & $75.732 \pm 0.012$ & \\
\hline$a_{\mathrm{f}}^{*}(\mathrm{AU})$ & - & $0.5616 \pm 0.0068$ & $0.3395 \pm 0.0041$ & $0.3395 \pm 0.0041$ & $0.3432_{-0.0049}^{+0.0037}$ \\
\hline$e_{\mathrm{f}}$ & - & $0.0912_{-0.0047}^{+0.0066}$ & $0.0016_{-0.0013}^{+0.0022}$ & $0.0014_{-0.0010}^{+0.0018}$ & \\
\hline$i_{\mathrm{f}}\left({ }^{\circ}\right)$ config. I & - & $90.6_{-2.9}^{+3.7}$ & $86.21_{-0.68}^{+0.78}$ & $86.30 \pm 0.56$ & \\
\hline
\end{tabular}

Notes. Listed are the median values and $68.26 \%$ confidence interval from the MCMC posterior distribution. The osculating orbital elements are given at a reference time, $\mathrm{BJD}=2454$ 933.0. ${ }^{(*)}$ Derived, not fitted parameters. 
J. Freudenthal et al.: Kepler Object of Interest Network. III.

Table A.2. continued.

\begin{tabular}{|c|c|c|c|c|c|}
\hline Parameter & Kepler data & Kepler data & Kepler data & $\begin{array}{l}\text { Kepler \& } \\
\text { KOINet data }\end{array}$ & MESA \\
\hline & $2: 1$ & $6: 2: 1$ & $3: 2: 1$ & $3: 2: 1$ & $3: 2: 1$ \\
\hline$i_{\mathrm{f}}\left({ }^{\circ}\right)$ config. II & - & - & $93.63_{-0.67}^{+0.61}$ & $93.62_{-0.72}^{+0.56}$ & \\
\hline$\Omega_{\mathrm{f}}\left({ }^{\circ}\right)$ & - & $1.7_{-1.7}^{+1.2}$ & $1.9 \pm 2.2$ & $1.6_{-2.1}^{+2.2}$ & \\
\hline$\omega_{\mathrm{f}}\left({ }^{\circ}\right)$ & - & $279.5_{-4.7}^{+4.3}$ & $77_{-62}^{+67}$ & $62_{-47}^{+70}$ & \\
\hline$M_{\mathrm{f}}\left({ }^{\circ}\right)$ & - & $92.5_{-4.4}^{+4.5}$ & $111_{-67}^{+61}$ & $125_{-70}^{+47}$ & \\
\hline \multicolumn{6}{|l|}{ Kepler-82 } \\
\hline$m_{\mathrm{S}}\left(M_{\odot}\right)$ & \multicolumn{4}{|c|}{$0.91 \pm 0.03$ (fixed, Johnson et al. 2017) } & $0.94_{-0.04}^{+0.03}$ \\
\hline$R_{\mathrm{S}}\left(R_{\odot}\right)$ & $1.186_{-0.085}^{+0.082}$ & $0.880_{-0.010}^{+0.025}$ & $0.907_{-0.026}^{+0.042}$ & $0.898_{-0.018}^{+0.042}$ & $0.934_{-0.016}^{+0.046}$ \\
\hline$\rho_{\mathrm{S}}^{*}\left(\mathrm{~g} \mathrm{~cm}^{-3}\right)$ & $0.77_{-0.14}^{+0.19}$ & $1.89_{-0.15}^{+0.06}$ & $1.72_{-0.22}^{+0.16}$ & $1.77_{-0.23}^{+0.11}$ & \\
\hline$c_{1, \text { Kepler }}$ & $0.31_{-0.19}^{+0.23}$ & $0.498_{-0.074}^{+0.064}$ & $0.515_{-0.062}^{+0.048}$ & $0.522_{-0.075}^{+0.054}$ & \\
\hline$c_{2, \text { Kepler }}$ & $0.66_{-0.36}^{+0.26}$ & $0.19_{-0.11}^{+0.13}$ & $0.134_{-0.073}^{+0.090}$ & $0.12_{-0.09}^{+0.14}$ & \\
\hline
\end{tabular}




\section{Chapter 4}

\section{Summary, conclusion and outlook}

In this thesis, ground-based follow-up observations of two Kepler systems were evaluated and a photodynamical model was developed for a comprehensive and selfconsistent analysis of the systems. The results of the photodynamical analysis of Kepler and KOINet data for the Kepler-9b/c and the Kepler-82b/c system are summarised and discussed in the following sections. Afterwards, both systems are examined in context of the topical literature given in the introduction (chapter 1), especially on multi-planet systems. Furthermore, this chapter gives an outlook on an analysis technique that could provide even more precise parameter determinations by considering correlated noise due to stellar variability for example. The chapter concludes with an outlook on possibilities for future follow-up observations.

\subsection{The disappearing transits of Kepler-9c}

With three confirmed planets orbiting Kepler-9, the system is the first multi-planet system detected by the transit method, over and above it is the first system where transit timing variations were measured (Holman et al., 2010). The early detection of this system during the time of the Kepler mission, demonstrated the high impact the telescope will have for measuring transiting exoplanet systems and dynamical interactions between the planets of a system resulting in TTVs. Before, only hot Jupiter type planets in single-planet systems were discovered with the transit detection technique. For these planets no TTV signals are measured due to the lack of further exoplanets in their vicinity.

In this thesis, the characterisation of the Kepler-9 system is brought to a new level by using a photodynamical model to analyse the long and short cadence data from the Kepler telescope of the system accomplished by ground-based follow-up transit observations collected in the framework of the KOINet. The modelled planetary parameters are determined with a precision of about $\sim 1 \%$ by the analysis, this above all also applies for the density ratio of the two planets. The planets have the most precise determined density values of exoplanets in the Neptune-mass regime from this extensive, self-consistent analysis. The largest impact on the achievement of these results was the use of a photodynamical model, instead of modelling the transits in- 
dividually and applying a dynamical analysis to the transit times afterwards, and the use of Kepler short cadence data. Since the dynamical cycle of the system was already very well covered by Kepler data, the addition of the KOINet data only led to small improvements. Nevertheless, this project showed the functionality of the KOINet and its data reduction pipeline. The timing accuracy of KOINet data was investigated by the simultaneous observation of a transit of Kepler-9b with four different telescopes located at different sites. The consistency of the modelled transit times proofed the possibility of using ground-based observations from several telescopes to follow-up one target. Additional to the planetary parameters, the photodynamical analysis models the stellar density. Combining this parameter together with stellar effective temperature, surface gravity and metallicity values derived from high resolution spectroscopic observations by Petigura et al. (2017) in stellar evolution models led to a precise determination of the stellar age, mass and radius. Another advantage of the photodynamical analysis is the self-consistent modelling of all transits simultaneously. This feature led to the possibility of picking up the small transit duration variations in the transits of the Kepler-9b/c system resulting in the prediction of the transits of Kepler-9c to disappear at around 2050. The reason for that is a precession of the planetary orbits due to mutual inclination changes. Likewise, planet b will move towards the lower latitudes of the star in the upcoming years. Therefore, future transit observations of both planets will scan the different latitudes of the star, with planet $\mathrm{c}$ the limb will be measured and planet $\mathrm{b}$ will get into regions where star spots can occur, that means possible spot crossings during transit.

The Kepler-9b/c planets have a period ratio near the 2:1 commensurability, but the resonance angles are oscillating in the systems. This means the planets are not in mean-motion resonance, but close enough to show strong TTVs. It is also a sign for stability as the planet-planet interaction is not that strong to disrupt the system. Nevertheless, the stability was investigated by a numerical integration of the system over 1 Gyr. During this integration the system remained stable.

Recently, Kipping et al. (2019) detected a system in the TESS data similar to Kepler-9b/c with two gaseous, low-density planets in similar distance to its host star. These planets are showing strong anti-correlated, sinusoidal like TTVs as well. Another system of similar architecture is Kepler-46 (Nesvorný et al., 2012, Saad-Olivera et al. 2017), where additional to two gaseous Neptune-like TTV planets a small companion much closer to the host star is found. This planet is similar to Kepler-9d. The system architecture similarities leads to the question of the occurrence rate of such systems and their formation mechanism.

In future photodynamical analysis of the Kepler-9 system, the light curves can be combined with the new radial velocity measurements obtained by Borsato et al. (2019). The authors studied the discrepancy in the mass determination between radial velocity and TTV measurements. Both analysis yield mass determinations consistent to each other and to the results of the photodynamical analysis conducted in this thesis. The combination of transit light curves and radial velocity curves in a photodynamical analysis is already successfully performed on for example Kepler-117 (Almenara et al., 2015), K2-19 (Barros et al., 2015), WASP-47 (Almenara et al., 2016), 
Kepler-419 (Almenara et al., 2018), and Kepler-88 (Weiss et al., 2019). Additionally, the photometric and spectroscopic data of the triple star system EPIC 249432662 (Borkovits et al., 2019a) and the quadruple star system HIP 41431 (Borkovits et al., 2019b) have been photodynamically analysed.

\subsection{A non-transiting planet orbiting Kepler-82}

Four confirmed planets were known from transit observations in the Kepler-82 system. Two smaller inner planets do not show TTV signals and are dynamically independent from the outer two Neptune-size planets that have a period ratio near the 2:1 commensurability and show strong TTV signals. The TTVs of these two larger and outer planets, Kepler-82b/c, were only used before to calculate nominal masses of the planets assuming that one of the planets is solely responsible for the TTVs of the other planet. A dynamical analysis of the TTVs was not examined for this system before.

In this thesis, a photodynamical analysis of the Kepler-82b/c system was carried out on Kepler long and short cadence data combined with new KOINet transit observations. A chopping component in the TTVs of planet c can not be explained by the interaction with planet $b$. Therefore, a third planet was introduced. Based on the chopping pattern with a jump after each three consecutive transits in the transit times, an outer planet with a period ratio to planet c near the $3: 2$ or $3: 1$ commensurability are the two possible solutions. Photodynamical analysis of both planetary systems on Kepler data results in similar probabilities with a small preference for the planet near a 3:2 period ratio to planet c. Shortly after Kepler observations of this object ended, the two models start to differ in its transit time predictions. With the ground-based follow-up observations performed in the framework of the KOINet, the preference of the system model with the unknown planet near a 3:2 period ratio can be confirmed. The resulting planetary system resembles the main peaks from the frequency analysis of the TTVs performed by Ofir et al. (2018) when calculating TTV frequencies based on the TTV super periods and chopping periods. Additionally, the new planet avoids the inclination regime where transit measurements are possible. This is important as the mass of this planet is 1.5 times higher than the one of planet $c$ and, therefore, a larger radius can be assumed which means a higher detectability probability for this planet. The photodynamical analysis of the Kepler-82 system performed in this thesis results therewith in the detection of a fifth planet in the system, called Kepler-82f. Additionally, the determined stellar density together with spectroscopic stellar parameters helped for inferring precise stellar age, mass and radius values in the similar way as for Kepler-9. The planetary densities of planet $\mathrm{b}$ and $\mathrm{c}$ are modelled as well. These values are highly dependent on the assumed planetary system model. When only the two transiting planets are taken into account the density ratio between the two planets acquires a very unusual high value of $\sim 14$. By including the non-transiting planet with a period ratio to planet $\mathrm{c}$ near the 3:2 commensurability, the density ratio of planet $b$ to $c$ reduces to a more common value of $\sim 2$.

Although the Kepler-82 planets b, $\mathrm{c}$ and $\mathrm{f}$ have a period ratio near 1:2:3 commensurability, non of the planet pairs have librating resonance angles, nor is the Laplace 
resonance angle of the three planets librating. The absence of a mean-motion resonance makes the planetary system stable as confirmed by a numerical integration of the system over 10 Gyr.

Similar to Kepler-9b/c the three giant planets in the Kepler-82 are subject to mutual inclination changes. Therefore, the invention of the Kepler telescope only few hundred years earlier or later could have shown another combination of transiting planets.

\subsection{Multi-planet systems}

Both systems analysed in this thesis, Kepler-9 and Kepler-82, are multi-planet systems. Kepler- 9 has three confirmed planets in total and Kepler- 82 got to one of the the 21 detected five-planets systems reported in the NASA Exoplanet archive (2019, September 15) by the examination in this thesis. Therefore, a classification of the results in the current literature on multi-planet systems will be conducted in the following.

Firstly, the systems will be classified in the abundances of distances, radii and masses in comparison with single-planet systems discussed in Figure 1.4. The planets considered during the photodynamical analysis in both systems, Kepler-9b/c and Kepler-82b/c/f, are conform with the observation of planets in multi-planet systems to span a wider range of distances in the outskirts in comparison to planets in singleplanet systems. The inner planets of the systems, Kepler-9d and Kepler-82d/e, fit into the distribution as well. All planets considered in the dynamical analysis are with its radii and masses of Neptune-like nature. Hence, they fall in a valley where only a small fraction of transiting planets are detected in single and in multiple systems, but they are not large enough to fall into the second peak of planets mainly found in single-planet systems with sizes of the order of Jupiter. The detection by Ciardi et al. (2013) of a tendency of the outer planet to be the larger one for planet pairs larger than Neptune is not matched by the Kepler-9b/c system, where Kepler-9b is the more massive planet. In contrast, the three Neptune-size planets Kepler- $82 \mathrm{~b} / \mathrm{c} / \mathrm{d}$ are arranged from close to far by their mass with the inner planet the lightest one. This system is conform with the observation by Ciardi et al. (2013). Additionally, these three planets in the Kepler-82 system are conform with the 'peas in a pod' theory (Weiss et al., 2018, Millholland et al., 2017): they are regular spaced and of similar size. This is another argument against a new planet near the 3:1 period commensurability to planet c, such a planet would have a mass of $\sim 23$ times the mass of planet $c$, instead of $\sim 1.5$ times in case of Kepler-82f. The outer planetary system can be considered independently in this matter, since the two inner planets are far apart from the outer three planets.

The prediction of the disappearing transits for Kepler-9c, as well as the detection of a non-transiting planet, Kepler-82f, present a possibility to explain missing planets in the regular spacing of transiting systems. Here, the planets can appear or disappear due to orbital precession provoked by planet-planet interactions. Precession was also measured in for example the K2-146 system, where Hamann et al. (2019) detected 
the transits of the second planet to appear during the observation period due to a decreasing impact parameter. In this system, the reason for the orbital precession is as well planet-planet interaction. For the many transiting systems with a planet showing TTVs that can not be explained by any other detected planet in the system such precession effects can be the reason for the perturber to be non-transiting. Additionally, some of the gaps in a more or less regular spacing between planets in multi-planet systems (Titius-Bode law, discussions by e.g. Hayes \& Tremaine, 1998, Laskar, 2000) could be filled by planets that are occasionally out of the transiting plane due to the interaction with the other planets. This possibility substantiates the predictions by Sandford et al. (2019) about undetected planets in the Kepler planetary systems. The planet Kepler-82f could not be detected by transits due to slight differences in the inclination in comparison to the other system planets and the same could have been happened to Kepler-9c if the system would have been detected a hundred years later. This is a possible scenario for the other planetary systems detected by Kepler as well.

\subsection{Correlated noise}

Additional to the random white noise, an important type of noise in photometric data is time-correlated noise. The source of correlated noise can be astrophysical and instrumental. Stars can show for example granulation on its surface or other activities that lead to correllated noise. Further examples for correlated noise are telluric variations from changing weather conditions, imperfect flat-fielding, differential extinction or not perfectly modelled telescope systematics. Considering correlated noise in the detrending of stellar light curves rather than just a modelling with a polynomial would lead to improvements of the results of further analysis. While white noise is easily handled in a $\chi^{2}$-minimisation for example, correlated noise needs more sophisticated methods. One of these techniques are Gaussian processes, a stochastical process where every finite collection of variables is a multivariate Gaussian random variable. When computing a likelihood function including Gaussian processes for considering correlated noise the computing time usually scales as the cube of the number of data points. This is an obstacle for usage on large data samples like the Kepler observations. Foreman-Mackey et al. (2017) developed an alternative of Gaussian process computation where the computing time scales linearly with the number of observation points. This is realised by using specific classes of covariances matrices that have a semiseparable structure. With them the system can be directly factorised and solved. This technique is only applicable for one-dimensional data sets. Implemented is this method in the python routine called celerite.

One source of correlated noise in stellar light curves are asteroseismic signals induced by stellar oscillations. Solar-like oscillators are dominated by pressure modes (p-modes). The p-mode frequency pattern, when $\nu_{n, l}$ is the frequency of an oscillation mode with radial order $n$ and spherical degree $l$, can be described by (e.g. Tassoul, 1980, Mosser et al., 2011):

$$
\nu_{n, l} \simeq \Delta \nu\left(n+\frac{l}{2}+\epsilon\right)-\delta \nu_{0, l}
$$




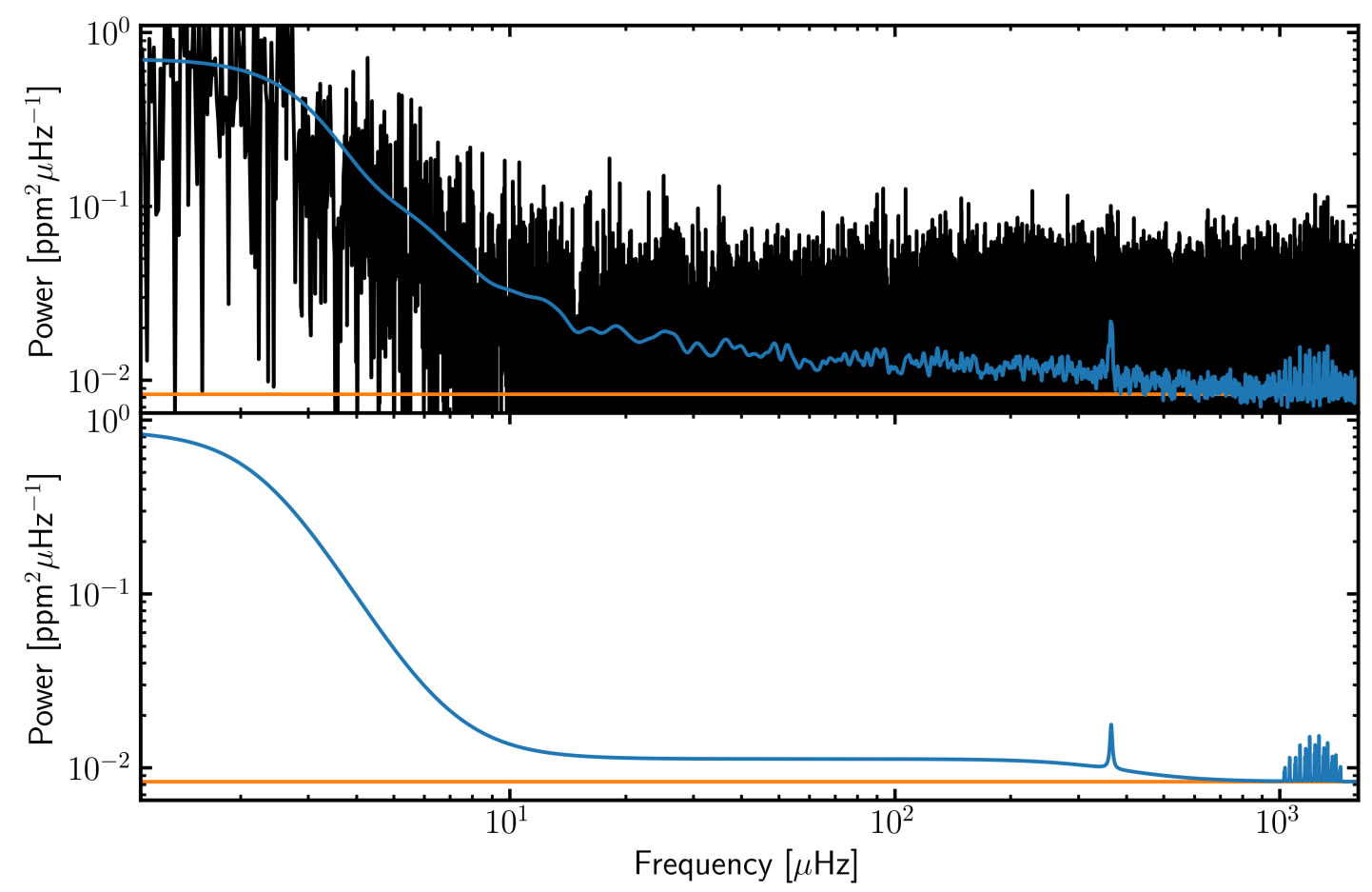

Figure 4.1: Periodogram of the Kepler short cadence data of Kepler-36 without transits. The top shows the Lomb-Scargle spectrum in black and a smoothed version in blue. The orange line indicates the value of the white noise. The bottom plot shows the data modelled with celerite in blue and the white noise value in orange.

with the mean large frequency separation $\delta \nu$, the offset of the radial $l=0$ modes $\epsilon$, and the small frequency separation of non-radial modes $\delta \nu_{0, l}$. Such stellar oscillations are connected to the stellar mass, radius and density. Therefore, a modelling of measured stellar oscillations yields another method for stellar parameter determinations. Such a modelling can be performed with celerite as shown by Foreman-Mackey et al. (2017).

An interesting target that shows asteroseismic signals and TTV planets is the Kepler-36 system (Carter et al., 2012). The two transiting planets are close to each other with a period ratio near the 6:7 commensurability and show strong, anticorrelated TTVs revealing a very high density ratio. A periodogram of the Kepler-36 light curve (like in the top of Figure 4.1 and Figure 4.2) shows asteroseismic signals with frequencies around $\sim 1250 \mu \mathrm{Hz}$ with spherical orders $l=0,1,2$ at a few radial orders, $n$. Carter et al. (2012) performed a classic asteroseismic analysis on the available Kepler short cadence data and used the determined stellar mass and density from this analysis as priors in a photodynamical analysis of the first ten quarters of Kepler data. Combining the asteroseismic analysis directly with the photodynamical model by using celerite and treating the oscillations as correlated noise in the light curve would be the overall goal in order to improve the stellar and planetary parameter determination of this system. An example where Gaussian processes were successfully 


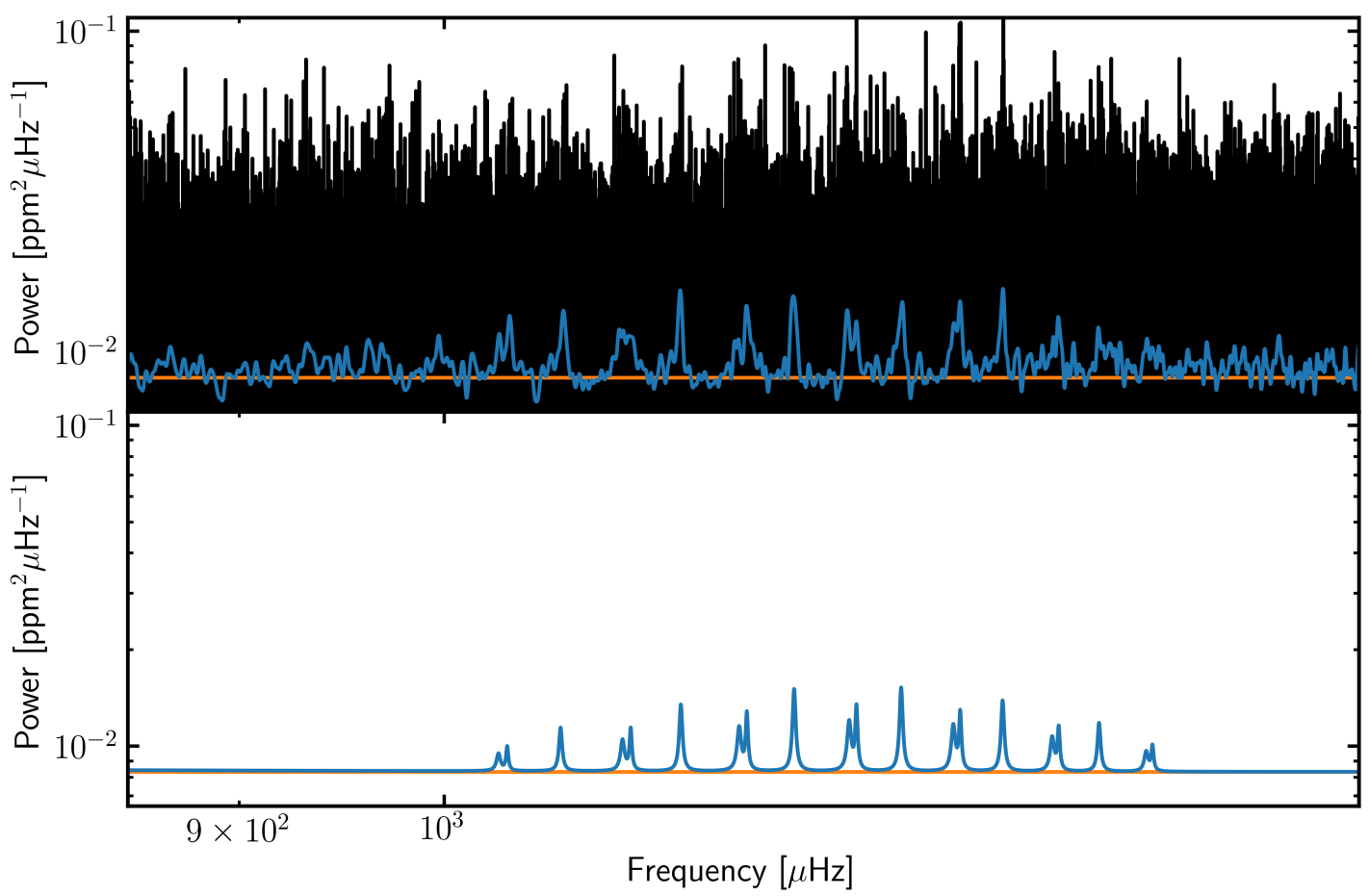

Figure 4.2: Same plot as Figure 4.1, but zoomed into the region with asteroseismic signals.

used for handling the correlated noise due to asteroseismic signals in photometric data is the microlensing event OGLE-2017-BLG-1186, the analysis was performed by Li et al. (2019).

First step in this direction is a correct modelling of the asteroseismic signal with celerite. An initial approach would be the one given in the examples by ForemanMackey et al. (2017). In their example the star only shows oscillations at frequencies of the spherical orders $l=0,2$ and these are so close to each other that they can be modelled as one peak. Therefore, the model needs refinement for the application to Kepler-36. A Lomb-Scargle periodogram (Lomb, 1976, Scargle, 1982, implemented in the astropy package (Astropy Collaboration et al., 2013, 2018) ) of all available Kepler short cadence data with transits cut out (top of Figure 4.1 and Figure 4.2) shows a white noise background, two granulation components (the component with smaller frequencies is related to the super-granulation), a peak at about $365 \mu \mathrm{Hz}$ related to an artefact in the Kepler data and the asteroseismic pattern around $1250 \mu \mathrm{Hz}$. To model the white noise celerite provides a jitter component with the $\sigma$ as free parameter. The quadratic addition of this parameter with the mean uncertainties of the data results in the white noise value. The granulation components can be modelled by a more simple version of the stochastically driven damped simple harmonic oscillator term (SHOTerm) given in equation 24 by (Foreman-Mackey et al., 2017) with the amplitude $S_{g}$ and the frequency $\omega_{g}$ as free parameters. The artefact is modelled by the usual SHOTerm given in equation 20 by (Foreman-Mackey et al., 2017) with an 
amplitude $S_{a}$, width $Q_{a}$ and the frequency $\omega_{a}$ as free parameters. The asteroseismic signals are modelled with the SHOTerm as well, but each peak has its own term and the parameter are not independent from each other. The frequencies are given by the know asteroseismic frequency relation

$$
\begin{aligned}
\omega_{j_{0}, 0} & =2 \pi\left[\nu_{\max }+\left(j_{0}+\epsilon\right) \cdot \Delta \nu\right] \\
\omega_{j_{1}, 1} & =2 \pi\left[\nu_{\max }+\left(j_{1}+\frac{1}{2}+\epsilon\right) \cdot \Delta \nu-\delta_{01}\right] \\
\omega_{j_{2}, 2} & =2 \pi\left[\nu_{\max }+\left(j_{2}+1+\epsilon\right) \cdot \Delta \nu-\delta_{02}\right]
\end{aligned}
$$

and the amplitudes are given by

$$
\begin{aligned}
S_{j_{0}, 0} & =\frac{A_{0}}{Q_{0}^{2}} \exp \left(-\frac{\left[\left(j_{0}+\epsilon\right) \cdot \Delta \nu\right]^{2}}{2 W^{2}}\right) \\
S_{j_{1}, 1} & =\frac{A_{1}}{Q_{1}^{2}} \exp \left(-\frac{\left[\left(j_{1}+\frac{1}{2}+\epsilon\right) \cdot \Delta \nu-\delta_{01}\right]^{2}}{2 W^{2}}\right) \\
S_{j_{2}, 2} & =\frac{A_{2}}{Q_{2}^{2}} \exp \left(-\frac{\left[\left(j_{2}+1+\epsilon\right) \cdot \Delta \nu-\delta_{02}\right]^{2}}{2 W^{2}}\right) .
\end{aligned}
$$

For these terms, $W$ denotes a shared nuisance parameters modelling the width of the whole asteroseismic signal around the maximum frequency $\nu_{\max } \cdot A_{0} A_{1}, A_{2}, Q_{0} Q_{1}$, $Q_{2}$ are nuisance parameter shared between the terms of each spherical order and the radial orders are given by integers running from -3 to 3 for $j_{0}$, from -3 to 2 for $j_{1}$, and from -4 to 2 for $j_{2}$. The asteroseismic parameters mean large frequency separation, $\delta \nu$, the offset of the radial $l=0$ modes, $\epsilon$, and the small frequency separation of non-radial modes, $\delta \nu_{01}$ and $\delta \nu_{02}$ are modelled by this implementation. This model optimised on the Kepler-36 short cadence light curve results in a periodogram given in the bottom of Figure 4.1 and a zoom into the region with the asteroseismic signal is given in Figure 4.1.

This implementation of an asteroseismic pattern does not deliver parameters optimised comparable to classic analysis. Problem is the frequency dependency of $\Delta \nu, \epsilon$, and $\delta \nu_{0, l}$. Possible solutions are to model each peak individually and a calculation of the asteroseismic parameters similar to classic literature (e.g. White et al., 2011) or to implement a weighting of the peaks to account for the frequency dependency similar for example to the work by Mosser et al. (2013). This must be taken into account for comparing stellar parameters resulting from a maximisation of the log-likelihood function given by celerite with the ones from other asteroseismic analysis.

For combining the asteroseismic model with the photodynamical analysis, this idea of the oscillation pattern is good enough when using celerite only for handling the noise of the light curve instead of detrending it by fitting a polynomial to the out-of transit data. This means the asteroseismic parameters would be fixed during an optimisation of the photodynamical model. This is probably necessary anyway to reach a reasonable computing time. The asteroseismic analysis with celerite could be applied afterwards in the refined version described above to the whole light curve with the photodynamical model fixed on the optimised parameters. 


\subsection{Future Observations}

Future follow-up observations of the transits of the discussed planetary systems, Kepler-9b/c and Kepler-82b/c, would improve the system characterisation, especially due to the extended observation baseline. In case of Kepler-9c the observation of further decreasing transit duration would confirm the prediction of the disappearing transits. For the Kepler-82 system the dynamical cycle is not yet fully covered. Resolving the full TTV cycle would serve a more precise planetary parameter characterisation and the confirmation of the system configuration with the non-transiting planet near a 3:2 period ratio to Kepler-82c. Such follow-up observation could be realised from ground, but also from space. The TESS mission (Ricker et al., 2015) launched on 2018 April 18 will visit the Kepler field in one or two sectors. Christ et al. (2019) calculated the probability of the detection of Kepler planets by TESS and the signal-to-noise ratio $(\mathrm{S} / \mathrm{N})$ of their transits. For Kepler-9, they calculated a detection probability of 0.614 (0.954) for planet $\mathrm{b}$, when observed in one sector (two sectors) and for planet $\mathrm{c}$ of $0.286(0.574)$. Hence, there is a very high probability to detect Kepler-9b in the TESS data when observed in two sectors. For Kepler-9c, which would be the object of higher interest due to the possibility of measuring a decrease in the transit duration time, this probability is at least higher than $50 \%$. However, due to the faintness of the host star the expected $\mathrm{S} / \mathrm{N}$ is relatively low with 3.35 (4.80) for Kepler-9b when observed in one sector (two sectors) and 1.95 (3.23) for Kepler-9c. These S/N values can be easily outperformed by ground-based observations, especially when an engineered diffuser is installed at the telescope as it is the case for the 2.5 meter Nordic Optical Telescope (NOT). The S/N of transit observations of Kepler-9b/c with the NOT without the diffuser were already of the order of $\sim 6$ and the diffuser is expected to increase this value a lot (see, von Essen et al., 2019). Kepler-82 is probably too faint for the transits of its planets to be detected by TESS: the planets are not listed by Christ et al. (2019). Another space mission dedicated on observing transiting planets is PLATO (Rauer et al., 2014). Currently, it is planned to be launched in 2026. The capabilities of PLATO to detect the discussed planets are not yet calculated. In conclusion, ground-based follow-up observations are probably the best option to further characterise the planetary systems, especially if telescopes like the NOT with installed diffusers can be accessed. The only disadvantage of ground-based follow-up observations is being tied up to the night-time, which makes the full-coverage of a transit difficult and measuring each transit to resolve the TTV cycle is impossible. The latter is also true for follow-up observations from space as there is currently no mission planned to observe the Kepler field for a longer time. 


\section{Bibliography}

Adams, F. C. \& Laughlin, G. (2003) Migration and dynamical relaxation in crowded systems of giant planets. Icarus, 163(2), 290-306.

Agol, E. \& Deck, K. (2016) Transit Timing to First Order in Eccentricity. ApJ, 818(2), 177.

Agol, E. \& Fabrycky, D. C. (2018) Transit-Timing and Duration Variations for the Discovery and Characterization of Exoplanets. In Handbook of Exoplanets, edited by H. J. Deeg \& J. A. Belmonte, Springer International Publishing, Cham.

Agol, E., Steffen, J., Sari, R. \& Clarkson, W. (2005) On detecting terrestrial planets with timing of giant planet transits. MNRAS, 359, 567-579.

Almenara, J. M., Díaz, R. F., Bonfils, X. \& Udry, S. (2016) Absolute densities, masses, and radii of the WASP-47 system determined dynamically. A\&A, 595, L5.

Almenara, J. M., Díaz, R. F., Hébrard, G., Mardling, R., Damiani, C., Santerne, A., Bouchy, F., Barros, S. C. C. et Al. (2018) SOPHIE velocimetry of Kepler transit candidates. XVIII. Radial velocity confirmation, absolute masses and radii, and origin of the Kepler-419 multiplanetary system. A\&A, 615, A90.

Almenara, J. M., Díaz, R. F., Mardling, R., Barros, S. C. C., Damiani, C., Bruno, G., Bonfils, X. \& Deleuil, M. (2015) Absolute masses and radii determination in multiplanetary systems without stellar models. MNRAS, 453, 2644-2652.

Anglada-Escudé, G., Amado, P. J., Barnes, J., Berdiñas, Z. M., Butler, R. P., Coleman, G. A. L., De La Cueva, I., Dreizler, S. et Al. (2016) A terrestrial planet candidate in a temperate orbit around Proxima Centauri. Nature, 536(7617), 437-440.

Astropy Collaboration, Price-Whelan, A. M., Sipőcz, B. M., Günther, H. M., Lim, P. L., Crawford, S. M., Conseil, S., Shupe, D. L. et al. (2018) The Astropy Project: Building an Open-science Project and Status of the v2.0 Core Package. AJ, 156, 123. 
Astropy Collaboration, Robitaille, T. P., Tollerud, E. J., Greenfield, P., Droettboom, M., Bray, E., Aldcroft, T., Davis, M. et al. (2013) Astropy: A community Python package for astronomy. A\&A, 558, A33.

Barros, S. C. C., Almenara, J. M., Demangeon, O., Tsantaki, M., Santerne, A., Armstrong, D. J., Barrado, D., Brown, D. et Al. (2015) Photodynamical mass determination of the multiplanetary system K2-19. MNRAS, 454, 4267-4276.

Batalha, N. M., Borucki, W. J., Koch, D. G., Bryson, S. T., Haas, M. R., Brown, T. M., Caldwell, D. A., Hall, J. R. et al. (2010) Selection, Prioritization, and Characteristics of Kepler Target Stars. ApJ, 713(2), L109-L114.

Borkovits, T., Rappaport, S., Kaye, T., Isaacson, H., Vanderburg, A., Howard, A. W., Kristiansen, M. H., Omohundro, M. R. et Al. (2019a) Photodynamical analysis of the triply eclipsing hierarchical triple system EPIC 249432662. MNRAS, 483(2), 1934-1951.

Borkovits, T., Sperauskas, J., Tokovinin, A., Latham, D. W., Csányi, I., Hajdu, T. \& Molnár, L. (2019b) The compact multiple system HIP 41431. MNRAS, 487(4), 4631-4647.

Borsato, L., Malavolta, L., Piotto, G., Buchhave, L. A., Mortier, A., Rice, K., Collier Cameron, A., Coffinet, A. et al. (2019) HARPS-N radial velocities confirm the low densities of the Kepler-9 planets. MNRAS, 484(3), 3233-3243.

Borucki, W. J., Koch, D., Basri, G., Batalha, N., Brown, T., Caldwell, D., Caldwell, J., Christensen-Dalsgaard, J. et al. (2010) Kepler PlanetDetection Mission: Introduction and First Results. Science, 327(5968), 977.

Carter, J. A., Agol, E., Chaplin, W. J., Basu, S., Bedding, T. R., Buchhave, L. A., Christensen-Dalsgaard, J., Deck, K. M. et Al. (2012) Kepler-36: A Pair of Planets with Neighboring Orbits and Dissimilar Densities. Science, 337(6094), 556.

Carter, J. A. \& Winn, J. N. (2009) Parameter Estimation from Time-series Data with Correlated Errors: A Wavelet-based Method and its Application to Transit Light Curves. ApJ, 704, 51-67.

CASH, W. (1979) Parameter estimation in astronomy through application of the likelihood ratio. ApJ, 228, 939-947.

Castellano, T., Jenkins, J., Trilling, D. E., Doyle, L. \& Koch, D. (2000) Detection of Planetary Transits of the Star HD 209458 in the Hipparcos Data Set. ApJ, 532(1), L51-L53. 
Chambers, J. E. (1999) A hybrid symplectic integrator that permits close encounters between massive bodies. MNRAS, 304, 793-799.

Christ, C. N., Montet, B. T. \& Fabrycky, D. C. (2019) Observations of the Kepler Field with TESS: Predictions for Planet Yield and Observable Features. AJ, 157(6), 235.

Ciardi, D. R., Fabrycky, D. C., Ford, E. B., Gautier, T. N., I., Howell, S. B., Lissauer, J. J., Ragozzine, D. \& Rowe, J. F. (2013) On the Relative Sizes of Planets within Kepler Multiple-candidate Systems. ApJ, 763(1), 41.

Claret, A. (2000) A new non-linear limb-darkening law for LTE stellar atmosphere models. Calculations for $-5.0<=\log [\mathrm{M} / \mathrm{H}]<=+1,2000 \mathrm{~K}<=\mathrm{T}_{\text {eff }}<=50000 \mathrm{~K}$ at several surface gravities. A\&A, 363, 1081-1190.

Claret, A., Hauschildt, P. H. \& Witte, S. (2013) New limb-darkening coefficients for Phoenix/1d model atmospheres. II. Calculations for $5000 \mathrm{~K} \leqslant \mathrm{~T}_{\text {eff }} \leqslant 10$ 000 K Kepler, CoRot, Spitzer, uvby, UBVRIJHK, Sloan, and 2MASS photometric systems. A\&A, 552, A16.

Deck, K. M. \& Agol, E. (2015) Measurement of Planet Masses with Transit Timing Variations Due to Synodic "Chopping" Effects. ApJ, 802(2), 116.

Deck, K. M. \& Agol, E. (2016) Transit Timing Variations for Planets near Eccentricity-type Mean Motion Resonances. ApJ, 821(2), 96.

Deck, K. M., Agol, E., Holman, M. J. \& Nesvorný, D. (2014) TTVFast: An Efficient and Accurate Code for Transit Timing Inversion Problems. ApJ, 787, 132.

Eastman, J., Siverd, R. \& Gaudi, B. S. (2010) Achieving Better Than 1Minute Accuracy in the Heliocentric and Barycentric Julian Dates. PASP, 122, 935-946.

Ford, E. B., Ragozzine, D., Rowe, J. F., Steffen, J. H., Barclay, T., Batalha, N. M., Borucki, W. J., Bryson, S. T. et Al. (2012) Transit Timing Observations from Kepler. V. Transit Timing Variation Candidates in the First Sixteen Months from Polynomial Models. ApJ, 756(2), 185.

Foreman-Mackey, D., Agol, E., Ambikasaran, S. \& Angus, R. (2017) Fast and Scalable Gaussian Process Modeling with Applications to Astronomical Time Series. AJ, 154(6), 220.

Foreman-Mackey, D., Hogg, D. W., Lang, D. \& Goodman, J. (2013) emcee: The MCMC Hammer. PASP, 125(925), 306.

Freudenthal, J., von Essen, C., Dreizler, S., Wedemeyer, S., Agol, E., Morris, B. M., Becker, A. C., Mallonn, M. ET AL. (2018) Kepler Object of Interest Network. II. Photodynamical modelling of Kepler-9 over 8 years of transit observations. A\&A, 618, A41. 
Freudenthal, J., von Essen, C., Ofir, A., Dreizler, S., Agol, E., Wedemeyer, S., Morris, B. M., Becker, A. C. et Al. (2019) Kepler Object of Interest Network. III. Kepler-82f: a new non-transiting $21 \mathrm{M}_{\oplus}$ planet from photodynamical modelling. A\&A, 628, A108.

Gillon, M., Triaud, A. H. M. J., Demory, B.-O., Jehin, E., Agol, E., Deck, K. M., Lederer, S. M., de Wit, J. et Al. (2017) Seven temperate terrestrial planets around the nearby ultracool dwarf star TRAPPIST-1. Nature, 542(7642), 456-460.

Hamann, A., Montet, B. T., Fabrycky, D. C., Agol, E. \& Kruse, E. (2019) K2-146: Discovery of Planet c, Precise Masses from Transit Timing, and Observed Precession. AJ, 158(3), 133.

Hayes, W. \& Tremaine, S. (1998) Fitting Selected Random Planetary Systems to Titius-Bode Laws. Icarus, 135(2), 549-557.

Holczer, T., Mazeh, T., Nachmani, G., Jontof-Hutter, D., Ford, E. B., Fabrycky, D., Ragozzine, D., Kane, M. et AL. (2016) Transit Timing Observations from Kepler. IX. Catalog of the Full Long-cadence Data Set. ApJS, 225(1), 9.

Holman, M. J., Fabrycky, D. C., Ragozzine, D., Ford, E. B., Steffen, J. H., Welsh, W. F., Lissauer, J. J., Latham, D. W. et al. (2010) Kepler9: A System of Multiple Planets Transiting a Sun-Like Star, Confirmed by Timing Variations. Science, 330(6000), 51.

Holman, M. J. \& Murray, N. W. (2005) The Use of Transit Timing to Detect Terrestrial-Mass Extrasolar Planets. Science, 307(5713), 1288-1291.

Husser, T. O., Wende-von Berg, S., Dreizler, S., Homeier, D., Reiners, A., Barman, T. \& Hauschildt, P. H. (2013) A new extensive library of PHOENIX stellar atmospheres and synthetic spectra. A\&A, 553, A6.

Kane, M., Ragozzine, D., Flowers, X., Holczer, T., Mazeh, T. \& Relles, H. M. (2019) Visual Analysis and Demographics of Kepler Transit Timing Variations. AJ, 157(5), 171.

KidDER, L. E. (1995) Coalescing binary systems of compact objects to (post) $)^{5 / 2}$ Newtonian order. V. Spin effects. Phys. Rev. D, 52, 821-847.

Kipping, D., Nesvorný, D., Hartman, J., Torres, G., Bakos, G., Jansen, T. \& TeAChey, A. (2019) A resonant pair of warm giant planets revealed by TESS. MNRAS, 486(4), 4980-4986.

KIPPING, D. M. (2010) Binning is sinning: morphological light-curve distortions due to finite integration time. MNRAS, 408, 1758-1769. 
KJeldsen, H. \& Frandsen, S. (1992) High-precision time-resolved CCD photometry. PASP, 104, 413-434.

Laskar, J. (2000) On the Spacing of Planetary Systems. Phys. Rev. Lett., 84(15), 3240-3243.

Li, S. S., Zang, W., Udalski, A., Shvartzvald, Y., Huber, D., Lee, C. U., Sumi, T., Gould, A. ET AL. (2019) OGLE-2017-BLG-1186: first application of asteroseismology and Gaussian processes to microlensing. MNRAS, 488(3), 33083323.

Lissauer, J. J., Ragozzine, D., Fabrycky, D. C., Steffen, J. H., Ford, E. B., Jenkins, J. M., Shporer, A., Holman, M. J. et Al. (2011) Architecture and Dynamics of Kepler's Candidate Multiple Transiting Planet Systems. ApJS, 197(1), 8.

Lomb, N. R. (1976) Least-Squares Frequency Analysis of Unequally Spaced Data. Ap\&SS, 39(2), 447-462.

Mandel, K. \& Agol, E. (2002) Analytic Light Curves for Planetary Transit Searches. ApJ, 580, L171-L175.

Mayor, M. \& Queloz, D. (1995) A Jupiter-mass companion to a solar-type star. Nature, 378(6555), 355-359.

Mazeh, T., Nachmani, G., Holczer, T., Fabrycky, D. C., Ford, E. B., Sanchis-Ojeda, R., Sokol, G., Rowe, J. F. ET AL. (2013) Transit Timing Observations from Kepler. VIII. Catalog of Transit Timing Measurements of the First Twelve Quarters. ApJS, 208, 16.

Millholland, S., Wang, S. \& Laughlin, G. (2017) Kepler Multi-planet Systems Exhibit Unexpected Intra-system Uniformity in Mass and Radius. ApJ, 849(2), L33.

MiraldA-Escudé, J. (2002) Orbital Perturbations of Transiting Planets: A Possible Method to Measure Stellar Quadrupoles and to Detect Earth-Mass Planets. ApJ, 564(2), 1019-1023.

Mosser, B., Belkacem, K., Goupil, M. J., Michel, E., Elsworth, Y., Barban, C., Kallinger, T., Hekker, S. ET Al. (2011) The universal redgiant oscillation pattern. An automated determination with CoRoT data. A\&A, 525, L9.

Mosser, B., Michel, E., Belkacem, K., Goupil, M. J., Baglin, A., Barban, C., Provost, J., Samadi, R. et Al. (2013) Asymptotic and measured large frequency separations. A\&A, 550, A126.

Nesvorný, D. \& Beaugé, C. (2010) Fast Inversion Method for Determination of Planetary Parameters from Transit Timing Variations. ApJ, 709(1), L44-L48. 
Nesvorný, D., Kipping, D., Terrell, D., Hartman, J., Bakos, G. Á. \& Buchinave, L. A. (2013) KOI-142, The King of Transit Variations, is a Pair of Planets near the 2:1 Resonance. ApJ, 777(1), 3.

Nesvorný, D., Kipping, D. M., Buchhave, L. A., Bakos, G. Á., Hartman, J. \& Schmitt, A. R. (2012) The Detection and Characterization of a Nontransiting Planet by Transit Timing Variations. Science, 336(6085), 1133.

Nesvorný, D. \& Morbidelli, A. (2008) Mass and Orbit Determination from Transit Timing Variations of Exoplanets. ApJ, 688(1), 636-646.

Nesvorný, D. \& Vokrouhlický, D. (2014) The Effect of Conjunctions on the Transit Timing Variations of Exoplanets. ApJ, 790(1), 58.

Ofir, A., Dreizler, S., Zechmeister, M. \& Husser, T.-O. (2014) An independent planet search in the Kepler dataset. II. An extremely low-density super-Earth mass planet around Kepler-87. A\&A, 561, A103.

Ofir, A., Xie, J.-W., Jiang, C.-F., Sari, R. \& Aharonson, O. (2018) A Spectral Approach to Transit Timing Variations. ApJS, 234(1), 9.

Patil, A., Huard, D. \& Fonnesbeck, C. (2010) PyMC: Bayesian Stochastic Modelling in Python. Journal of Statistical Software, Articles, 35(4), 1-81, URL https://www.jstatsoft.org/v035/i04.

Perryman, M. (2018) The Exoplanet Handbook. Cambridge University Press.

Petigura, E. A., Howard, A. W., Marcy, G. W., Johnson, J. A., IsaAcson, H., Cargile, P. A., Hebb, L., Fulton, B. J. et Al. (2017) The CaliforniaKepler Survey. I. High-resolution Spectroscopy of 1305 Stars Hosting Kepler Transiting Planets. AJ, 154.

Ragozzine, D. \& Holman, M. J. (2010) The Value of Systems with Multiple Transiting Planets. arXiv e-prints, arXiv:1006.3727.

Rauer, H., Catala, C., Aerts, C., Appourchaux, T., Benz, W., BranDeker, A., Christensen-Dalsgaard, J., Deleuil, M. et Al. (2014) The PLATO 2.0 mission. Experimental Astronomy, 38, 249-330.

Ricker, G. R., Winn, J. N., Vanderspek, R., Latham, D. W., Bakos, G. Á., Bean, J. L., Berta-Thompson, Z. K., Brown, T. M. et Al. (2015) Transiting Exoplanet Survey Satellite (TESS). Journal of Astronomical Telescopes, Instruments, and Systems, 1(1), 014003.

Rowe, J. F., Bryson, S. T., Marcy, G. W., Lissauer, J. J., JontofHutter, D., Mullally, F., Gilliland, R. L., Issacson, H. Et Al. (2014) Validation of Kepler's Multiple Planet Candidates. III. Light Curve Analysis and Announcement of Hundreds of New Multi-planet Systems. ApJ, 784(1), 45. 
SaAd-Olivera, X., Nesvorný, D., Kipping, D. M. \& Roig, F. (2017) Masses of Kepler-46b, c from Transit Timing Variations. AJ, 153(4), 198.

Sandford, E., Kipping, D. \& Collins, M. (2019) The multiplicity distribution of Kepler's exoplanets. MNRAS, 489(3), 3162-3173.

SCARGle, J. D. (1982) Studies in astronomical time series analysis. II. Statistical aspects of spectral analysis of unevenly spaced data. ApJ, 263, 835-853.

Soderhjelm, S. (1999) Possible detection of the planet transits of HD 209458 in the Hipparcos photometry. Information Bulletin on Variable Stars, 4816, 1.

Southworth, J., Hinse, T. C., Jørgensen, U. G., Dominik, M., Ricci, D., Burgdorf, M. J., Hornstrup, A., Wheatley, P. J. Et Al. (2009) Highprecision photometry by telescope defocusing - I. The transiting planetary system WASP-5. MNRAS, 396, 1023-1031.

Steele, I. A., Smith, R. J., Rees, P. C., Baker, I. P., Bates, S. D., Bode, M. F., Bowman, M. K., Carter, D. et AL. (2004) The Liverpool Telescope: performance and first results. In Ground-based Telescopes, edited by J. M. Oschmann, Jr., vol. 5489 of Proc. SPIE.

Stefansson, G., Li, Y., Mahadevan, S., Wisniewski, J., HebB, L., MorRis, B., Huehnerhoff, J. \& Hawley, S. (2018a) Diffuser-assisted Photometric Follow-up Observations of the Neptune-sized Planets K2-28b and K2-100b. AJ, 156(6), 266.

Stefansson, G., Mahadevan, S., Hebb, L., Wisniewski, J., Huehnerhoff, J., Morris, B., Halverson, S., Zhao, M. ET Al. (2017) Toward Space-like Photometric Precision from the Ground with Beam-shaping Diffusers. ApJ, 848(1), 9.

Stefansson, G., Mahadevan, S., Wisniewski, J., Li, Y., Hebb, L., MorRis, B., Halverson, S., Monson, A. et AL. (2018b) Extreme precision photometry from the ground with beam-shaping diffusers for K2, TESS, and beyond. In Proc. SPIE, vol. 10702 of Society of Photo-Optical Instrumentation Engineers (SPIE) Conference Series.

Steffen, J. H., Ragozzine, D., Fabrycky, D. C., Carter, J. A., Ford, E. B., Holman, M. J., Rowe, J. F., Welsh, W. F. et Al. (2012) Kepler constraints on planets near hot Jupiters. Proceedings of the National Academy of Science, 109(21), 7982-7987.

TAssoul, M. (1980) Asymptotic approximations for stellar nonradial pulsations. ApJS, 43, 469-490.

Thommes, E. W. \& Lissauer, J. J. (2003) Resonant Inclination Excitation of Migrating Giant Planets. ApJ, 597(1), 566-580. 
von Essen, C., Ofir, A., Dreizler, S., Agol, E., Freudenthal, J., HernánDez, J., Wedemeyer, S., Parkash, V. et Al. (2018) Kepler Object of Interest Network. I. First results combining ground- and space-based observations of Kepler systems with transit timing variations. A\&A, 615, A79.

von Essen, C., Schröter, S., Agol, E. \& Schmitt, J. H. M. M. (2013) Qatar-1: indications for possible transit timing variations. A\&A, 555, A92.

von Essen, C., Stefansson, G., Mallonn, M., Pursimo, T., Duupvik, A. A., Mahadevan, S., Kueldsen, H., Freudenthal, J. et al. (2019) First light of engineered diffusers at the Nordic Optical Telescope reveal time variability of the optical eclipse depth of WASP-12b. A\&A, 628, A115.

Weiss, L. M., Agol, E., Fabrycky, D. C., Mills, S. M., Howard, A. W., Isaacson, H., Petigura, E. A., Fulton, B. J. Et Al. (2019) The Discovery of the Long-Period, Eccentric Planet Kepler-88 d and System Characterization with Radial Velocities and Photodynamical Analysis. arXiv e-prints, arXiv:1909.02427.

Weiss, L. M., Marcy, G. W., Petigura, E. A., Fulton, B. J., Howard, A. W., Winn, J. N., Isaacson, H. T., Morton, T. D. et Al. (2018) The California-Kepler Survey. V. Peas in a Pod: Planets in a Kepler Multi-planet System Are Similar in Size and Regularly Spaced. AJ, 155(1), 48.

White, T. R., Bedding, T. R., Stello, D., Christensen-Dalsgaard, J., Huber, D. \& KJeldsen, H. (2011) Calculating Asteroseismic Diagrams for Solarlike Oscillations. ApJ, 743(2), 161.

XIE, J.-W . (2013) Transit Timing Variation of Near-resonance Planetary Pairs: Confirmation of 12 Multiple-planet Systems. ApJS, 208(2), 22.

XIE, J.-W. (2014) Transit Timing Variation of Near-resonance Planetary Pairs. II. Confirmation of 30 Planets in 15 Multiple-planet Systems. ApJS, 210(2), 25.

Zechmeister, M., Dreizler, S., Ribas, I., Reiners, A., Caballero, J. A., Bauer, F. F., BéJar, V. J. S., González-Cuesta, L. et Al. (2019) The CARMENES search for exoplanets around M dwarfs. Two temperate Earth-mass planet candidates around Teegarden's Star. A\&A, 627, A49.

Zipf, G. K. (1935) The psycho-biology of language. Houghton, Mifflin, Boston. 


\section{Acknowledgements}

First of all I would like to thank Stefan Dreizler. For great support already since my bachelor thesis, the unshakeable faith in my abilities, the open ear at any time, thank you! Furthermore, I thank Caro for her positivity, her enthusiasm and her support. I also thank Rick for fruitful discussions and the open door at all times. And I thank Eric Agol for the scientific exchange and eventful days in Seattle. I thank the working group for helpful discussions in the group meetings and beyond, as well as joyful meetings after work.

During my studies I got to know a lot of great people, whom I thank for all the hours together. From the very beginning Alina, Natalie, Birte, René, Björn, Stefan and Adrian were with me. I owe you the fact that I survived the first semesters and of course we spent a lot of happy times during our studies and afterwards. Later Bekki, Benjamin and Philipp joined, thank you for your friendship. Sharing the office with Benjamin resulted in fruitful discussions about the work and also the one or other distraction with other topics.

I would like to thank my family, especially my parents, for their loving support throughout my studies, without you it would have been much more difficult. It is always nice to come back home. Last but not least I thank Martin for love and comfort, you are always there for me.

This project was funded by the German Research Foundation (DFG) through grant DR 281/30-1. 
